DESY-08-175

December 2008

\title{
Deep inelastic scattering with leading protons or large rapidity gaps at HERA
}

\author{
ZEUS Collaboration
}

\begin{abstract}
The dissociation of virtual photons, $\gamma^{\star} p \rightarrow X p$, in events with a large rapidity gap between $X$ and the outgoing proton, as well as in events in which the leading proton was directly measured, has been studied with the ZEUS detector at HERA. The data cover photon virtualities $Q^{2}>2 \mathrm{GeV}^{2}$ and $\gamma^{\star} p$ centre-of-mass energies $40<W<240 \mathrm{GeV}$, with $M_{X}>2 \mathrm{GeV}$, where $M_{X}$ is the mass of the hadronic final state, $X$. Leading protons were detected in the ZEUS leading proton spectrometer. The cross section is presented as a function of $t$, the squared four-momentum transfer at the proton vertex and $\Phi$, the azimuthal angle between the positron scattering plane and the proton scattering plane. It is also shown as a function of $Q^{2}$ and $x_{\mathbb{P}}$, the fraction of the proton's momentum carried by the diffractive exchange, as well as $\beta$, the Bjorken variable defined with respect to the diffractive exchange.
\end{abstract}




\section{The ZEUS Collaboration}

S. Chekanov, M. Derrick, S. Magill, B. Musgrave, D. Nicholass ${ }^{1}$, J. Repond, R. Yoshida Argonne National Laboratory, Argonne, Illinois 60439-4815, USA ${ }^{n}$

M.C.K. Mattingly

Andrews University, Berrien Springs, Michigan 49104-0380, USA

P. Antonioli, G. Bari, L. Bellagamba, D. Boscherini, A. Bruni, G. Bruni, G. Cara Romeo

F. Cindolo, M. Corradi, G. Iacobucci, A. Margotti, T. Massam, R. Nania, A. Polini INFN Bologna, Bologna, Italy ${ }^{e}$

S. Antonelli, M. Basile, M. Bindi, L. Cifarelli, A. Contin, F. Palmonari, S. De Pasquale², G. Sartorelli, A. Zichichi

University and INFN Bologna, Bologna, Italy ${ }^{e}$

D. Bartsch, I. Brock, H. Hartmann, E. Hilger, H.-P. Jakob, M. Jüngst, A.E. Nuncio-Quiroz,

E. Paul, U. Samson, V. Schönberg, R. Shehzadi, M. Wlasenko

Physikalisches Institut der Universität Bonn, Bonn, Germany ${ }^{b}$

N.H. Brook, G.P. Heath, J.D. Morris

H.H. Wills Physics Laboratory, University of Bristol, Bristol, United Kingdom ${ }^{m}$

M. Kaur, P. Kaur ${ }^{3}$, I. Singh ${ }^{3}$

Panjab University, Department of Physics, Chandigarh, India

M. Capua, S. Fazio, A. Mastroberardino, M. Schioppa, G. Susinno, E. Tassi

Calabria University, Physics Department and INFN, Cosenza, Italy ${ }^{e}$

J.Y. Kim

Chonnam National University, Kwangju, South Korea

Z.A. Ibrahim, F. Mohamad Idris, B. Kamaluddin, W.A.T. Wan Abdullah

Jabatan Fizik, Universiti Malaya, 50603 Kuala Lumpur, Malaysia ${ }^{r}$

Y. Ning, Z. Ren, F. Sciulli

Nevis Laboratories, Columbia University, Irvington on Hudson, New York 10027º

J. Chwastowski, A. Eskreys, J. Figiel, A. Galas, K. Olkiewicz, B. Pawlik, P. Stopa,

L. Zawiejski

The Henryk Niewodniczanski Institute of Nuclear Physics, Polish Academy of Sciences, Cracow, Poland ${ }^{i}$

L. Adamczyk, T. Bołd, I. Grabowska-Bołd, D. Kisielewska, J. Łukasik ${ }^{4}$, M. Przybycień,

L. Suszycki

Faculty of Physics and Applied Computer Science, AGH-University of Science and Technology, Cracow, Poland ${ }^{p}$ 
A. Kotański ${ }^{5}$, W. Słomiński ${ }^{6}$

Department of Physics, Jagellonian University, Cracow, Poland

O. Behnke, U. Behrens, C. Blohm, A. Bonato, K. Borras, D. Bot, R. Ciesielski, N. Coppola, S. Fang, J. Fourletova ${ }^{7}$, A. Geiser, P. Göttlicher ${ }^{8}$, J. Grebenyuk, I. Gregor, T. Haas, W. Hain, A. Hüttmann, F. Januschek, B. Kahle, I.I. Katkov ${ }^{9}$, U. Klein ${ }^{10}$, U. Kötz, H. Kowalski, M. Lisovyi, E. Lobodzinska, B. Löhr, R. Mankel ${ }^{11}$, I.-A. Melzer-Pellmann, S. Miglioranzi ${ }^{12}$, A. Montanari, T. Namsoo, D. Notz ${ }^{11}$, A. Parenti, L. Rinaldi ${ }^{13}$, P. Roloff, I. Rubinsky, U. Schneekloth, A. Spiridonov ${ }^{14}$, D. Szuba ${ }^{15}$, J. Szuba ${ }^{16}$, T. Theedt, J. Ukleja ${ }^{17}$, G. Wolf, K. Wrona, A.G. Yagües Molina, C. Youngman, W. Zeuner ${ }^{11}$

Deutsches Elektronen-Synchrotron DESY, Hamburg, Germany

V. Drugakov, W. Lohmann, S. Schlenstedt

Deutsches Elektronen-Synchrotron DESY, Zeuthen, Germany

G. Barbagli, E. Gallo

INFN Florence, Florence, Italy ${ }^{e}$

P. G. Pelfer

University and INFN Florence, Florence, Italy $^{e}$

A. Bamberger, D. Dobur, F. Karstens, N.N. Vlasov ${ }^{18}$

Fakultät für Physik der Universität Freiburg i.Br., Freiburg i.Br., Germany ${ }^{b}$

P.J. Bussey ${ }^{19}$, A.T. Doyle, W. Dunne, M. Forrest, M. Rosin, D.H. Saxon, I.O. Skillicorn Department of Physics and Astronomy, University of Glasgow, Glasgow, United Kingdom ${ }^{m}$

I. Gialas $^{20}$, K. Papageorgiu

Department of Engineering in Management and Finance, Univ. of Aegean, Greece

U. Holm, R. Klanner, E. Lohrmann, H. Perrey, P. Schleper, T. Schörner-Sadenius, J. Sztuk, H. Stadie, M. Turcato

Hamburg University, Institute of Exp. Physics, Hamburg, Germany ${ }^{b}$

C. Foudas, C. Fry, K.R. Long, A.D. Tapper

Imperial College London, High Energy Nuclear Physics Group, London, United Kingdom m

T. Matsumoto, K. Nagano, K. Tokushuku ${ }^{21}$, S. Yamada, Y. Yamazaki22

Institute of Particle and Nuclear Studies, KEK, Tsukuba, Japan ${ }^{f}$

A.N. Barakbaev, E.G. Boos, N.S. Pokrovskiy, B.O. Zhautykov

Institute of Physics and Technology of Ministry of Education and Science of Kazakhstan, Almaty, Kazakhstan 
V. Aushev ${ }^{23}$, O. Bachynska, M. Borodin, I. Kadenko, A. Kozulia, V. Libov, D. Lontkovskyi, I. Makarenko, Iu. Sorokin, A. Verbytskyi, O. Volynets

Institute for Nuclear Research, National Academy of Sciences, Kiev and Kiev National University, Kiev, Ukraine

D. Son

Kyungpook National University, Center for High Energy Physics, Daegu, South Korea ${ }^{g}$

J. de Favereau, K. Piotrzkowski

Institut de Physique Nucléaire, Université Catholique de Louvain, Louvain-la-Neuve, Belgium ${ }^{q}$

F. Barreiro, C. Glasman, M. Jimenez, L. Labarga, J. del Peso, E. Ron, M. Soares,

J. Terrón, C. Uribe-Estrada, M. Zambrana

Departamento de Fúsica Teórica, Universidad Autónoma de Madrid, Madrid, Spain ${ }^{l}$

F. Corriveau, C. Liu, J. Schwartz, R. Walsh, C. Zhou

Department of Physics, McGill University, Montréal, Québec, Canada H3A 2T8 ${ }^{a}$

T. Tsurugai

Meiji Gakuin University, Faculty of General Education, Yokohama, Japan ${ }^{f}$

A. Antonov, B.A. Dolgoshein, D. Gladkov, V. Sosnovtsev, A. Stifutkin, S. Suchkov Moscow Engineering Physics Institute, Moscow, Russia ${ }^{j}$

R.K. Dementiev, P.F. Ermolov ${ }^{\dagger}$, L.K. Gladilin, Yu.A. Golubkov, L.A. Khein, I.A. Korzhavina, V.A. Kuzmin, B.B. Levchenko ${ }^{24}$, O.Yu. Lukina, A.S. Proskuryakov, L.M. Shcheglova, D.S. Zotkin

Moscow State University, Institute of Nuclear Physics, Moscow, Russia ${ }^{k}$

I. Abt, A. Caldwell, D. Kollar, B. Reisert, W.B. Schmidke

Max-Planck-Institut für Physik, München, Germany

G. Grigorescu, A. Keramidas, E. Koffeman, P. Kooijman, A. Pellegrino, H. Tiecke, M. Vázquez ${ }^{12}$, L. Wiggers

NIKHEF and University of Amsterdam, Amsterdam, Netherlands ${ }^{h}$

N. Brümmer, B. Bylsma, L.S. Durkin, A. Lee, T.Y. Ling

Physics Department, Ohio State University, Columbus, Ohio $43210^{n}$

P.D. Allfrey, M.A. Bell, A.M. Cooper-Sarkar, R.C.E. Devenish, J. Ferrando, B. Foster,

C. Gwenlan ${ }^{25}$, K. Horton ${ }^{26}$, K. Oliver, A. Robertson, R. Walczak

Department of Physics, University of Oxford, Oxford United Kingdom ${ }^{m}$

A. Bertolin, F. Dal Corso, S. Dusini, A. Longhin, L. Stanco

INFN Padova, Padova, Italy ${ }^{e}$ 
P. Bellan, R. Brugnera, R. Carlin, A. Garfagnini, S. Limentani

Dipartimento di Fisica dell' Università and INFN, Padova, Italy ${ }^{e}$

B.Y. Oh, A. Raval, J.J. Whitmore ${ }^{27}$

Department of Physics, Pennsylvania State University, University Park, Pennsylvania $16802^{\circ}$

Y. Iga

Polytechnic University, Sagamihara, Japan ${ }^{f}$

G. D'Agostini, G. Marini, A. Nigro

Dipartimento di Fisica, Università 'La Sapienza' and INFN, Rome, Italy ${ }^{e}$

J.E. Cole ${ }^{28}$, J.C. Hart

Rutherford Appleton Laboratory, Chilton, Didcot, Oxon, United Kingdom ${ }^{m}$

C. Heusch, H. Sadrozinski, A. Seiden, R. Wichmann ${ }^{29}$, D.C. Williams

University of California, Santa Cruz, California 95064, USA ${ }^{n}$

H. Abramowicz ${ }^{30}$, R. Ingbir, S. Kananov, A. Levy, A. Stern

Raymond and Beverly Sackler Faculty of Exact Sciences, School of Physics, Tel Aviv University, Tel Aviv, Israel ${ }^{d}$

M. Kuze, J. Maeda

Department of Physics, Tokyo Institute of Technology, Tokyo, Japan ${ }^{f}$

R. Hori, S. Kagawa ${ }^{31}$, N. Okazaki, S. Shimizu, T. Tawara

Department of Physics, University of Tokyo, Tokyo, Japan ${ }^{f}$

R. Hamatsu, H. Kaji ${ }^{32}$, S. Kitamura ${ }^{33}$, O. Ota ${ }^{34}$, Y.D. Ri

Tokyo Metropolitan University, Department of Physics, Tokyo, Japan ${ }^{f}$

R. Cirio, M. Costa, M.I. Ferrero, V. Monaco, C. Peroni, R. Sacchi, V. Sola, A. Solano

Università di Torino and INFN, Torino, Italy ${ }^{e}$

N. Cartiglia, S. Maselli, A. Staiano

INFN Torino, Torino, Italy ${ }^{e}$

M. Arneodo, M. Ruspa

Università del Piemonte Orientale, Novara, and INFN, Torino, Italy ${ }^{e}$

S. Fourletov ${ }^{7}$, J.F. Martin, T.P. Stewart

Department of Physics, University of Toronto, Toronto, Ontario, Canada M5S 1A7 a

S.K. Boutle ${ }^{20}$, J.M. Butterworth, T.W. Jones, J.H. Loizides, M. Wing ${ }^{35}$

Physics and Astronomy Department, University College London, London, United Kingdom ${ }^{m}$ 
B. Brzozowska, J. Ciborowski ${ }^{36}$, G. Grzelak, P. Kulinski, P. Łużniak ${ }^{37}$, J. Malka ${ }^{37}$, R.J. Nowak, J.M. Pawlak, W. Perlanski ${ }^{37}$, T. Tymieniecka ${ }^{38}$, A.F. Żarnecki

Warsaw University, Institute of Experimental Physics, Warsaw, Poland

M. Adamus, P. Plucinski ${ }^{39}$, A. Ukleja

Institute for Nuclear Studies, Warsaw, Poland

Y. Eisenberg, D. Hochman, U. Karshon

Department of Particle Physics, Weizmann Institute, Rehovot, Israel ${ }^{c}$

E. Brownson, D.D. Reeder, A.A. Savin, W.H. Smith, H. Wolfe

Department of Physics, University of Wisconsin, Madison, Wisconsin 53706, USA ${ }^{n}$

S. Bhadra, C.D. Catterall, Y. Cui, G. Hartner, S. Menary, U. Noor, J. Standage, J. Whyte

Department of Physics, York University, Ontario, Canada MзJ 1Pз ${ }^{a}$ 
1 also affiliated with University College London, United Kingdom

2 now at University of Salerno, Italy

${ }^{3}$ also working at Max Planck Institute, Munich, Germany

${ }^{4}$ now at Institute of Aviation, Warsaw, Poland

${ }^{5}$ supported by the research grant no. 1 P03B 04529 (2005-2008)

6 This work was supported in part by the Marie Curie Actions Transfer of Knowledge project COCOS (contract MTKD-CT-2004-517186)

${ }^{7}$ now at University of Bonn, Germany

${ }^{8}$ now at DESY, group FEB, Hamburg, Germany

${ }^{9}$ also at Moscow State University, Russia

10 now at University of Liverpool, UK

11 on leave of absence at CERN, Geneva, Switzerland

12 now at CERN, Geneva, Switzerland

13 now at Bologna University, Bologna, Italy

14 also at Institut of Theoretical and Experimental Physics, Moscow, Russia

15 also at INP, Cracow, Poland

${ }^{16}$ also at FPACS, AGH-UST, Cracow, Poland

17 partially supported by Warsaw University, Poland

18 partly supported by Moscow State University, Russia

${ }^{19}$ Royal Society of Edinburgh, Scottish Executive Support Research Fellow

${ }^{20}$ also affiliated with DESY, Germany

21 also at University of Tokyo, Japan

22 now at Kobe University, Japan

${ }^{23}$ supported by DESY, Germany

${ }^{24}$ partly supported by Russian Foundation for Basic Research grant no. 05-02-39028NSFC-a

${ }^{25}$ STFC Advanced Fellow

${ }^{26}$ nee Korcsak-Gorzo

27 This material was based on work supported by the National Science Foundation, while working at the Foundation.

${ }^{28}$ now at University of Kansas, Lawrence, USA

${ }^{29}$ now at DESY, group MPY, Hamburg, Germany

30 also at Max Planck Institute, Munich, Germany, Alexander von Humboldt Research Award

${ }^{31}$ now at KEK, Tsukuba, Japan

32 now at Nagoya University, Japan

33 member of Department of Radiological Science, Tokyo Metropolitan University, Japan

${ }^{34}$ now at SunMelx Co. Ltd., Tokyo, Japan

35 also at Hamburg University, Inst. of Exp. Physics, Alexander von Humboldt Research Award and partially supported by DESY, Hamburg, Germany

36 also at Łódź University, Poland

37 member of Lódź University, Poland

38 also at University of Podlasie, Siedlce, Poland

39 now at Lund Universtiy, Lund, Sweden

${ }^{\dagger}$ deceased 
a supported by the Natural Sciences and Engineering Research Council of Canada (NSERC)

$b$ supported by the German Federal Ministry for Education and Research (BMBF), under contract numbers 05 HZ6PDA, 05 HZ6GUA, 05 HZ6VFA and $05 \mathrm{HZ} 4 \mathrm{KHA}$

c supported in part by the MINERVA Gesellschaft für Forschung GmbH, the Israel Science Foundation (grant no. 293/02-11.2) and the U.S.-Israel Binational Science Foundation

$d$ supported by the Israel Science Foundation

$e$ supported by the Italian National Institute for Nuclear Physics (INFN)

$f$ supported by the Japanese Ministry of Education, Culture, Sports, Science and Technology (MEXT) and its grants for Scientific Research

$g$ supported by the Korean Ministry of Education and Korea Science and Engineering Foundation

$h \quad$ supported by the Netherlands Foundation for Research on Matter (FOM)

$i$ supported by the Polish State Committee for Scientific Research, project no. DESY/256/2006 - 154/DES/2006/03

$j$ partially supported by the German Federal Ministry for Education and Research $(\mathrm{BMBF})$

$k$ supported by RF Presidential grant N 1456.2008.2 for the leading scientific schools and by the Russian Ministry of Education and Science through its grant for Scientific Research on High Energy Physics

$l$ supported by the Spanish Ministry of Education and Science through funds provided by CICYT

$m$ supported by the Science and Technology Facilities Council, UK

$n$ supported by the US Department of Energy

$o$ supported by the US National Science Foundation. Any opinion, findings and conclusions or recommendations expressed in this material are those of the authors and do not necessarily reflect the views of the National Science Foundation.

$p$ supported by the Polish Ministry of Science and Higher Education as a scientific project (2006-2008)

$q$ supported by FNRS and its associated funds (IISN and FRIA) and by an Inter-University Attraction Poles Programme subsidised by the Belgian Federal Science Policy Office

$r$ supported by an FRGS grant from the Malaysian government 


\section{Introduction}

In diffractive hadron-hadron or photon-hadron collisions, the initial-state particles undergo a "peripheral" collision, in which they either stay intact (elastic scattering), or dissociate into low-mass states (diffractive dissociation). The scattered hadron (or the low-mass state in the dissociative case) has energy equal, to within a few per cent, to that of the incoming hadron, and very small transverse momentum. Such interactions can be described in the framework of Regge phenomenology, where they are ascribed to the exchange of a trajectory with the vacuum quantum numbers, the Pomeron trajectory [1]. In the same framework, events in which the hadron loses a somewhat higher fraction of its energy are ascribed to the exchange of Reggeon and pion trajectories.

Significant progress has been made in understanding diffraction in terms of perturbative Quantum Chromodynamics (pQCD) by studying the dissociation of virtual photons, $\gamma^{\star} p \rightarrow X p$, in diffractive deep inelastic ep scattering (DIS) at HERA, ep $\rightarrow e X p$. The part of the DIS cross section due to such processes may be expressed in terms of the diffractive parton distribution functions (PDFs) of the proton. Diffractive PDFs are defined as the proton PDFs probed when the proton emerges intact from the hard interaction, suffering only a small energy loss.

At high centre-of-mass energy, diffractive $e p$ scattering is characterised by the presence of a leading proton in the final state carrying most of the proton beam energy and by the presence of a large rapidity gap (LRG) in the forward (proton) direction. Both of these signatures have been exploited at the HERA collider to select samples enriched in diffractive events. Alternatively, a method has been used to determine statistically the number of diffractive events, based on the expected difference in shape of the distributions of the invariant mass, $M_{X}$, for diffractive and non-diffractive events. These approaches are subject to different systematic uncertainties.

This paper presents results based on the detection of a leading proton or of a large rapidity gap. The same data have also been analysed in terms of the shape of the $M_{X}$ distribution [2]. For the proton-tagged sample, the ZEUS leading proton spectrometer (LPS) was used; this data sample has events with scattered protons carrying a fraction, $x_{L}$, of at least $90 \%$ of the incoming proton momentum. For $x_{L} \lesssim 0.98-0.99$, the sample is dominated by non-diffractive events, whilst for $x_{L} \approx 1$, it consists almost exclusively of diffractive events; therefore the transition between non-diffractive and diffractive regions is studied. In the LRG sample the proton momentum is not measured, but events are selected on the basis of the variable $x_{\mathbb{P}}$, which is the fraction of the proton's momentum carried by the diffractive exchange, $x_{\mathbb{P}} \simeq 1-x_{L}$. Events in the LRG sample are required to have $x_{\mathbb{P}}<0.02$ and thus the sample mainly ( $\gtrsim 90 \%$ ) consists of diffractive events [3]. The kinematic regions covered by the LRG and LPS results are: photon virtualities 
$2<Q^{2}<305 \mathrm{GeV}^{2}$ (LRG) or $2<Q^{2}<120 \mathrm{GeV}^{2}$ (LPS), photon-proton centre-ofmass energies $40<W<240 \mathrm{GeV}$, hadronic final-state masses $2<M_{X}<25 \mathrm{GeV}$ (LRG) or $2<M_{X}<40 \mathrm{GeV}$ (LPS), proton fractional momentum losses $0.0002<x_{\mathbb{P}}<0.02$ (LRG) or $0.0002<x_{\mathbb{P}}<0.1$ (LPS) and values of the square of the four-momentum exchanged at the proton vertex $0.09<|t|<0.55 \mathrm{GeV}^{2}$ (LPS).

\section{Experimental set-up}

The data used for this measurement were taken with the ZEUS detector at the HERA $e p$ collider in the years 1999 and 2000, when HERA collided positrons of $27.5 \mathrm{GeV}$ with protons of $920 \mathrm{GeV}$. The data used for the LRG and LPS analyses correspond to integrated luminosities of $62.2 \mathrm{pb}^{-1}$ and $32.6 \mathrm{pb}^{-1}$, respectively.

A detailed description of the ZEUS detector can be found elsewhere $[4,5]$. A brief outline of the components that are most relevant for this analysis is given below.

Deep inelastic scattering events were identified using information from the central tracking detector (CTD), the uranium-scintillator calorimeter (CAL), the small angle rear tracking detector (SRTD), the rear part of the hadron-electron separator (RHES) and the forward plug calorimeter (FPC).

Charged particles were tracked in the CTD [6]. The CTD operated in a magnetic field of $1.43 \mathrm{~T}$ provided by a thin solenoid. It consisted of 72 cylindrical drift chamber layers, organised in nine superlayers covering the polar-angle ${ }^{1}$ region $15^{\circ}<\theta<164^{\circ}$. The transverse-momentum resolution for full-length tracks was $\sigma\left(p_{T}\right) / p_{T}=0.0058 p_{T} \oplus 0.0065 \oplus$ $0.0014 / p_{T}$, with $p_{T}$ in $\mathrm{GeV}$.

The CAL [7] consisted of three parts: the forward (FCAL), the barrel (BCAL) and the rear (RCAL) calorimeters. Each part was subdivided transversely into towers and longitudinally into one electromagnetic section (EMC) and either one (in RCAL) or two (in BCAL and FCAL) hadronic sections (HAC). The smallest subdivision of the calorimeter was called a cell. The CAL energy resolutions, as measured under test-beam conditions, were $\sigma(E) / E=0.18 / \sqrt{E}$ for electrons and $\sigma(E) / E=0.35 / \sqrt{E}$ for hadrons, with $E$ in $\mathrm{GeV}$.

The position of electrons scattered at small angles to the electron-beam direction was determined by means of the information from the CAL and the SRTD $[8,9]$. The SRTD was attached to the front face of the RCAL and consisted of two planes of scintillator

\footnotetext{
${ }^{1}$ The ZEUS coordinate system is a right-handed Cartesian system, with the $Z$ axis pointing in the proton direction, referred to as the "forward direction", and the $X$ axis pointing left towards the centre of HERA. The coordinate origin is at the nominal interaction point.
} 
strips, $1 \mathrm{~cm}$ wide and $0.5 \mathrm{~cm}$ thick, arranged in orthogonal orientations. Ambiguities in SRTD hits were resolved with the help of the RHES [10], which consisted of a layer of approximately $10,000\left(2.96 \times 3.32 \mathrm{~cm}^{2}\right)$ silicon-pad detectors inserted in the RCAL at a depth of 3.3 radiation lengths.

The FPC [11] was used to measure the energy of particles in the pseudorapidity range $\eta \approx 4.0-5.0$. It was a lead-scintillator sandwich calorimeter read out by wavelengthshifter (WLS) fibers and photomultipliers (PMT). It was installed in the $20 \times 20 \mathrm{~cm}^{2}$ beam hole of the FCAL. The FPC had outer dimensions of $19.2 \times 19.2 \times 108 \mathrm{~cm}^{3}$ and had a central hole of $3.15 \mathrm{~cm}$ radius to accommodate the beam-pipe. In the FPC, $15 \mathrm{~mm}$ thick lead plates alternated with $2.6 \mathrm{~mm}$ thick scintillator layers. The FPC was subdivided longitudinally into an electromagnetic (10 layers) and a hadronic section (50 layers) representing a total of 5.4 nuclear absorption lengths. The energy resolution for electrons, as measured in a test beam, was $\sigma(E) / E=(0.41 \pm 0.02) / \sqrt{E} \oplus 0.062 \pm 0.002$, with $E$ in $\mathrm{GeV}$. When installed in the FCAL, the energy resolution for pions was $\sigma(E) / E=$ $(0.65 \pm 0.02) / \sqrt{E} \oplus 0.06 \pm 0.01$, with $E$ in $\mathrm{GeV}$, and the $e / h$ ratio was close to unity.

The LPS [12] detected positively charged particles scattered at very small angles and carrying a substantial fraction, $x_{L}$, of the incoming proton momentum; these particles remained in the beam-pipe and their trajectories were measured by a system of silicon microstrip detectors that could be inserted very close (typically a few $\mathrm{mm}$ ) to the proton beam. The detectors were grouped in six stations, S1 to S6, placed along the beam line in the direction of the proton beam, between $23.8 \mathrm{~m}$ and $90.0 \mathrm{~m}$ from the interaction point. The particle deflections induced by the magnets of the proton beam line allowed a momentum analysis of the scattered protons. Only stations S4, S5 and S6 covered the kinematic region of the present measurement. The resolutions were about $0.5 \%$ on the longitudinal momentum fraction and about $5 \mathrm{MeV}$ on the transverse momentum. The effective transverse-momentum resolution was dominated by the intrinsic transversemomentum spread of the proton beam at the interaction point, which was about $45 \mathrm{MeV}$ in the horizontal plane and about $100 \mathrm{MeV}$ in the vertical plane. The LPS acceptance was approximately $2 \%$ and $x_{L}$ independent for $x_{L} \gtrsim 0.98$; it increased smoothly to about $10 \%$ as $x_{L}$ decreased to 0.9 .

The luminosity was determined from the rate of the bremsstrahlung process ep $\rightarrow e \gamma p$. The photon was measured in a lead-scintillator calorimeter [13] placed in the HERA tunnel at $Z=-107 \mathrm{~m}$. 


\section{Kinematics and cross sections}

Figure 1 shows a schematic diagram of the process $e p \rightarrow e X p$. The kinematics of this reaction is described by the following variables:

- $Q^{2}=-q^{2}=-\left(k-k^{\prime}\right)^{2}$, the negative four-momentum squared of the virtual photon $\left(\gamma^{\star}\right)$, where $k\left(k^{\prime}\right)$ is the four-momentum of the incident (scattered) positron;

- $W^{2}=(q+P)^{2}$, the squared centre-of-mass energy of the photon-proton system, where $P$ is the four-momentum of the incident proton;

- $x=Q^{2} /(2 P \cdot q)$, the fraction of the proton momentum carried by the struck quark in the infinite-momentum frame (the Bjorken variable);

- $y=(q \cdot p) /(k \cdot p)$, the fraction of the positron energy transferred to the proton in the proton rest frame;

- $M_{X}^{2}=\left(q+P-P^{\prime}\right)^{2}$, the squared mass of the system $X$, where $P^{\prime}$ is the four-momentum of the scattered proton;

- $t=\left(P-P^{\prime}\right)^{2}$, the squared four-momentum transfer at the proton vertex;

- $\Phi$, the angle between the positron scattering plane and the proton scattering plane in the $\gamma^{\star} p$ centre-of-mass frame.

The variables $Q^{2}, W$ and $x$ are related by $x=Q^{2} /\left(Q^{2}+W^{2}-M_{p}^{2}\right)$, where $M_{p}$ is the proton mass.

The two dimensionless variables $x_{\mathbb{P}}$ and $\beta$ can be used instead of $M_{X}$ and $W$; they are given by

$$
\begin{gathered}
x_{\mathbb{P}}=\frac{\left(P-P^{\prime}\right) \cdot q}{P \cdot q}=\frac{Q^{2}+M_{X}^{2}-t}{Q^{2}+W^{2}-M_{p}^{2}}, \\
\beta=\frac{Q^{2}}{2\left(P-P^{\prime}\right) \cdot q}=\frac{Q^{2}}{Q^{2}+M_{X}^{2}-t} .
\end{gathered}
$$

They are related to $x$ by $x_{\mathbb{P}} \beta=x$. The variable $\beta$ is the Bjorken variable defined with respect to the four-momentum of the exchanged object. The variable $x_{\mathbb{P}}$ is often referred to as $\xi$ at hadron colliders.

The cross section for the reaction $e p \rightarrow e X p$ can be expressed in terms of the diffractive structure function $F_{2}^{D(4)}$ or of the reduced diffractive cross-section $\sigma_{r}^{D(4)}$, which are defined by the equation

$$
\begin{aligned}
\frac{d \sigma^{e p \rightarrow e X p}}{d \beta d Q^{2} d x_{\mathbb{P}} d t} & =\frac{4 \pi \alpha^{2}}{\beta Q^{4}}\left[1-y+\frac{y^{2}}{2\left(1+R^{D}\right)}\right] F_{2}^{D(4)}\left(\beta, Q^{2}, x_{\mathbb{P}}, t\right) \\
& =\frac{4 \pi \alpha^{2}}{\beta Q^{4}}\left[1-y+\frac{y^{2}}{2}\right] \sigma_{r}^{D(4)}\left(\beta, Q^{2}, x_{\mathbb{P}}, t\right) .
\end{aligned}
$$


The quantity $R^{D}=\sigma_{L}^{\gamma^{\star} p \rightarrow X p} / \sigma_{T}^{\gamma^{\star} p \rightarrow X p}$ is the ratio of the cross sections for longitudinally and transversely polarised virtual photons. The diiffractive longitudinal structure function, $F_{L}^{D}$, is related to $R^{D}$ via $F_{L}^{D}=F_{2}^{D} R^{D} /\left(1+R^{D}\right)$. The diffractive reduced cross section and the diffractive structure function coincide if $R^{D}=0$. Since $R^{D}$ has not been measured, the results are presented in terms of the diffractive reduced cross section.

The structure-function $F_{2}^{D(3)}\left(\beta, Q^{2}, x_{\mathbb{P}}\right)$ and the reduced cross-section $\sigma_{r}^{D(3)}\left(\beta, Q^{2}, x_{\mathbb{P}}\right)$ are obtained by integrating $F_{2}^{D(4)}$ and $\sigma_{r}^{D(4)}$ over $t$,

$$
\begin{aligned}
F_{2}^{D(3)}\left(\beta, Q^{2}, x_{\mathbb{P}}\right) & =\int F_{2}^{D(4)}\left(\beta, Q^{2}, x_{\mathbb{P}}, t\right) d t, \\
\sigma_{r}^{D(3)}\left(\beta, Q^{2}, x_{\mathbb{P}}\right) & =\int \sigma_{r}^{D(4)}\left(\beta, Q^{2}, x_{\mathbb{P}}, t\right) d t .
\end{aligned}
$$

The $\Phi$ dependence of the cross section is sensitive to the interference between the longitudinal and transverse amplitudes; the sensitivity to these interference terms disappears when $\Phi$ is integrated over. For unpolarised positrons and protons, the cross section can be decomposed as

$$
\frac{d \sigma^{e p \rightarrow e X p}}{d \Phi} \propto \sigma_{T}^{\gamma^{\star} p \rightarrow X p}+\epsilon \sigma_{L}^{\gamma^{\star} p \rightarrow X p}-2 \sqrt{\epsilon(1+\epsilon)} \sigma_{L T}^{\gamma^{\star} p \rightarrow X p} \cos \Phi-\epsilon \sigma_{T T}^{\gamma^{\star} p \rightarrow X p} \cos 2 \Phi,
$$

where $\sigma_{L T}^{\gamma^{\star} p \rightarrow X p}$ is due to the interference term between the amplitudes for longitudinal and transverse polarisations of the virtual photon and $\sigma_{T T}^{\gamma^{\star} p \rightarrow X p}$ is due to the interference term between the amplitudes for the two transverse polarisations. The parameter $\epsilon$ is defined as $\epsilon=2(1-y) /\left[1+(1-y)^{2}\right]$.

\section{Methods of selecting diffraction}

The kinematic properties of diffractive DIS, $e p \rightarrow e X p$, imply the following for the finalstate proton and the hadronic system $X$ :

- the proton suffers only a small perturbation and emerges from the interaction carrying a large fraction, $x_{L}$, of the incoming proton momentum. Diffractive events appear as a peak at $x_{L} \approx 1$, the diffractive peak, which at HERA extends down to $x_{L}$ of about 0.98 [14]. The absolute value of the four-momentum-transfer squared, $|t|$, is typically smaller than $1 \mathrm{GeV}^{2}$, with $\langle|t|\rangle \approx 0.15 \mathrm{GeV}^{2}[14]$;

- the difference in rapidity between the outgoing proton and the system $X$ is $\Delta \eta \approx$ $\ln \left(1 / x_{\mathbb{P}}\right)$ [15]. Since the cross section increases with decreasing $x_{\mathbb{P}}$, most of the events have small $x_{\mathbb{P}}$ and therefore a large separation in rapidity between the outgoing proton and any other hadronic activity in the event is expected; 
- conservation of momentum implies that the system $X$ must have a small mass $\left(M_{X}\right)$ with respect to the photon-proton centre-of-mass energy, since $1-x_{L} \gtrsim M_{X}^{2} / W^{2}$.

Conversely, in non-diffractive DIS, both the hadronic system associated with the struck quark, which is largely measured in the detector, and that of the proton remnant, which peaks in the forward direction, originate from the hadronisation of colour-connected states. In this case, the distribution of the final-state particles is governed by conventional fragmentation and particles are emitted roughly uniformly in rapidity along the $\gamma^{\star} p$ axis. Rapidity gaps are thus expected to be exponentially suppressed [16].

Therefore, to select diffractive events, either the final-state proton can be detected (LPS method) or the different characteristics of the system $X$ in diffractive and non-diffractive events (hadronic methods) can be exploited.

In the hadronic methods, events with high $M_{X}$ are not accessible since the non-diffractive background grows with $M_{X}$ and the rapidity gap becomes more and more forward (and eventually becomes confined to the beam-pipe). In addition, the measured cross section includes a contribution from events of the type $e p \rightarrow e X N$, in which the proton also dissociates into a low-mass state, $N$, separated from $X$ by a rapidity gap. Hadrons from the system $N$ can either escape undetected into the forward beam-pipe or fall into the detector acceptance. The mass of the system $N$ thus enters as an additional variable, and the observed particles must be assigned either to $N$ or to $X$. The contribution from proton-dissociative events needs to be estimated from a Monte Carlo (MC) simulation. While these limitations add to the systematic uncertainties, the statistical precision of the results is good due to the high acceptance of the central detector. Although the acceptance is not limited in $t$, no measurement of $t$ is possible because of the poor resolution of the transverse momentum of the system $X$.

High- $x_{L}$ samples selected by the LPS method have little or no background from protondissociative events or from non-diffractive DIS. They also allow a direct measurement of the variables $t, \Phi$ and $x_{\mathbb{P}}$, and give access to higher values of $M_{X}$. The statistical precision, however, is poorer than for the results obtained with the hadronic methods because of the small acceptance of the proton taggers - approximately $2 \%$ in the diffractive-peak region.

At HERA, several diffractive analyses based on the scattered proton measurement have been carried out [14,17-19]. Analyses with the hadronic methods have been performed with event selections based both on the presence of forward large rapidity gaps (LRG method) $[20,21]$ and on the shape of the mass distribution of the system $X$ ( $M_{X}$ method) $[2$, $18,22]$.

The results presented here are based both on the LPS method and on the LRG method; the same data have also been analysed with the $M_{X}$ method [2]. No attempt is made to isolate the Pomeron contribution, i.e. diffraction in the strict sense of the term, from 
contributions due to Reggeon and pion trajectories or non-diffractive DIS: only the contribution of proton-dissociative events is statistically subtracted.

\section{Reconstruction of the kinematic variables}

The identification of the scattered positron was based on a neural network [23] using information from the CAL. If the positron was at angles large enough to be inside the CTD acceptance, a CTD track was required. The variables $W$ and $Q^{2}$ were reconstructed using a combination of the electron method and the double angle method [24].

In the LPS analysis, the longitudinal $\left(p_{Z}\right)$ and transverse $\left(p_{X}, p_{Y}\right)$ momenta of the scattered proton were measured. The fractional energy of the outgoing proton, $x_{L}$, was defined as $x_{L}=p_{Z} / E_{p}$, where $E_{p}$ is the incoming proton energy. The variable $t$ is given by

$$
t=-\frac{p_{T}^{2}}{x_{L}}-\frac{\left(1-x_{L}\right)^{2}}{x_{L}} M_{p}^{2}
$$

where $p_{T}$ is the transverse momentum of the proton with respect to the incoming beam direction. The $t$ resolution was approximately $\sigma(t) / t=0.14 \mathrm{GeV} \sqrt{|t|}$, with $|t|$ in $\mathrm{GeV}^{2}$, and was dominated by the angular spread of the HERA proton beam. The proton and the positron momenta were used to determine $\Phi$, the azimuthal angle between the positron and proton scattering planes in the $\gamma^{\star} p$ frame. The resolution in $\Phi$ was approximately $0.2 \mathrm{rad}$.

The four-momentum of the system $X$ was determined from both calorimeter and tracking information. The energy deposits in the CAL and the track momenta measured in the CTD were combined into energy flow objects (EFOs) $[25,26]$ to obtain the best momentum resolution. The EFOs were corrected for energy losses due to the material of the detector. The mass $M_{X}$ was evaluated as

$$
M_{X, \mathrm{EFO}}^{2}=\left(\sum E_{i}\right)^{2}-\left(\sum p_{X, i}\right)^{2}-\left(\sum p_{Y, i}\right)^{2}-\left(\sum p_{Z, i}\right)^{2},
$$

where $\left(E_{i}, p_{X, i}, p_{Y, i}, p_{Z, i}\right)$ is the momentum four-vector of the $i^{\text {th }} \mathrm{EFO}$ and the sum runs over all EFOs not assigned to the scattered positron.

In the LPS analysis, the mass $M_{X}$ was also determined from the outgoing proton momentum as reconstructed in the LPS,

$$
M_{X, \mathrm{LPS}}^{2} \approx\left[1-x_{L}(1+x)\right] W^{2} .
$$


The best resolution on $M_{X}$ was obtained with $M_{X, \text { EFO }}$ when $M_{X}$ was small and with $M_{X, \mathrm{LPS}}$ when $M_{X}$ was large; $M_{X}$ was therefore reconstructed as

$$
M_{X}^{2}=w_{\mathrm{EFO}} M_{X, \mathrm{EFO}}^{2}+w_{\mathrm{LPS}} M_{X, \mathrm{LPS}}^{2},
$$

where the weights $w_{\mathrm{EFO}}$ and $w_{\mathrm{LPS}}$ are inversely proportional to the corresponding resolutions, and $w_{\mathrm{EFO}}+w_{\mathrm{LPS}}=1$. The resulting resolution was $\sigma\left(M_{X}\right) / M_{X}=0.35 / \sqrt{M_{X}}+0.08$, with $M_{X}$ in $\mathrm{GeV}$.

The variables $x_{\mathbb{P}}$ and $\beta$ were obtained from Eqs. (1) and (2), using the measured values of $Q^{2}, W, M_{X}$ and neglecting $t$, since $|t| \ll Q^{2}, M_{X}^{2}$.

The variable $y$ was reconstructed as $y_{\mathrm{JB}}=\sum \frac{\left(E_{i}-p_{Z, i}\right)}{2 E_{e}}$, where the sum runs over all EFOs not assigned to the scattered positron and $E_{e}$ is the energy of the incident positron ("Jacquet-Blondel method" [27]).

\section{Event selection}

The data used for the analysis were selected at the trigger level $[4,28]$ by requiring the presence of a scattered positron in the CAL. The trigger selection of the LRG data also required that the energy deposited in the FPC be smaller than $20 \mathrm{GeV}$. For the LPS data, a scattered proton was required in the LPS.

Offline, the following cuts were imposed:

- the energy of the scattered positron was required to be larger than $10 \mathrm{GeV}$. The position of the scattered positron was required to be within the fiducial region of the CAL. This was defined by a set of cuts [29] which removed regions where the inactive material was not adequately simulated or where the positron shower was not fully contained;

- the requirement $45<\left(E-P_{Z}\right)<65 \mathrm{GeV}$ was imposed. Here $E-P_{Z}=\sum\left(E_{i}-p_{Z, i}\right)$, with the summation running over all EFOs including the scattered positron. This cut reduced the size of the QED radiative corrections and the photoproduction background, where the scattered positron escaped undetected in the rear beam hole;

- the $Z$ coordinate of the interaction vertex, $Z_{\mathrm{vtx}}$, was required to be in the range $-50<Z_{\mathrm{vtx}}<50 \mathrm{~cm}$. Events without a measured vertex were assigned to the nominal interaction point;

- events with two electron candidates, of which at least one lacked an associated track, and which were back-to-back within $5^{\circ}$ in the azimuthal plane, were rejected. This cut removed the contribution of QED Compton scattering and deeply virtual Compton scattering. 
For the LRG sample, the presence of a rapidity gap of at least two units between the hadronic final-state $X$ and the outgoing proton was ensured by requiring that the energy deposited in the FPC, $E_{\mathrm{FPC}}$, be smaller than $1 \mathrm{GeV}$ and by demanding $\eta_{\max }^{\mathrm{CAL}}<3$. Here $\eta_{\max }^{\mathrm{CAL}}$ is the pseudorapidity of the most forward EFO with energy above $400 \mathrm{MeV}$ in the CAL. This combination of cuts suppressed background from non-diffractive and protondissociative processes.

The following requirements were used to select the scattered proton measured in the LPS:

- only events with $p_{X}<0$ were used as, for the present sample, the LPS acceptance for $p_{X}>0$ was low;

- the candidate proton was tracked along the beam line and was rejected if the distance of closest approach to the beam-pipe was less than $0.2 \mathrm{~cm}$. It was also rejected if the $X$ position of the track impact point at station S4 (upper part) was smaller than $-3.0 \mathrm{~cm}$. These cuts reduced the sensitivity of the acceptance to the uncertainty in the position of the beam-pipe apertures;

- beam-halo background was caused by scattered protons with energy close to that of the beam, originating from the interaction of a beam proton with the residual gas in the beam-pipe or with the collimators. A beam-halo proton may overlap with a standard non-diffractive DIS event. In this case, the proton measured in the LPS was uncorrelated with the activity in the central detector. This background was suppressed by the requirement that the sum of the energy and the longitudinal component of the total momentum measured in the CAL and the LPS be less than the kinematic limit of twice the incoming proton energy: $E+P_{Z} \simeq\left(E+P_{Z}\right)_{\mathrm{CAL}}+2 p_{Z}^{\mathrm{LPS}}<1860 \mathrm{GeV}$. This cut took into account the resolution of the measurement of $p_{Z}^{\text {LPS }}$. The residual beam-halo background and its subtraction are discussed in Section 8.2.

- the variable $t$ was required to be in the range $0.09<|t|<0.55 \mathrm{GeV}^{2}$. This cut eliminated regions where the LPS acceptance was small or rapidly changing.

The LRG analysis was further restricted to the regions $2<Q^{2}<305 \mathrm{GeV}^{2}, 40<W<$ $240 \mathrm{GeV}, 2<M_{X}<25 \mathrm{GeV}$ and $0.0002<x_{\mathbb{P}}<0.02$; the average $Q^{2}$ value is $13 \mathrm{GeV}^{2}$. For the LPS sample, the region selected was $2<Q^{2}<120 \mathrm{GeV}^{2}, 40<W<240 \mathrm{GeV}$, $2<M_{X}<40 \mathrm{GeV}$ and $0.0002<x_{\mathbb{I P}}<0.1$; the average $Q^{2}$ value for the LPS sample is $11 \mathrm{GeV}^{2}$. These selections yielded 708,851 events for the LRG analysis and 15,130 for the LPS analysis.

The LRG and LPS samples were collected simultaneously: $0.7 \%$ of the LRG events have a proton measured in the LPS and $35 \%$ of the LPS events are also contained in the LRG sample. 


\section{Monte Carlo simulation and acceptance corrections}

Monte Carlo simulations were used to correct the data for acceptance and detector effects. Diffractive events were simulated with the SATRAP generator [30], which is based on the saturation model of Golec-Biernat and Wüsthoff [31]. SATraP is embedded in the RAPGAP framework [32]. The version of SATRAP used here is identical to that used in a previous ZEUS publication [2], except for some reweighting to describe the measured distributions for the higher- $x_{\mathbb{P}}$ events, where Reggeon and pion exchanges become significant.

Diffractive events were also modelled with RAPGAP 2.08/06 [32], which is based on the model of Ingelman and Schlein [33] and assumes Regge factorisation: the structure function $F_{2}^{D(4)}$ is expressed as the sum of separately factorisable Pomeron and Reggeon contributions,

$$
F_{2}^{D(4)}\left(x_{\mathbb{P}}, t, \beta, Q^{2}\right)=f_{\mathbb{P}}\left(x_{\mathbb{P}}, t\right) F_{2}^{\mathbb{P}}\left(\beta, Q^{2}\right)+f_{\mathbb{R}}\left(x_{\mathbb{P}}, t\right) F_{2}^{\mathbb{R}}\left(\beta, Q^{2}\right) .
$$

The Pomeron and Reggeon fluxes, $f_{\mathbb{I P}, \mathbb{R}}\left(x_{\mathbb{P}}, t\right)$, were parameterised [1] as

$$
f_{\mathbb{P}, \mathbb{R}}\left(x_{\mathbb{P}}, t\right)=\frac{e^{b_{0}^{\mathbb{P}, \mathbb{R}} t}}{x_{\mathbb{P}}^{2 \alpha_{\mathbb{P}, \mathbb{R}}(t)-1}},
$$

with linear trajectories $\alpha_{\mathbb{P}, \mathbb{R}}(t)=\alpha_{\mathbb{P}, \mathbb{R}}(0)+\alpha_{\mathbb{P}, \mathbb{R}}^{\prime} t$, and with the values of the parameters taken from hadron-hadron data [34]. The Pomeron structure function $F_{2}^{I P}\left(\beta, Q^{2}\right)$ was taken from the $\mathrm{H} 1 \mathrm{dPDF}$ fit 2 [35]. The structure function $F_{2}^{\mathbb{R}}$ is unknown and was assumed to be that of the pion [36].

The process of QCD radiation was simulated differently in the two MC samples. In the SATRAP sample, a parton-shower model as implemented in MePs [37] was used. In the RAPGAP sample, higher-order QCD radiation was simulated with ARIADNE [38]. In both cases hadronisation was simulated with the Lund string model as implemented in JETSET $7.4[39,40]$.

Initial- and final-state QED radiation was simulated by using SATRAP or RAPGAP in conjunction with HeRACLES 4.6 [41]. The measurements were corrected for these effects and the cross sections are presented at the Born level.

The inclusive DIS events were simulated with DJANGOH 1.1 [42], using the CTEQ4D [43] parameterisation of the proton parton densities.

The Pythia 6.2 generator [44] was used to study the photoproduction background as well as the proton-dissociative contribution (see Section 8.1). Events in the proton-dissociative MC sample were reweighted such as to give a good description of all measured variables in the data. 
All generated events were passed through the standard ZEUS detector simulation, based on the GEANT 3.13 program [45], and through the trigger simulation package. The measurements were corrected for detector acceptance and resolution, and for radiative effects, with suitable combinations of the various MC models. A comparison of data and SATRAP for the LRG analysis is presented in Fig. 2 for the variables $\eta_{\max }^{\mathrm{CAL}}, M_{X}$, $Q^{2}, W, x_{\mathbb{P}}$ and $\beta$. The simulation is in satisfactory agreement with the data in the region of interest, indicated by vertical lines in the plots. A similar comparison for the LPS analysis is presented in Fig. 3 for the variables $x_{L},|t|, Q^{2}, W, M_{X}$, and $x_{\mathbb{P}}$. The simulation reproduces the data reasonably well. The diffractive peak is evident in Fig. 3a.

\subsection{Cross-section extraction}

The diffractive reduced cross section at a given point within a bin was obtained from the ratio of the background-subtracted number of events to the number of events in that bin predicted by SATRAP, multiplied by the Born-level reduced cross section used in SATRAP. Both the acceptance and the bin-centring corrections were thus taken from SATRAP.

For the LPS data, the cross section was directly measured only in a limited $t$ region and extrapolated to $0<|t|<1 \mathrm{GeV}^{2}$ assuming an exponential $t$-dependence, $d \sigma^{e p \rightarrow e X p} / d t \propto$ $\exp (-b|t|)$, with $b=7.0 \mathrm{GeV}^{-2}$. The effect of the extrapolation is to increase the cross section by a factor of about two; this factor is largely independent of kinematics. Data from elastic and proton-dissociative $p p$ and $\bar{p} p$ scattering indicate that the $t$ distribution is better described by the function $\exp \left(-b|t|+c t^{2}\right)$. For example, fits to the $\bar{p} p$ data at $\sqrt{s}=546 \mathrm{GeV}$ [46] yield $c=2.3 \pm 0.1 \mathrm{GeV}^{-4}$. In the extrapolation to the range $0<|t|<1 \mathrm{GeV}^{2}$, $c$ was nominally set to zero and changed up to $4 \mathrm{GeV}^{-4}$, yielding changes in the extrapolated cross section of up to $+9 \%$ (setting $c$ to $2 \mathrm{GeV}^{-4}$ changes the cross section by $+6 \%$ ). This effect was included in the normalisation uncertainty discussed in Section 9.

\section{Backgrounds}

\subsection{LRG analysis}

The main source of background in the LRG sample comes from events of the type $e p \rightarrow e X N$, in which the proton dissociates into a low-mass system, $N$. The protondissociative system can either escape entirely undetected in the forward beam-pipe or leak partially into the detector acceptance and therefore be measured by the FPC and 
the CAL. In the former case, the background events are included in the measured cross section, so that they bias the normalisation. As indicated by MC simulations, in the latter case most of the events are rejected by the FPC veto and by the $\eta_{\max }^{\mathrm{CAL}}$ cut.

The ratio of the LPS to the LRG results measures the fraction of proton-dissociative events in the LRG sample. The ratio is $0.76 \pm 0.01$ (stat. $)_{-0.02}^{+0.03}$ (syst. ${ }_{-0.05}^{+0.08}$ (norm.) and is independent of $Q^{2}, x_{\mathbb{P}}$ and $\beta$, as discussed in Section 10.4; the last uncertainty reflects the normalisation uncertainties, mostly due to the LPS data. The percentage of protondissociative events in the LRG sample is therefore $24 \pm 1$ (stat.) ${ }_{-3}^{+2}$ (syst.) ${ }_{-8}^{+5}$ (norm.)\%.

The contribution of proton-dissociative events to the measured cross sections was also estimated with PүтніA. A sample of proton-dissociative data was selected in two alternative ways:

- by requiring $\eta_{\max }^{\mathrm{CAL}}<2$ and $E_{\mathrm{FPC}}>1 \mathrm{GeV}$, and the remaining selection as described in Section 6 for the LRG events (this will be referred to as FPC PDISS sample);

- by adding to the selection described in Section 6 for the LRG events the requirement that a proton be measured in the LPS with $0.5<x_{L}<0.9$ (LPS PDISS sample).

The generated Pyтнia distributions for $M_{N}, M_{X}$ and $Q^{2}$ were reweighted to give the best description of these data samples, in particular the $E_{\mathrm{FPC}}$ distribution in the FPC PDISS sample and the $x_{L}$ distribution in the LPS PDISS sample. The median of the generated $M_{N}$ distribution in PythiA is $1.7 \mathrm{GeV}$. The median of the same distribution for the events which pass the LRG analysis cuts is $1.6 \mathrm{GeV}$. Figures $4 \mathrm{a}-\mathrm{b}$ show the comparison of PythiA with the proton-dissociative samples FPC and LPS PDISS as a function $E_{\mathrm{FPC}}$ and $x_{L}$, respectively. Also shown in Figs. 4c-e is the fraction of proton-dissociative events expected in the LRG sample as a function of $Q^{2}, \beta$ and $x_{\mathbb{P}}$. This fraction, obtained separately from the LPS and FPC PDISS samples, is constant at the level of $25 \%$ in both cases. The average of the FPC and LPS estimates provides a measurement of the proton-dissociative contribution to the LRG sample of $25 \pm 1$ (stat.) \pm 3 (syst.)\%, consistent with the ratio of the LPS to LRG results quoted above. The systematic uncertainty was estimated by varying the shape of the generated $M_{N}$ distribution, by changing the FPC cut as well as the $\eta_{\max }^{\mathrm{CAL}}$ cut and by taking into account the LPS normalisation uncertainty. The combination of the LPS and FPC PDISS samples covers nearly the whole $M_{N}$ spectrum, including the lowest $M_{N}$ values. This fact, along with the agreement with the LPS to LRG ratio, lends support to the present estimate of the proton dissociation background. A background contribution of $R_{\text {diss }}=25 \pm 1$ (stat.) \pm 3 (syst.)\% was therefore subtracted from the data ${ }^{2}$. Unless stated otherwise, all results are thus given for the reaction $e p \rightarrow e X p$, i.e. $M_{N}=M_{p}$.

\footnotetext{
${ }^{2}$ In terms of the ratio $R_{M_{X}}=1 /\left(1-R_{\text {diss }}\right)$ used elsewhere [14, 18], this background contribution corresponds to $R_{M_{X}}=1.33 \pm 0.02$ (stat.) \pm 0.05 (syst.).
} 
The Pythia generator was also used to evaluate the photoproduction background, which arises from low- $Q^{2}$ events in which the scattered positron escapes undetected in the rear direction and one of the final-state hadrons is misidentified as a positron. The largest contribution was found in the lowest $Q^{2}$ bin $\left(2<Q^{2}<3 \mathrm{GeV}^{2}\right)$, where it was about $1.2 \%$. This background was neglected.

The contribution of non-diffractive events, estimated with DJANGOH 1.1, was found to be roughly $10 \%$ in the highest $x_{\mathbb{P}}$ bin $\left(0.01<x_{\mathbb{P}}<0.02\right)$ and to decrease rapidly with decreasing $x_{\mathbb{P}}$. This background was not subtracted but bins in which the contribution was larger than $10 \%$ were rejected.

\subsection{LPS analysis}

The main background contribution in the LPS sample at high $x_{L}$ is given by proton beamhalo events. In such events, the proton detected in the LPS is not correlated with the measurements in the central detector. To estimate this background, the variable $E+P_{Z}$ (see Section 6) was used. For a signal event, this quantity should be equal to twice the initial proton energy, $1840 \mathrm{GeV}$, whereas for a beam-halo event it can exceed this value.

The $E+P_{Z}$ spectrum for the beam-halo events was constructed as a random combination of a generic DIS event (without the requirement of a track in the LPS) and a beam-halo track measured in the LPS, uncorrelated with the measurement in the main detector; here $P_{Z}$ includes the contribution of the energy deposition in the CAL and the proton momentum measured in the LPS. The resulting distribution, shown in Fig. 5 as the histogram, was normalised to the data for $E+P_{Z}>1925 \mathrm{GeV}$; this part of the distribution contains beam-halo events only. The background remaining after the cut at $E+P_{Z}<$ $1860 \mathrm{GeV}$ averages to $3.0 \pm 0.1$ (stat.)\%, and is a decreasing function of $x_{\mathbb{I P}}$. The results presented in this paper were corrected for this background.

The contribution from proton-dissociative events, $e p \rightarrow e X N$, studied with PyTHIA, was around $9 \%$ at $x_{\mathbb{I P}}=0.1$, decreasing rapidly with decreasing $x_{\mathbb{I P}}$. All results were corrected for this background. In the region $x_{\mathbb{P}}<0.02$, this background is negligible.

The photoproduction background was negligible.

\section{Systematic uncertainties}

The systematic uncertainties were estimated [14,29] by varying the cuts and by modifying the analysis procedure. The variations of the cuts were typically commensurate with the resolutions of the relevant variables. 
For each systematic check, the average effect on the cross section in the measured bins is indicated using the notation $\left(_{-b}^{+a}\right)$. Given a systematic check which produced an increase of the cross section in some bins and a decrease in some other bins, $a$ is the average increase and $b$ is the average decrease.

For both the LPS and LRG analyses, the following checks were performed:

- to evaluate the uncertainties due to the measurement of the scattered positron, the fiducial region for the impact position of the positron on the face of the CAL around the rear beam-pipe was enlarged by $1 \mathrm{~cm}\left({ }_{-0.2}^{+1.2}\right) \%$;

- the minimum energy of the positron was increased to $12 \mathrm{GeV}\left({ }_{-0.3}^{+0.2}\right) \%$;

- the minimum value of $E-P_{Z}$ was raised to $47 \mathrm{GeV}\left({ }_{-0.7}^{+1.0}\right) \%$;

- the cut on the $Z$ coordinate of the vertex was restricted to $-40<Z_{\mathrm{vtx}}<40 \mathrm{~cm}\left({ }_{-0.5}^{+0.5}\right) \%$;

- the effect of the uncertainty in the absolute calorimeter energy calibration was estimated by changing the energy scale by $\pm 2 \%$ in the data only, separately for the scattered positron $\left({ }_{-2.3}^{+2.5}\right) \%$ and the hadronic system $\left({ }_{-2.3}^{+2.3}\right) \%$;

- the $x_{\mathbb{P}}$ distribution in the MC was reweighted by a factor $\left(x_{\mathbb{P}} / 0.01\right)^{k}$, with $k$ varying between -0.03 and +0.03 ; the effect was $\left({ }_{-0.4}^{+0.4}\right) \%$ in the LPS analysis and $\left({ }_{-2.9}^{+3.0}\right) \%$ in the LRG analysis, the difference being mainly due to the correlation between the $x_{\mathbb{P}}$ and $\eta_{\max }$ variables.

For the LRG analysis, the following specific checks were also performed:

- the FPC energy cut was lowered to $0.7 \mathrm{GeV}\left({ }_{-0.4}^{+0.5}\right) \%$;

- the energy threshold on the most forward EFO used to reconstruct $\eta_{\max }^{\mathrm{CAL}}$ was lowered to $300 \mathrm{MeV}\left({ }_{-0.3}^{+0.4}\right) \%$ and increased to $500 \mathrm{MeV}\left({ }_{-0.4}^{+0.2}\right) \%$.

For the LPS analysis, the following specific checks were also performed:

- the cut on the minimum distance of approach to the beam-pipe was increased by $0.03 \mathrm{~cm}\left({ }_{-0.8}^{+0.4}\right) \%$;

- the $t$ range was restricted to $0.1<|t|<0.5 \mathrm{GeV}^{2}\left({ }_{-5.1}^{+4.1}\right) \%$;

- the proton-dissociative background was varied by $\pm 30 \%\left({ }_{-0.9}^{+0.9}\right) \%$;

- the value of the $t$-slope in the MC was changed by $\pm 1 \mathrm{GeV}^{-2}\left({ }_{-2.9}^{+4.0}\right) \%$;

- the $\Phi$ distribution in the MC was reweighted by a factor $(1+k \cos \Phi)$, with $k$ varying between -0.15 and $+0.15\left({ }_{-0.9}^{+1.0}\right) \%$;

- the intrinsic transverse-momentum spread of the proton beam at the interaction point was increased by $5 \mathrm{MeV}$ in the horizontal plane and $10 \mathrm{MeV}$ in the vertical plane $\left(\begin{array}{l}+1.6 \\ -1.9\end{array}\right) \%$. 
The total systematic uncertainty for each bin was taken as the quadratic sum of the individual contributions. The effect of using the generator RAPGAP for the acceptance corrections instead of SATRAP was estimated $\left({ }_{-8.6}^{+9.3}\right) \%$ but not included in the error bars as RAPGAP was found to provide a poor description of the data distributions.

For the LPS data, there is also an overall uncertainty of $\pm 7 \%$ which originates mostly from the uncertainty of the simulation of the proton-beam optics - largely independent of the kinematic variables, and therefore taken as a normalisation uncertainty. It also includes the uncertainty on the integrated luminosity $( \pm 2.25 \%)$.

In the LPS results integrated over $t\left(\sigma_{r}^{D(3)}\right.$ and $\left.d \sigma / d \Phi\right)$, an additional $+9 \%$ uncertainty is present, due to the extrapolation from the measured to the full $t$ range (see Section 7.1). The overall LPS normalisation uncertainty then becomes ${ }_{-7}^{+11} \%$.

For the LRG data, the uncertainty on the integrated luminosity $( \pm 2.25 \%)$ and that on the proton dissociation background $( \pm 4 \%)$ give an overall normalisation uncertainty of $\pm 5 \%$.

\section{Results}

The results in this section are presented as follows. The cross-section $d \sigma^{e p \rightarrow e X p} / d t$ in the region $0.09<|t|<0.55 \mathrm{GeV}^{2}$ is discussed first. The data are then integrated over $t$ and extrapolated to the range $0<|t|<1 \mathrm{GeV}^{2}$, as discussed in Section 7.1. The resulting cross sections are presented as a function of $\Phi$ in Section 10.2, where the sensitivity of the present data to the helicity structure of the reaction $e p \rightarrow e X p$ is discussed. The LPS data were used for both the $t$ and the $\Phi$ cross sections. In Sections 10.3 and 10.4, the data are presented in terms of the diffractive reduced cross sections, $\sigma_{r}^{D(4)}$ and $\sigma_{r}^{D(3)}$. The former was measured, for the first time, in two bins of $t$, and was obtained from the LPS data. The latter was obtained both from the LPS data, after integration over $t$, and from the LRG data. In Section 10.5, the $x_{\mathbb{I P}}$ dependence of $\sigma_{r}^{D(4)}$ and $\sigma_{r}^{D(3)}$ is used to extract the intercept of the Pomeron trajectory, $\alpha_{\mathbb{P}}(0)$, the quantity that, in Regge phenomenology, determines the energy dependence of the total hadron-hadron cross section [1].

The results for the LPS sample extend up to $x_{\mathbb{P}}=0.1$. In this paper, the LPS data in the diffractive-peak region are often compared with those at high $x_{\mathbb{P}}$. For this purpose, the value $x_{\mathbb{P}}=0.01$ was chosen as the transition between the high- and low- $x_{\mathbb{P}}$ bins, such that the low- $x_{\mathbb{I P}}$ bins are dominated by diffractive-peak events, while at higher $x_{\mathbb{P}}$ Reggeon and pion exchanges are important [3]. This choice is somewhat restrictive, since the diffractive peak extends well below $x_{L}=0.99$, see Fig. 3a. In the region $x_{\mathbb{P}}<0.01$, the contribution from non-Pomeron exchanges is less than $10 \%$. The average value of $x_{\mathbb{P}}$ is 0.003 for $x_{\mathbb{P}}<0.01$ and 0.043 for $0.01<x_{\mathbb{P}}<0.1$. 


\section{$10.1 \quad t$ dependence}

The differential cross-section $d \sigma^{e p \rightarrow e X p} / d t$, obtained from the LPS data in the kinematic range $2<Q^{2}<120 \mathrm{GeV}^{2}, 2<M_{X}<40 \mathrm{GeV}, 40<W<240 \mathrm{GeV}$ and $0.09<|t|<$ $0.55 \mathrm{GeV}^{2}$, both for $0.0002<x_{\mathbb{P}}<0.01$ (diffractive-peak region) and $0.01<x_{\mathbb{P}}<0.1$, is presented in Fig. 6 and Table 1.

The data were fitted with the single-exponential function $d \sigma^{e p \rightarrow e X p} / d t \propto e^{-b|t|}$. The value of the slope parameter, $b$, obtained from the fit in the region $0.0002<x_{\mathbb{P}}<0.01$ is $b=$ $7.0 \pm 0.3 \mathrm{GeV}^{-2}$, with $\chi^{2} / \mathrm{ndf}=1.8(\mathrm{ndf}=2)$ when statistical and systematic uncertainties summed in quadrature are used in the fit. This result agrees with the previous ZEUS result [14]. In the high- $x_{\mathbb{P}}$ region, $0.01<x_{\mathbb{P}}<0.1$, the fit gives $b=6.9 \pm 0.3 \mathrm{GeV}^{-2}$ with $\chi^{2} / \mathrm{ndf}=1.1$, again when the quadratic sum of statistical and systematic uncertainties is used.

The values of the $t$-slope in different bins of $Q^{2}, M_{X}$ and $x_{I P}$ are shown in Fig. 7 and given in Table 2. The diffractive-peak as well as the high- $x_{\mathbb{P}}$ region are shown. The $t$-slope does not depend on $Q^{2}, M_{X}$ and $x_{\mathbb{P}}$ in the measured regions. The lack of $Q^{2}$ dependence in a wide range of $Q^{2}$ as well as a value of $b$ much larger than that measured in hard diffraction (as discussed in a recent ZEUS publication [47]) suggests that inclusive diffractive dissociation in DIS is a soft process.

\section{$10.2 \Phi$ dependence}

The azimuthal angle, $\Phi$, between the positron and proton scattering planes is sensitive to the helicity structure of the reaction $e p \rightarrow e X p$, as shown explicitly in Eq. (4). To reduce the $\Phi$ dependence of the acceptance, an additional radial cut of $18 \mathrm{~cm}$ was imposed on the impact point of the scattered positron at the RCAL surface, along with the restriction $Q^{2}>4 \mathrm{GeV}^{2}$. These cuts were only applied for the $\Phi$ analysis. The LPS data were used. The $\Phi$ distribution for the two ranges $0.0002<x_{\mathbb{P}}<0.01$ and $0.01<x_{\mathbb{P}}<0.1$ is presented in Figs. 8a-b and Table 3.

The distributions were fitted to the form

$$
\frac{d \sigma^{e p \rightarrow e X p}}{d \Phi} \propto 1+A_{L T} \cos \Phi+A_{T T} \cos 2 \Phi,
$$

where $A_{L T}$ and $A_{T T}$ are proportional to $\sigma_{L T}^{\gamma^{\star} p \rightarrow X p}$ and $\sigma_{T T}^{\gamma^{\star} p \rightarrow X p}$, respectively. The values of the azimuthal asymmetries are

$$
\begin{aligned}
& \left.A_{L T}=-0.036 \pm 0.036(\text { stat. })_{-0.014}^{+0.016} \text { (syst. }\right) \\
& \left.A_{T T}=-0.030 \pm 0.037 \text { (stat. }\right)_{-0.006}^{+0.022} \text { (syst.) }
\end{aligned}
$$


and

$$
\begin{aligned}
& \left.A_{L T}=+0.051 \pm 0.024 \text { (stat. }\right)_{-0.011}^{+0.012} \text { (syst.) }, \\
& \left.A_{T T}=-0.010 \pm 0.024 \text { (stat. }\right)_{-0.009}^{+0.010} \text { (syst.) }
\end{aligned}
$$

for the ranges $0.0002<x_{\mathbb{P}}<0.01$ and $0.01<x_{\mathbb{P}}<0.1$, respectively.

The interference terms between the longitudinal and transverse amplitudes and between the two transverse amplitudes are thus small in the measured kinematic range, both in the diffractive-peak region and at higher- $x_{\mathbb{P}}$ values, suggesting that the helicity structure of the reaction $e p \rightarrow e X p$ is similar for both Pomeron and sub-leading Regge trajectories.

Figure 9 presents $A_{L T}$ and $A_{T T}$ as a function of $x_{\mathbb{P}}$, and, for $x_{\mathbb{P}}<0.01$, as a function of $\beta, t$ and $Q^{2}$. The asymmetries, also given in Tables 4 and 5 , are consistent with zero.

The measured values of $A_{L T}$ can be compared with the results obtained in the exclusive electroproduction of $\rho^{0}$ mesons, $e p \rightarrow e \rho^{0} p$, in which the hadronic final state, $X$, consists of a $\rho^{0}$ meson only. In this case, $A_{L T}=-\sqrt{2 \epsilon(1+\epsilon)} \cdot\left(r_{00}^{5}+2 r_{11}^{5}\right)=$ $-0.256 \pm 0.030$ (stat.) ${ }_{-0.022}^{+0.032}$ (syst.), where $r_{00}^{5}$ and $r_{11}^{5}$ are two of the $\rho^{0}$ spin-density matrix elements [47]. The present data therefore show that the asymmetry is smaller for inclusive scattering than for exclusive $\rho^{0}$ electroproduction.

There are numerous pQCD-based predictions for the behaviour of $A_{L T}$ [48-51] in the diffractive peak region, mostly for $\beta \gtrsim 0.9$, where the asymmetry is expected to be largest, reflecting the dominance of $\sigma_{L}^{\gamma^{\star} p \rightarrow X p}$ at large $\beta$ values. In all calculations, backto-back configurations, i.e. $A_{L T}<0$, are favoured. There is no indication of such a behaviour in the present data; the statistics at high $\beta$ is however limited. The asymmetry is expected to be close to zero at low $\beta$, in agreement with the data.

\subsection{The reduced cross-section $\sigma_{r}^{D(4)}$}

The LPS data are presented in Fig. 10 in terms of the reduced cross-section $\sigma_{r}^{D(4)}$ in two $t$ bins, $0.09<|t|<0.19 \mathrm{GeV}^{2}$ and $0.19<|t|<0.55 \mathrm{GeV}^{2}$, with $\langle|t|\rangle=0.13 \mathrm{GeV}^{2}$ and $\langle|t|\rangle=0.3 \mathrm{GeV}^{2}$, respectively. The figure shows $x_{\mathbb{P}} \sigma_{r}^{D(4)}$, also given in Tables 6 and 7 , as a function of $x_{\mathbb{I P}}$ for different values of $\beta, Q^{2}$ and $|t|$.

At low $x_{\mathbb{P}}$ and high $\beta, x_{\mathbb{P}} \sigma_{r}^{D(4)}$ decreases with increasing $x_{\mathbb{P}}$. At medium $x_{\mathbb{P}}$ and $\beta$, the dependence of $x_{\mathbb{P}} \sigma_{r}^{D(4)}$ on $x_{\mathbb{P}}$ is weak, whereas at high $x_{\mathbb{P}}$ and low $\beta, x_{\mathbb{P}} \sigma_{r}^{D(4)}$ increases with increasing $x_{\mathbb{P}}$. The behaviour observed at high $x_{\mathbb{P}}$ and low $\beta$ can be ascribed to Reggeon and pion exchange. The Regge fit described in Section 10.5 indicates that the shape of the $x_{\mathbb{P}}$ dependence is the same in the two $t$ bins. 


\subsection{The reduced cross-section $\sigma_{r}^{D(3)}$}

The reduced cross section, $x_{\mathbb{P}} \sigma_{r}^{D(3)}$, obtained with the LPS method, is shown in Fig. 11 and given in Table 8 as a function of $x_{\mathbb{P}}$ for different values of $\beta$ and $Q^{2}$. The same features already discussed for $x_{\mathbb{P}} \sigma_{r}^{D(4)}$ are seen here. The LPS data are also shown in Fig. 12 compared with the H1 data from the H1 forward proton spectrometer (FPS) [19]. For this plot, the analysis was redone using the same $Q^{2}$ and $\beta$ bins as H1, thus avoiding extrapolation uncertainties. The agreement is satisfactory.

The LRG data, corrected to $M_{N}=M_{p}$ as discussed in Sect. 8.1, are presented in Figs. 13 and 14 in terms of the reduced cross section, $\sigma_{r}^{D(3)}$. The figures show $x_{\mathbb{P}} \sigma_{r}{ }^{D(3)}$, also given in Table 9, as a function of $x_{\mathbb{P}}$ for different values of $\beta$ and $Q^{2}$. The behaviour of $x_{\mathbb{P}} \sigma_{r}^{D(3)}$ is similar to that observed above for $x_{\mathbb{P}} \sigma_{r}^{D(4)}$, with an increase with decreasing $x_{\mathbb{P}}$ at low $x_{\mathbb{P}}$ and high $\beta$.

Figure 15 shows the ratio of the $\sigma_{r}^{D(3)}$ values obtained with the LPS method to those obtained with the LRG method, before the subtraction of the proton-dissociative contribution. The ratio is independent of $x_{\mathbb{P}}, Q^{2}$ and $\beta$ and averages $0.76 \pm 0.01$ (stat.) ${ }_{-0.02}^{+0.03}$ (syst.). The $x_{\mathbb{P}}, Q^{2}$ and $\beta$ dependences of $\sigma_{r}^{D(3)}$ measured with the LPS method and the LRG method are consistent in the region of overlap. The normalisation difference is ascribed to the proton-dissociative contribution in the LRG sample, as discussed in Section 8.1.

The LRG data, corrected to $M_{N}<1.6 \mathrm{GeV}$ as described below, are shown as a function of $Q^{2}$ in different $\beta$ bins for $x_{\mathbb{P}}=0.0003, x_{\mathbb{P}}=0.001, x_{\mathbb{P}}=0.003$ and $x_{\mathbb{P}}=0.01$ in Figs. 16 and 17. The values of $x_{\mathbb{P}} \sigma_{r}^{D(3)}$ exhibit a logarithmic rise with $Q^{2}$ for all $\beta$ values except in the lowest $x_{\mathbb{P}}$ bin $\left(x_{\mathbb{P}}=0.0003\right)$ and in the highest $\beta$ bin $(\beta=0.8)$. The rise observed even at high $\beta$ suggests that the diffractive PDFs of the proton are gluon-dominated.

In Figs. 16 and 17 the LRG results are also compared with those of the H1 Collaboration [20], similarly obtained with the LRG method. The ZEUS results are measured in the H1 $\beta$ and $x_{\mathbb{P}}$ bins; they are corrected to $M_{N}<1.6 \mathrm{GeV}$, as are the H1 data. The correction to $M_{N}<1.6 \mathrm{GeV}$ for the present data, before the subtraction of the protondissociation background, was estimated with PүтніA to be $0.91 \pm 0.07$, independent of $\beta, Q^{2}$ and $x_{\mathbb{P}}$. Therefore, the ZEUS results in Figs. 16 and 17 were scaled down by 0.91. With some exceptions, the shape agreement is reasonable. The ZEUS data are higher than the $\mathrm{H} 1$ data by $13 \%$ on average, as estimated with a global fit to data for $Q^{2}>6 \mathrm{GeV}^{2}$. This normalisation discrepancy is consistent with the $8 \%$ uncertainty on the proton-dissociation correction of $0.91 \pm 0.07$ combined with the $7 \%$ relative normalisation uncertainty between the two data sets ( $\pm 7 \%$ for $\mathrm{H} 1$ and $\pm 2.25 \%$ for ZEUS).

Figures 16 and 17 are combined in Fig. 18 where the H1 and ZEUS reduced cross sections, the latter scaled down by the factor $1-0.13=0.87$ just described, are shown as a function 
of $Q^{2}$ in different $\beta$ and $x_{\mathbb{P}}$ bins. The result of the NLO QCD fit "H1 2006 fit B" [20] is also shown. At fixed $\beta$, the $Q^{2}$ dependence of the two data sets, taken together, is different for different $x_{\mathbb{P}}$ values. Therefore, the data cannot be described by a single factorisable Regge contribution.

Figures 19 and 20 compare the LRG results, corrected to $M_{N}=M_{p}$, to those obtained with the $M_{X}$ method, referred to as FPC I [22] and FPC II [2]. The LRG and FPC II data were collected simultaneously; the two samples overlap by about $75 \%$. The LRG results were recalculated in the bins used for the $M_{X}$-method results. The latter are for $M_{N}<2.3 \mathrm{GeV}$, but have been normalised here to the LRG results. The scaling factor applied to the $M_{X}$ results was $0.83 \pm 0.04$, estimated with a global fit to the present data and the $M_{X}$ data; this factor quantifies the amount of residual proton-dissociative background in the $M_{X}$ method. The overall agreement between the two measurements is reasonable. The different $x_{\mathbb{P}}$ dependence, more evident at low $Q^{2}$, may be ascribed to the fact that in the $M_{X}$ results the contribution of the Reggeon and pion trajectories is suppressed. In the low- $Q^{2}$ region, the $Q^{2}$ behaviour is somewhat different in the two data sets, with the $M_{X}$-method results decreasing faster with $Q^{2}$ than the LRG results.

\subsection{Extraction of the Pomeron trajectory}

In the framework of Regge phenomenology, the $x_{\mathbb{P}}$ dependence of $F_{2}^{D(4)}$ and $F_{2}^{D(3)}$ is related to the intercept of the Pomeron trajectory, the parameter that drives the energy dependence of the total hadron-hadron cross section at high energies [1]. The Pomeron intercept in soft hadronic interactions is $1.096_{-0.009}^{+0.012}$ [52]. However, the same parameter is significantly larger in the diffractive production of heavy vector mesons, notably in $J / \psi$ photoproduction (see e.g. [21,53]), reflecting the rapid rise of the cross section with $W$. This is a consequence of the increase of the parton densities in the proton at low $x$, which drives the rise of the cross section with decreasing $x$, and hence with decreasing $x_{\mathbb{P}}$ (since $x_{\mathbb{P}} \propto 1 / W^{2} \propto x$ ). The slope of the Pomeron trajectory, $\alpha_{\mathbb{P}}^{\prime}$, is smaller in the diffractive production of vector mesons [53] than in soft hadron-hadron collisions, where $\alpha_{\mathbb{P}}^{\prime}=0.25 \mathrm{GeV}^{-2}[15]$. It is therefore interesting to determine if such deviations from the behaviour of the hadron-hadron data are also apparent in the inclusive diffractive dissociation of virtual photons.

Following the Regge factorisation assumption (see Eq. (7)), the data of Fig. 10 were fitted to the form

$$
F_{2}^{D(4)}=f_{\mathbb{P}}\left(x_{\mathbb{P}}, t\right) \cdot F_{2}^{\mathbb{P}}\left(\beta, Q^{2}\right)+n_{\mathbb{R}} \cdot f_{\mathbb{R}}\left(x_{\mathbb{P}}, t\right) \cdot F_{2}^{\mathbb{R}}\left(\beta, Q^{2}\right),
$$

where $n_{\mathbb{R}}$ is a normalisation term. It was assumed that $F_{2}^{D(4)}=\sigma_{r}^{D(4)}$ and the fit was limited to $y<0.5$ to reduce the influence of $F_{L}^{D}$. The Pomeron and the Reggeon fluxes 
were parameterised as [1]

$$
f_{\mathbb{P}}\left(x_{\mathbb{P}}, t\right)=\frac{e^{B_{\mathbb{P}} t}}{x_{\mathbb{P}}^{2 \alpha_{\mathbb{P}}(t)-1}} \quad \text { and } \quad f_{\mathbb{R}}\left(x_{\mathbb{P}}, t\right)=\frac{e^{B_{\mathbb{R}} t}}{x_{\mathbb{P}}^{2 \alpha_{\mathbb{R}}(t)-1}}
$$

and the Pomeron and Reggeon trajectories were both assumed to be linear. The fitted parameters were the Pomeron trajectory, $\alpha_{\mathbb{P}}(0)$ and $\alpha_{\mathbb{P}}^{\prime}$, the intercept of the Reggeon trajectory, $\alpha_{\mathbb{R}}(0)$, the slope, $B_{\mathbb{P}}$, and the Reggeon normalisation term, $n_{\mathbb{R}}$. The Reggeon structure function, $F_{2}^{\mathbb{R}}\left(\beta, Q^{2}\right)$, was taken to be equal to the pion structure function as parameterised by GRV [54-56]. The slope $B_{\mathbb{R}}$ was fixed to $2.0 \mathrm{GeV}^{-2}$, taken from hadronhadron data, and the slope of the Reggeon trajectory, $\alpha_{\mathbb{R}}^{\prime}$, was fixed to $0.9 \mathrm{GeV}^{-2}$. The lines in Fig. 10 show the result of the fit. The results for the fit parameters are given in Table 10.

The model uncertainty reflects the effect of $R^{D}$, which was varied between 0 and 1 , and that of the parameterisation of the pion structure function, which was changed from that of GRV to that of Owens [36]. The quality of the fit is good. The Pomeron intercept is consistent with that of the soft Pomeron. The result for $\alpha_{\mathbb{P}}^{\prime}$ is significantly lower than $\alpha_{\mathbb{P}}^{\prime}=0.25 \mathrm{GeV}^{-2}$; it agrees with the result recently found by the H1 Collaboration [20] as well as with the values found in the diffractive production of vector mesons [53]. The Reggeon intercept is higher than the expectation of 0.5475 based on the Donnachie and Landshoff fits to the $p p, \bar{p} p, K p, \pi p$ and $\gamma p$ total cross section data [34]. Allowing for maximal interference between the Pomeron and Reggeon amplitudes also gives a good fit.

A similar fit was performed to the $\sigma_{r}^{D(3)}$ LRG points. The result of the fit is shown in Figs. 13 and 14 and the parameters, both those kept fixed and those obtained from the fit, are summarised in Table 11. The first uncertainty is that from the fit, in which the quadratic sum of statistical and systematic uncertainties was used. The model uncertainty reflects the variation of $\alpha_{\mathbb{P}}^{\prime}$ between 0 and $0.1 \mathrm{GeV}^{-2}$ and that of $\alpha_{\mathbb{R}}(0)$ between 0.55 and 0.75; in addition, as for the fit to the LPS data, $R^{D}$ was varied between 0 and 1 , and the pion structure function parameterisation was changed from that of GRV to that of Owens [36]. Here again, the fit was limited to $y<0.5$. The quality of the fit is very good.

Figure 21 shows $\alpha_{\mathbb{P}}(0)$ as a function of $Q^{2}$; it was obtained with a fit to the LRG data in bins of $Q^{2}$, similar to that described earlier for the full $Q^{2}$ range. The Reggeon normalisation term, $n_{\mathbb{R}}$, was fixed to the value $n_{\mathbb{R}}=2.6 \pm 0.3$, extracted from a combined Regge fit to the LPS and LRG results in the full $Q^{2}$ range. The LPS result and those obtained with the $M_{X}$ method, FPC I [22] and FPC II [2], are also shown. In the region explored, the present data do not exhibit a significant dependence on $Q^{2}$. The agreement with the $M_{X}$-method results is fair. 


\section{Summary}

Measurements have been presented of the reaction $e p \rightarrow e X p$ obtained by requiring a large rapidity gap in the forward direction (LRG sample) or the detection of a proton in the leading proton spectrometer (LPS sample). The kinematic region is $2<Q^{2}<305 \mathrm{GeV}^{2}$ (LRG) or $2<Q^{2}<120 \mathrm{GeV}^{2}$ (LPS), $40<W<240 \mathrm{GeV}, 2<M_{X}<25 \mathrm{GeV}$ (LRG) or $2<M_{X}<40 \mathrm{GeV}$ (LPS), $0.0002<x_{\mathbb{P}}<0.02(\mathrm{LRG})$ or $0.0002<x_{\mathbb{P}}<0.1$ (LPS) and $0.09<|t|<0.55 \mathrm{GeV}^{2}$ (LPS).

The LPS data are presented in terms of the $t$ and $\Phi$ dependences of the cross section, as well as of the $x_{\mathbb{P}}, Q^{2}, \beta$ and $t$ dependences of the reduced diffractive cross section, $\sigma_{r}^{D(4)}$. The $t$ dependence of the cross section is approximately exponential, with a $t$-slope $b=7.0 \pm 0.4 \mathrm{GeV}^{-2}$. The slope is independent of $Q^{2}, M_{X}$ and $x_{\mathbb{P}}$. The lack of $Q^{2}$ dependence and the value of $b$ much larger than that measured in hard diffraction suggest that this is a soft process. There is no significant $\Phi$ dependence of the cross section. The cross-section $\sigma_{r}^{D(4)}$ was measured for the first time in two $t$ bins and was found to have the same $x_{\mathbb{P}}$ dependence in the two bins.

The reduced cross-section $\sigma_{r}^{D(3)}$ was measured using both the LRG and LPS data. Consistent results were found for the shape. The normalisation difference of about $25 \%$ is ascribed to the proton-dissociative contribution in the LRG data. Within the normalisation uncertainties the results agree reasonably well with the H1 measurements [20]. The comparison with the ZEUS $M_{X}$-method results $[2,22]$ indicates that the latter have a residual proton-dissociative contribution of $17 \%$; the shape agreement is good, especially at low $x_{\mathbb{P}}$. A Regge fit to $\sigma_{r}^{D(3)}$ supports the $Q^{2}$ independence of $\alpha_{\mathbb{P}}(0)$.

\section{Acknowledgements}

We thank the DESY Directorate for their support and encouragement. We are grateful for the support of the DESY computing and network services. We are specially grateful to the HERA machine group: collaboration with them was crucial to the successful installation and operation of the leading proton spectrometer. The design, construction and installation of the ZEUS detector have been made possible by the ingenuity and effort of many people who are not listed as authors. It is a pleasure to thank A.D. Martin, M.G. Ryskin and G. Watt for many useful discussions. 


\section{References}

[1] P.D.B. Collins, An Introduction to Regge Theory and High Energy Physics, Cambridge University Press, Cambridge (1977);

S. Donnachie et al., Camb. Monogr. Part. Phys. Nucl. Phys. Cosmol. 19 (2002) 1.

[2] ZEUS Coll., S. Chekanov et al., DESY Report DESY-08-011, arXiv:0802.3017v2 [hep-ex], accepted by Nucl. Phys. B.

[3] K. Golec-Biernat, J. Kwiecinski and A. Szczurek, Phys. Rev. D 56, 3955 (1997).

[4] ZEUS Coll., U. Holm (ed.), The ZEUS Detector, Status Report (unpublished), DESY (1993), available on http://www-zeus.desy.de/bluebook/bluebook.html.

[5] ZEUS Coll., M. Derrick et al., Phys. Lett. B 293, 465 (1992).

[6] N. Harnew et al., Nucl. Inst. Meth. A 279, 290 (1989);

B. Foster et al., Nucl. Phys. Proc. Suppl. B 32, 181 (1993);

B. Foster et al., Nucl. Inst. Meth. A 338, 254 (1994).

[7] M. Derrick et al., Nucl. Inst. Meth. A 309, 77 (1991);

A. Andresen et al., Nucl. Inst. Meth. A 309, 101 (1991);

A. Caldwell et al., Nucl. Inst. Meth. A 321, 356 (1992);

A. Bernstein et al., Nucl. Inst. Meth. A 336, 23 (1993).

[8] A. Bamberger et al., Nucl. Inst. Meth. A 401, 63 (1997).

[9] ZEUS Coll., S. Chekanov et al., Eur. Phys. J. C 21, 443 (2001).

[10] A. Dwurazny et al., Nucl. Inst. Meth. A 277, 176 (1989).

[11] ZEUS Coll., A. Bamberger et al., Nucl. Inst. Meth. A 450, 235 (2000).

[12] ZEUS Coll., M. Derrick et al., Z. Phys. C 73, 253 (1997).

[13] J. Andruszków et al., Technical Report DESY-92-066, DESY, 1992;

ZEUS Coll., M. Derrick et al., Z. Phys. C 63, 391 (1994);

J. Andruszków et al., Acta Phys. Pol. B 32, 2025 (2001).

[14] ZEUS Coll., S. Chekanov et al., Eur. Phys. J. C 38, 43 (2004).

[15] V. Barone and E. Predazzi, High-Energy Particle Diffraction, Springer Verlag, Heidelberg (2002).

[16] HRS Coll., M. Derrick et al., Z. Phys. C 35, 323 (1987).

[17] ZEUS Coll., J. Breitweg et al., Eur. Phys. J. C 1, 81 (1997).

[18] ZEUS Coll., S. Chekanov et al., Eur. Phys. J. C 25, 169 (2002).

[19] H1 Coll., A. Aktas et al., Eur. Phys. J. C48, 749 (2006). 
[20] H1 Coll., A. Aktas et al., Eur. Phys. J. C 48, 715 (2006).

[21] H. Abramowicz, Int. J. Mod. Phys. A 15 S1, 495 (2000).

[22] ZEUS Coll., S. Chekanov et al., Nucl. Phys. B 713, 3 (2005).

[23] H. Abramowicz, A. Caldwell and R. Sinkus, Nucl. Inst. Meth. A 365, 508 (1995).

[24] S. Bentvelsen, J. Engelen and P. Kooijman, Proc. Workshop on Physics at HERA, W. Buchmüller and G. Ingelman (eds.), Vol. 1, p. 23. DESY, Hamburg, Germany (1992).

[25] ZEUS Coll., J. Breitweg et al., Eur. Phys. J. C 6, 43 (1999).

[26] G. Briskin, Ph.D. Thesis, Tel Aviv University, DESY-THESIS-1998-036 (1988).

[27] F. Jacquet and A. Blondel, Proc. Study of an ep Facility for Europe, U. Amaldi (ed.), p. 391. DESY, Hamburg, Germany (1979).

[28] W.H. Smith et al., Nucl. Instr. Meth. A 355, 278 (1995);

W.H. Smith, K. Tokushuku, and L.W. Wiggers, Proc. of the 10th International Conference on Computing in High Energy Physics 1992 (CHEP 92), C. Verkerk and W. Wojcik (eds.). CERN, Geneva, Switzerland (1992).

[29] J. Lukasik, Ph.D. Thesis, Cracow University, DESY-THESIS-2007-038 (2007).

[30] H. Kowalski, Proc. of the Ringberg Workshop: New Trends in HERA Physics 1999, G. Grindhammer, B.A. Kniehl and G. Kramer (eds.), p. 361. Springer-Verlag (Lecture Notes in Physics, Vol. 546), Hamburg, Germany (2000).

[31] K. Golec-Biernat and M. Wüsthoff, Phys. Rev. D 59, 014017 (1999);

K. Golec-Biernat and M. Wüsthoff, Phys. Rev. D 60, 114023 (1999);

K. Golec-Biernat and M. Wüsthoff, Eur. Phys. J. C 20, 313 (2001).

[32] H. Jung, Comput. Phys. Commun. 86, 147 (1995).

[33] G. Ingelmann and P.E. Schlein, Phys. Lett. B 152, 256 (1985).

[34] A. Donnachie and P.L. Landshoff, Phys. Lett. B 296, 227 (1992).

[35] H1 Coll., C. Adloff et al., Z. Phys. C 76, 613 (1997).

[36] J.F. Owens, Phys. Rev. D 30, 943 (1984).

[37] M. Bengtsson and T. Sjöstrand, Z. Phys. C 37, 465 (1988).

[38] L. Lönnblad, Comput. Phys. Commun. 71, 15 (1992).

[39] H-U. Bengtsson and T. Sjöstrand, Comput. Phys. Commun. 46, 43 (1987).

[40] T. Sjöstrand, Comput. Phys. Commun. 82, 74 (1994).

[41] K. Kwiatkowski, H. Spiesberger and H.-J. Möhring, Comput. Phys. Commun. 69, 155 (1992). 
[42] G.A. Schuler and H. Spiesberger, Proc. of the Workshop on HERA Physics 1991, W. Buchmüller and G. Ingelman (eds.), Vol. 3, p. 1419. DESY, Hamburg, Germany (1992).

[43] H.L. Lai et al., Phys. Rev. D 55, 1280 (1997).

[44] T. Sjöstrand, L. Lönnblad and S. Mrenna, hep-ph/0108264 (2001).

[45] R. Brun et al., Geant3, Technical Report CERN-DD/EE/84-1, CERN, 1987.

[46] UA4 Coll., D. Bernard et al., Phys. Lett. B 186, 227 (1987).

[47] ZEUS Coll., S. Chekanov et al., PMC Phys. A 1, 6 (2007).

[48] T. Gehrmann and W.J. Stirling, Z. Phys. C 70, 89 (1996).

[49] T. Arens et al., Z. Phys. C 74, 651 (1997).

[50] M. Diehl, Z. Phys. C 76, 499 (1997).

[51] N.N. Nikolaev, A.V. Pronyaev and B.G. Zakharov, Phys. Rev. D 59, 091501 (1999).

[52] J.-R. Cudell, K. Kang and S.K. Kim, Phys. Lett. B 395, 311 (1997).

[53] I.P. Ivanov, N.N. Nikolaev and A.A. Savin, hep-ph/0501034 (2005).

[54] M. Glück, E. Reya and A. Vogt, Z. Phys. C 53, 127 (1992).

[55] M. Glück, E. Reya and A. Vogt, Z. Phys. C 53, 651 (1992).

[56] M. Glück, E. Reya and A. Vogt, Z. Phys. C 67, 433 (1995). 


\begin{tabular}{|c|c|c|}
\hline$x_{\mathbb{P P}}$ & $|t|\left(\mathrm{GeV}^{2}\right)$ & $\begin{array}{c}d \sigma^{e p \rightarrow e X p} / d t\left(\mathrm{nb} / \mathrm{GeV}^{-2}\right) \\
\pm \text { stat. } \pm \text { syst. }\end{array}$ \\
\hline $0.0002-0.01$ & 0.144 & $63.59 \pm 1.33_{-1.40}^{+1.52}$ \\
$0.0002-0.01$ & 0.259 & $25.79 \pm 1.00_{-1.15}^{+1.10}$ \\
$0.0002-0.01$ & 0.374 & $12.85 \pm 0.66_{-0.89}^{+0.74}$ \\
$0.0002-0.01$ & 0.489 & $6.53 \pm 0.54_{-0.71}^{+0.71}$ \\
$0.01-0.1$ & 0.144 & $84.74 \pm 1.49_{-1.80}^{+1.81}$ \\
$0.01-0.1$ & 0.259 & $35.95 \pm 1.09_{-1.72}^{+1.70}$ \\
$0.01-0.1$ & 0.374 & $16.73 \pm 0.66_{-1.25}^{+1.16}$ \\
$0.01-0.1$ & 0.489 & $8.84 \pm 0.53_{-0.99}^{+0.96}$ \\
\hline
\end{tabular}

Table 1: The differential cross-section $d \sigma^{e p \rightarrow e X p} / d t$ as a function of $|t|$, for the indicated $x_{I P}$ ranges and for $2<Q^{2}<120 \mathrm{GeV}^{2}\left(\left\langle Q^{2}\right\rangle=11 \mathrm{GeV}^{2}\right)$ and $2<M_{X}<40 \mathrm{GeV}\left(\left\langle M_{X}\right\rangle=15 \mathrm{GeV}\right)$. 


\begin{tabular}{|c|c|c|c|}
\hline$Q^{2}\left(\mathrm{GeV}^{2}\right)$ & $M_{X}(\mathrm{GeV})$ & $x_{\mathbb{P}}$ & $b\left(\mathrm{GeV}^{-2}\right) \pm$ stat. \pm syst. \\
\hline $2-5$ & $2-5$ & 0.0004 & $6.99 \pm 0.58_{-0.28}^{+0.50}$ \\
$2-5$ & $2-5$ & 0.0018 & $5.57 \pm 0.56_{-0.20}^{+0.35}$ \\
$2-5$ & $2-5$ & 0.0060 & $6.46 \pm 0.82_{-0.37}^{+0.39}$ \\
$2-5$ & $5-10$ & 0.0018 & $6.84 \pm 0.71_{-0.39}^{+0.30}$ \\
$2-5$ & $5-10$ & 0.0060 & $6.23 \pm 0.78_{-0.48}^{+0.48}$ \\
$2-5$ & $5-10$ & 0.0200 & $7.08 \pm 0.87_{-0.69}^{+0.23}$ \\
$2-5$ & $10-40$ & 0.0060 & $8.55 \pm 0.77_{-0.70}^{+0.47}$ \\
$2-5$ & $10-40$ & 0.0200 & $7.13 \pm 0.69_{-0.36}^{+0.31}$ \\
$2-5$ & $10-40$ & 0.0400 & $7.92 \pm 0.78_{-0.25}^{+0.45}$ \\
$2-5$ & $10-40$ & 0.0600 & $6.77 \pm 0.61_{-0.46}^{+0.07}$ \\
$2-5$ & $10-40$ & 0.0850 & $6.59 \pm 0.48_{-0.17}^{+0.11}$ \\
$5-20$ & $2-5$ & 0.0004 & $7.77 \pm 0.58_{-0.69}^{+0.13}$ \\
$5-20$ & $2-5$ & 0.0018 & $7.42 \pm 0.59_{-0.21}^{+0.38}$ \\
$5-20$ & $2-5$ & 0.0060 & $8.49 \pm 0.70_{-0.55}^{+0.33}$ \\
$5-20$ & $2-5$ & 0.0200 & $7.65 \pm 1.28_{-0.42}^{+0.44}$ \\
$5-20$ & $5-10$ & 0.0018 & $7.35 \pm 0.71_{-0.27}^{+0.23}$ \\
$5-20$ & $5-10$ & 0.0060 & $6.68 \pm 0.71_{-0.37}^{+0.13}$ \\
$5-20$ & $5-10$ & 0.0200 & $6.78 \pm 0.74_{-0.07}^{+0.46}$ \\
$5-20$ & $5-10$ & 0.0400 & $11.28 \pm 1.57_{-1.70}^{+0.41}$ \\
$5-20$ & $10-40$ & 0.0060 & $7.08 \pm 0.71_{-0.29}^{+0.11}$ \\
$5-20$ & $10-40$ & 0.0200 & $6.35 \pm 0.46_{-0.22}^{+0.22}$ \\
$5-20$ & $10-40$ & 0.0400 & $8.36 \pm 0.63_{-0.21}^{+0.13}$ \\
$5-20$ & $10-40$ & 0.0600 & $7.15 \pm 0.48_{-0.11}^{+0.15}$ \\
$5-20$ & $10-40$ & 0.0850 & $6.23 \pm 0.36_{-0.02}^{+0.12}$ \\
$20-120$ & $2-5$ & 0.0018 & $6.45 \pm 1.11_{-0.61}^{+0.40}$ \\
$20-120$ & $2-5$ & 0.0060 & $7.06 \pm 1.22_{-0.79}^{+0.19}$ \\
$20-120$ & $5-10$ & 0.0018 & $7.94 \pm 1.33_{-0.26}^{+0.34}$ \\
$20-120$ & $5-10$ & 0.0060 & $5.60 \pm 1.14_{-0.63}^{+0.00}$ \\
$20-120$ & $5-10$ & 0.0200 & $6.62 \pm 1.23_{-0.06}^{+0.41}$ \\
$20-120$ & $10-40$ & 0.0060 & $5.17 \pm 1.08_{-0.07}^{+0.44}$ \\
$20-120$ & $10-40$ & 0.0200 & $9.10 \pm 0.82_{-0.35}^{+0.39}$ \\
$20-120$ & $10-40$ & 0.0400 & $6.89 \pm 0.90_{-0.54}^{+0.17}$ \\
\hline-0.23 & $10-40$ & 0.0600 & $7.17 \pm 0.69_{-0.12}^{+0.23}$ \\
$20-40$ & 0.0850 & $6.17 \pm 0.51_{-0.20}^{+0.06}$ \\
\hline
\end{tabular}

Table 2: Fitted values of the exponential t-slopes in bins of $Q^{2}, M_{X}$ and $x_{\mathbb{P}}$. 


\begin{tabular}{|c|c|c|}
\hline$x_{\mathbb{P}}$ & $\Phi(\mathrm{rad})$ & $\begin{array}{c}d \sigma^{e p} / d \Phi(\mathrm{nb} / \mathrm{rad}) \\
\pm \text { stat. } \pm \text { syst. }\end{array}$ \\
\hline $0.0002-0.01$ & 0.524 & $1.03 \pm 0.07_{-0.04}^{+0.06}$ \\
$0.0002-0.01$ & 1.571 & $1.13 \pm 0.07_{-0.11}^{+0.03}$ \\
$0.0002-0.01$ & 2.618 & $1.09 \pm 0.07_{-0.11}^{+0.03}$ \\
$0.0002-0.01$ & 3.665 & $1.07 \pm 0.06_{-0.04}^{+0.06}$ \\
$0.0002-0.01$ & 4.712 & $1.06 \pm 0.07_{-0.06}^{+0.04}$ \\
$0.0002-0.01$ & 5.759 & $0.99 \pm 0.07_{-0.04}^{+0.07}$ \\
$0.01-0.1$ & 0.524 & $1.59 \pm 0.07_{-0.08}^{+0.05}$ \\
$0.01-0.1$ & 1.571 & $1.67 \pm 0.07_{-0.05}^{+0.11}$ \\
$0.01-0.1$ & 2.618 & $1.47 \pm 0.06_{-0.10}^{+0.05}$ \\
$0.01-0.1$ & 3.665 & $1.50 \pm 0.06_{-0.06}^{+0.06}$ \\
$0.01-0.1$ & 4.712 & $1.49 \pm 0.06_{-0.08}^{+0.05}$ \\
$0.01-0.1$ & 5.759 & $1.65 \pm 0.07_{-0.12}^{+0.03}$ \\
\hline
\end{tabular}

Table 3: The differential cross-section $d \sigma^{e p \rightarrow e X p} / d \Phi$ as a function of $\Phi$, for the indicated $x_{I P}$ ranges and for $4<Q^{2}<120 \mathrm{GeV}^{2}\left(\left\langle Q^{2}\right\rangle=22 \mathrm{GeV}^{2}\right)$ and $2<M_{X}<40 \mathrm{GeV}\left(\left\langle M_{X}\right\rangle=15 \mathrm{GeV}\right)$. 


\begin{tabular}{|c|c|c|c|c|}
\hline$Q^{2}\left(\mathrm{GeV}^{2}\right)$ & $\beta$ & $|t|\left(\mathrm{GeV}^{2}\right)$ & $x_{\mathbb{P}}$ & $A_{L T} \pm$ stat. \pm syst. \\
\hline $4-120$ & $0-1$ & $0.09-0.55$ & $0.0002-0.0018$ & $0.05 \pm 0.07_{-0.02}^{+0.06}$ \\
$4-120$ & $0-1$ & $0.09-0.55$ & $0.0018-0.0042$ & $0.02 \pm 0.08_{-0.07}^{+0.02}$ \\
$4-120$ & $0-1$ & $0.09-0.55$ & $0.0042-0.01$ & $-0.06 \pm 0.08_{-0.02}^{+0.04}$ \\
$4-120$ & $0-1$ & $0.09-0.55$ & $0.01-0.03$ & $0.08 \pm 0.06_{-0.02}^{+0.04}$ \\
$4-120$ & $0-1$ & $0.09-0.55$ & $0.03-0.05$ & $0.06 \pm 0.08_{-0.02}^{+0.02}$ \\
$4-120$ & $0-1$ & $0.09-0.55$ & $0.05-0.07$ & $0.17 \pm 0.07_{-0.02}^{+0.02}$ \\
$4-120$ & $0-1$ & $0.09-0.55$ & $0.07-0.1$ & $0.05 \pm 0.05_{-0.01}^{+0.02}$ \\
\hline $4-120$ & $0-0.2$ & $0.09-0.55$ & $0.0002-0.01$ & $-0.09 \pm 0.07_{-0.03}^{+0.04}$ \\
$4-120$ & $0.2-0.35$ & $0.09-0.55$ & $0.0002-0.01$ & $0.09 \pm 0.09_{-0.07}^{+0.05}$ \\
$4-120$ & $0.35-0.65$ & $0.09-0.55$ & $0.0002-0.01$ & $0.03 \pm 0.07_{-0.01}^{+0.03}$ \\
$4-120$ & $0.65-1.0$ & $0.09-0.55$ & $0.0002-0.01$ & $0.03 \pm 0.12_{-0.04}^{+0.04}$ \\
\hline $4-120$ & $0-1$ & $0.09-0.14$ & $0.0002-0.01$ & $0.01 \pm 0.06_{-0.01}^{+0.03}$ \\
$4-120$ & $0-1$ & $0.14-0.2$ & $0.0002-0.01$ & $0.12 \pm 0.11_{-0.02}^{+0.06}$ \\
$4-120$ & $0-1$ & $0.2-0.3$ & $0.0002-0.01$ & $-0.05 \pm 0.10_{-0.05}^{+0.02}$ \\
$4-120$ & $0-1$ & $0.3-0.55$ & $0.0002-0.01$ & $-0.05 \pm 0.10_{-0.02}^{+0.04}$ \\
\hline $4-10$ & $0-1$ & $0.09-0.55$ & $0.0002-0.01$ & $0.04 \pm 0.07_{-0.02}^{+0.07}$ \\
$10-15$ & $0-1$ & $0.09-0.55$ & $0.0002-0.01$ & $-0.05 \pm 0.08_{-0.02}^{+0.03}$ \\
$15-30$ & $0-1$ & $0.09-0.55$ & $0.0002-0.01$ & $0.10 \pm 0.08_{-0.04}^{+0.02}$ \\
$30-120$ & $0-1$ & $0.09-0.55$ & $0.0002-0.01$ & $-0.23 \pm 0.12_{-0.05}^{+0.02}$ \\
\hline
\end{tabular}

Table 4: The azimuthal asymmetry $A_{L T}$ in bins of $Q^{2}, \beta,|t|$ and $x_{\mathbb{P}}$. 


\begin{tabular}{|c|c|c|c|c|}
\hline$Q^{2}\left(\mathrm{GeV}^{2}\right)$ & $\beta$ & $|t|\left(\mathrm{GeV}^{2}\right)$ & $x_{\mathbb{P}}$ & $A_{T T} \pm$ stat. \pm syst. \\
\hline $4-120$ & $0-1$ & $0.09-0.55$ & $0.0002-0.0018$ & $0.01 \pm 0.07_{-0.03}^{+0.07}$ \\
$4-120$ & $0-1$ & $0.09-0.55$ & $0.0018-0.0042$ & $-0.01 \pm 0.08_{-0.04}^{+0.05}$ \\
$4-120$ & $0-1$ & $0.09-0.55$ & $0.0042-0.01$ & $-0.17 \pm 0.08_{-0.02}^{+0.06}$ \\
$4-120$ & $0-1$ & $0.09-0.55$ & $0.01-0.03$ & $0.05 \pm 0.06_{-0.02}^{+0.03}$ \\
$4-120$ & $0-1$ & $0.09-0.55$ & $0.03-0.05$ & $-0.11 \pm 0.08_{-0.06}^{+0.03}$ \\
$4-120$ & $0-1$ & $0.09-0.55$ & $0.05-0.07$ & $0.06 \pm 0.07_{-0.04}^{+0.03}$ \\
$4-120$ & $0-1$ & $0.09-0.55$ & $0.07-0.01$ & $0.02 \pm 0.05_{-0.01}^{+0.02}$ \\
\hline $4-120$ & $0-0.2$ & $0.09-0.55$ & $0.0002-0.01$ & $-0.14 \pm 0.07_{-0.00}^{+0.07}$ \\
$4-120$ & $0.2-0.35$ & $0.09-0.55$ & $0.0002-0.01$ & $0.05 \pm 0.09_{-0.09}^{+0.03}$ \\
$4-120$ & $0.35-0.65$ & $0.09-0.55$ & $0.0002-0.01$ & $-0.01 \pm 0.07_{-0.01}^{+0.04}$ \\
$4-120$ & $0.65-1.0$ & $0.09-0.55$ & $0.0002-0.01$ & $-0.16 \pm 0.12_{-0.01}^{+0.08}$ \\
\hline $4-120$ & $0-1$ & $0.09-0.14$ & $0.0002-0.01$ & $-0.10 \pm 0.06_{-0.02}^{+0.03}$ \\
$4-120$ & $0-1$ & $0.14-0.2$ & $0.0002-0.01$ & $0.13 \pm 0.11_{-0.07}^{+0.05}$ \\
$4-120$ & $0-1$ & $0.2-0.3$ & $0.0002-0.01$ & $-0.01 \pm 0.10_{-0.05}^{+0.05}$ \\
$4-120$ & $0-1$ & $0.3-0.55$ & $0.0002-0.01$ & $-0.11 \pm 0.10_{-0.01}^{+0.16}$ \\
\hline $4-10$ & $0-1$ & $0.09-0.55$ & $0.0002-0.01$ & $-0.01 \pm 0.07_{-0.04}^{+0.11}$ \\
$10-15$ & $0-1$ & $0.09-0.55$ & $0.0002-0.01$ & $-0.12 \pm 0.08_{-0.02}^{+0.04}$ \\
$15-30$ & $0-1$ & $0.09-0.55$ & $0.0002-0.01$ & $-0.03 \pm 0.08_{-0.02}^{+0.04}$ \\
$30-120$ & $0-1$ & $0.09-0.55$ & $0.0002-0.01$ & $-0.12 \pm 0.12_{-0.04}^{+0.05}$ \\
\hline
\end{tabular}

Table 5: The azimuthal asymmetry $A_{T T}$ in bins of $Q^{2}, \beta,|t|$ and $x_{\mathbb{P}}$. 


\begin{tabular}{|c|c|c|c|c|}
\hline$Q^{2}\left(\mathrm{GeV}^{2}\right)$ & $M_{X}(\mathrm{GeV})$ & $\beta$ & $x_{\mathbb{P}}$ & $\begin{array}{c}x_{\mathbb{P}} \int \sigma_{r}^{D(4)} d t / \Delta t\left(\mathrm{GeV}^{-2}\right) \\
\pm \text { stat. } \pm \text { syst. }\end{array}$ \\
\hline 2.5 & 3 & 0.217 & 0.0003 & $0.102 \pm 0.012_{-0.003}^{+0.009}$ \\
2.5 & 3 & 0.217 & 0.0009 & $0.069 \pm 0.009_{-0.002}^{+0.004}$ \\
2.5 & 3 & 0.217 & 0.0025 & $0.036 \pm 0.006_{-0.004}^{+0.007}$ \\
2.5 & 3 & 0.217 & 0.0065 & $0.044 \pm 0.009_{-0.004}^{+0.004}$ \\
2.5 & 7 & 0.049 & 0.0009 & $0.064 \pm 0.010_{-0.007}^{+0.004}$ \\
2.5 & 7 & 0.049 & 0.0025 & $0.052 \pm 0.007_{-0.006}^{+0.002}$ \\
2.5 & 7 & 0.049 & 0.0065 & $0.036 \pm 0.005_{-0.001}^{+0.004}$ \\
2.5 & 7 & 0.049 & 0.0150 & $0.044 \pm 0.008_{-0.005}^{+0.004}$ \\
2.5 & 7 & 0.049 & 0.0300 & $0.039 \pm 0.009_{-0.005}^{+0.006}$ \\
2.5 & 15 & 0.011 & 0.0065 & $0.044 \pm 0.008_{-0.002}^{+0.007}$ \\
2.5 & 15 & 0.011 & 0.0150 & $0.035 \pm 0.007_{-0.004}^{+0.013}$ \\
2.5 & 15 & 0.011 & 0.0300 & $0.063 \pm 0.011_{-0.005}^{+0.005}$ \\
2.5 & 15 & 0.011 & 0.0500 & $0.064 \pm 0.014_{-0.010}^{+0.012}$ \\
2.5 & 15 & 0.011 & 0.0700 & $0.093 \pm 0.017_{-0.004}^{+0.006}$ \\
2.5 & 15 & 0.011 & 0.0900 & $0.081 \pm 0.015_{-0.012}^{+0.016}$ \\
2.5 & 30 & 0.003 & 0.0300 & $0.063 \pm 0.010_{-0.006}^{+0.005}$ \\
2.5 & 30 & 0.003 & 0.0500 & $0.052 \pm 0.012_{-0.003}^{+0.008}$ \\
2.5 & 30 & 0.003 & 0.0700 & $0.058 \pm 0.010_{-0.004}^{+0.001}$ \\
2.5 & 30 & 0.003 & 0.0900 & $0.077 \pm 0.012_{-0.018}^{+0.004}$ \\
3.9 & 3 & 0.302 & 0.0003 & $0.089 \pm 0.010_{-0.004}^{+0.005}$ \\
3.9 & 3 & 0.302 & 0.0009 & $0.064 \pm 0.008_{-0.004}^{+0.008}$ \\
3.9 & 3 & 0.302 & 0.0025 & $0.060 \pm 0.008_{-0.002}^{+0.005}$ \\
3.9 & 3 & 0.302 & 0.0065 & $0.055 \pm 0.009_{-0.003}^{+0.003}$ \\
3.9 & 7 & 0.074 & 0.0025 & $0.051 \pm 0.006_{-0.003}^{+0.004}$ \\
3.9 & 7 & 0.074 & 0.0065 & $0.047 \pm 0.006_{-0.005}^{+0.001}$ \\
3.9 & 7 & 0.074 & 0.0150 & $0.041 \pm 0.007_{-0.003}^{+0.002}$ \\
3.9 & 7 & 0.074 & 0.0300 & $0.054 \pm 0.011_{-0.008}^{+0.010}$ \\
\hline
\end{tabular}

Table 6: The reduced diffractive cross section multiplied by $x_{\mathbb{P}}, x_{\mathbb{P}} \sigma_{r}^{D(4)}$, obtained with the LPS method for different values of $Q^{2}, M_{X}$ and $x_{\mathbb{P}}$ and for $0.09<|t|<$ $0.19 \mathrm{GeV}^{2}\left(\langle|t|\rangle=0.13 \mathrm{GeV}^{2}\right)$. The corresponding $\beta$ values are also indicated. The table continues on the next 2 pages. 


\begin{tabular}{|c|c|c|c|c|}
\hline$Q^{2}\left(\mathrm{GeV}^{2}\right)$ & $M_{X}(\mathrm{GeV})$ & $\beta$ & $x_{\mathbb{P}}$ & $\begin{array}{c}x_{\mathbb{P}} \int \sigma_{r}^{D(4)} d t / \Delta t\left(\mathrm{GeV}^{-2}\right) \\
\pm \text { stat. } \pm \text { syst. }\end{array}$ \\
\hline 3.9 & 15 & 0.017 & 0.0065 & $0.058 \pm 0.008_{-0.002}^{+0.004}$ \\
3.9 & 15 & 0.017 & 0.0150 & $0.054 \pm 0.008_{-0.008}^{+0.006}$ \\
3.9 & 15 & 0.017 & 0.0300 & $0.053 \pm 0.009_{-0.003}^{+0.002}$ \\
3.9 & 15 & 0.017 & 0.0500 & $0.041 \pm 0.009_{-0.002}^{+0.004}$ \\
3.9 & 15 & 0.017 & 0.0700 & $0.064 \pm 0.010_{-0.003}^{+0.003}$ \\
3.9 & 15 & 0.017 & 0.0900 & $0.068 \pm 0.010_{-0.004}^{+0.003}$ \\
3.9 & 30 & 0.004 & 0.0300 & $0.065 \pm 0.010_{-0.007}^{+0.009}$ \\
3.9 & 30 & 0.004 & 0.0500 & $0.077 \pm 0.015_{-0.012}^{+0.003}$ \\
3.9 & 30 & 0.004 & 0.0700 & $0.103 \pm 0.015_{-0.014}^{+0.004}$ \\
3.9 & 30 & 0.004 & 0.0900 & $0.092 \pm 0.013_{-0.005}^{+0.013}$ \\
7.1 & 3 & 0.441 & 0.0003 & $0.107 \pm 0.012_{-0.005}^{+0.005}$ \\
7.1 & 3 & 0.441 & 0.0009 & $0.113 \pm 0.011_{-0.010}^{+0.003}$ \\
7.1 & 3 & 0.441 & 0.0025 & $0.086 \pm 0.010_{-0.002}^{+0.006}$ \\
7.1 & 3 & 0.441 & 0.0065 & $0.082 \pm 0.010_{-0.008}^{+0.002}$ \\
7.1 & 7 & 0.127 & 0.0025 & $0.069 \pm 0.006_{-0.003}^{+0.003}$ \\
7.1 & 7 & 0.127 & 0.0065 & $0.054 \pm 0.006_{-0.004}^{+0.004}$ \\
7.1 & 7 & 0.127 & 0.0150 & $0.053 \pm 0.007_{-0.003}^{+0.005}$ \\
7.1 & 7 & 0.127 & 0.0300 & $0.074 \pm 0.011_{-0.007}^{+0.002}$ \\
7.1 & 15 & 0.031 & 0.0065 & $0.067 \pm 0.008_{-0.008}^{+0.005}$ \\
7.1 & 15 & 0.031 & 0.0150 & $0.061 \pm 0.007_{-0.005}^{+0.004}$ \\
7.1 & 15 & 0.031 & 0.0300 & $0.063 \pm 0.008_{-0.005}^{+0.004}$ \\
7.1 & 15 & 0.031 & 0.0500 & $0.105 \pm 0.015_{-0.003}^{+0.007}$ \\
7.1 & 15 & 0.031 & 0.0700 & $0.068 \pm 0.008_{-0.003}^{+0.004}$ \\
7.1 & 15 & 0.031 & 0.0900 & $0.075 \pm 0.009_{-0.003}^{+0.003}$ \\
7.1 & 30 & 0.008 & 0.0300 & $0.089 \pm 0.010_{-0.007}^{+0.006}$ \\
7.1 & 30 & 0.008 & 0.0500 & $0.104 \pm 0.014_{-0.007}^{+0.007}$ \\
7.1 & 30 & 0.008 & 0.0700 & $0.084 \pm 0.009_{-0.003}^{+0.005}$ \\
7.1 & 30 & 0.008 & 0.0900 & $0.133 \pm 0.014_{-0.011}^{+0.008}$ \\
14 & 3 & 0.609 & 0.0009 & $0.110 \pm 0.014_{-0.009}^{+0.005}$ \\
& & 0.609 & 0.0025 & $0.093 \pm 0.014_{-0.004}^{+0.007}$ \\
& 3 & 0.609 & 0.0065 & $0.102 \pm 0.016_{-0.003}^{+0.007}$ \\
\hline
\end{tabular}




\begin{tabular}{|c|c|c|c|c|}
\hline$Q^{2}\left(\mathrm{GeV}^{2}\right)$ & $M_{X}(\mathrm{GeV})$ & $\beta$ & $x_{\mathbb{P}}$ & $\begin{array}{c}x_{\mathbb{P}} \int \sigma_{r}^{D(4)} d t / \Delta t\left(\mathrm{GeV}^{-2}\right) \\
\pm \text { stat. } \pm \text { syst. }\end{array}$ \\
\hline 14 & 7 & 0.222 & 0.0025 & $0.071 \pm 0.008_{-0.006}^{+0.002}$ \\
\hline 14 & 7 & 0.222 & 0.0065 & $0.067 \pm 0.008_{-0.004}^{+0.003}$ \\
\hline 14 & 7 & 0.222 & 0.0150 & $0.074 \pm 0.010_{-0.003}^{+0.005}$ \\
\hline 14 & 7 & 0.222 & 0.0300 & $0.059 \pm 0.010_{-0.006}^{+0.002}$ \\
\hline 14 & 15 & 0.059 & 0.0065 & $0.070 \pm 0.009_{-0.004}^{+0.002}$ \\
\hline 14 & 15 & 0.059 & 0.0150 & $0.083 \pm 0.011_{-0.006}^{+0.005}$ \\
\hline 14 & 15 & 0.059 & 0.0300 & $0.065 \pm 0.009_{-0.001}^{+0.006}$ \\
\hline 14 & 15 & 0.059 & 0.0500 & $0.110 \pm 0.017_{-0.006}^{+0.007}$ \\
\hline 14 & 15 & 0.059 & 0.0700 & $0.088 \pm 0.011_{-0.004}^{+0.002}$ \\
\hline 14 & 15 & 0.059 & 0.0900 & $0.090 \pm 0.011_{-0.003}^{+0.003}$ \\
\hline 14 & 30 & 0.015 & 0.0300 & $0.097 \pm 0.013_{-0.004}^{+0.004}$ \\
\hline 14 & 30 & 0.015 & 0.0500 & $0.107 \pm 0.017_{-0.005}^{+0.006}$ \\
\hline 14 & 30 & 0.015 & 0.0700 & $0.109 \pm 0.013_{-0.003}^{+0.003}$ \\
\hline 14 & 30 & 0.015 & 0.0900 & $0.113 \pm 0.012_{-0.005}^{+0.005}$ \\
\hline 40 & 3 & 0.816 & 0.0009 & $0.094 \pm 0.024_{-0.008}^{+0.008}$ \\
\hline 40 & 3 & 0.816 & 0.0025 & $0.061 \pm 0.013_{-0.006}^{+0.008}$ \\
\hline 40 & 3 & 0.816 & 0.0065 & $0.054 \pm 0.013_{-0.005}^{+0.003}$ \\
\hline 40 & 3 & 0.816 & 0.0150 & $0.049 \pm 0.016_{-0.003}^{+0.002}$ \\
\hline 40 & 7 & 0.449 & 0.0025 & $0.104 \pm 0.013_{-0.009}^{+0.005}$ \\
\hline 40 & 7 & 0.449 & 0.0065 & $0.073 \pm 0.010_{-0.005}^{+0.003}$ \\
\hline 40 & 7 & 0.449 & 0.0150 & $0.075 \pm 0.012_{-0.002}^{+0.004}$ \\
\hline 40 & 7 & 0.449 & 0.0300 & $0.079 \pm 0.014_{-0.002}^{+0.008}$ \\
\hline 40 & 7 & 0.449 & 0.0500 & $0.081 \pm 0.023_{-0.002}^{+0.012}$ \\
\hline 40 & 15 & 0.151 & 0.0065 & $0.058 \pm 0.009_{-0.005}^{+0.005}$ \\
\hline 40 & 15 & 0.151 & 0.0150 & $0.090 \pm 0.013_{-0.004}^{+0.004}$ \\
\hline 40 & 15 & 0.151 & 0.0300 & $0.073 \pm 0.011_{-0.004}^{+0.003}$ \\
\hline 40 & 15 & 0.151 & 0.0500 & $0.093 \pm 0.016_{-0.007}^{+0.002}$ \\
\hline 40 & 15 & 0.151 & 0.0700 & $0.098 \pm 0.013_{-0.006}^{+0.004}$ \\
\hline 40 & 15 & 0.151 & 0.0900 & $0.115 \pm 0.013_{-0.005}^{+0.005}$ \\
\hline 40 & 30 & 0.043 & 0.0300 & $0.090 \pm 0.012_{-0.002}^{+0.007}$ \\
\hline 40 & 30 & 0.043 & 0.0500 & $0.127 \pm 0.018_{-0.008}^{+0.004}$ \\
\hline 40 & 30 & 0.043 & 0.0700 & $0.113 \pm 0.013_{-0.007}^{+0.003}$ \\
\hline 40 & 30 & 0.043 & 0.0900 & $0.124 \pm 0.012_{-0.005}^{+0.006}$ \\
\hline
\end{tabular}




\begin{tabular}{|c|c|c|c|c|}
\hline$Q^{2}\left(\mathrm{GeV}^{2}\right)$ & $M_{X}(\mathrm{GeV})$ & $\beta$ & $x_{I P}$ & $\begin{array}{c}x_{I P} \int \sigma_{r}^{D(4)} d t / \Delta t\left(\mathrm{GeV}^{-2}\right) \\
\pm \text { stat. } \pm \text { syst. }\end{array}$ \\
\hline 2.5 & 3 & 0.217 & 0.0003 & $0.028 \pm 0.004_{-0.004}^{+0.001}$ \\
2.5 & 3 & 0.217 & 0.0009 & $0.013 \pm 0.002_{-0.003}^{+0.003}$ \\
2.5 & 3 & 0.217 & 0.0025 & $0.013 \pm 0.002_{-0.002}^{+0.000}$ \\
2.5 & 3 & 0.217 & 0.0065 & $0.012 \pm 0.003_{-0.002}^{+0.001}$ \\
2.5 & 7 & 0.049 & 0.0009 & $0.025 \pm 0.005_{-0.003}^{+0.001}$ \\
2.5 & 7 & 0.049 & 0.0025 & $0.012 \pm 0.002_{-0.001}^{+0.001}$ \\
2.5 & 7 & 0.049 & 0.0065 & $0.009 \pm 0.002_{-0.002}^{+0.001}$ \\
2.5 & 7 & 0.049 & 0.0150 & $0.010 \pm 0.002_{-0.001}^{+0.002}$ \\
2.5 & 7 & 0.049 & 0.0300 & $0.012 \pm 0.003_{-0.002}^{+0.001}$ \\
2.5 & 15 & 0.011 & 0.0065 & $0.011 \pm 0.002_{-0.002}^{+0.001}$ \\
2.5 & 15 & 0.011 & 0.0150 & $0.011 \pm 0.002_{-0.002}^{+0.002}$ \\
2.5 & 15 & 0.011 & 0.0300 & $0.012 \pm 0.003_{-0.001}^{+0.001}$ \\
2.5 & 15 & 0.011 & 0.0500 & $0.014 \pm 0.003_{-0.001}^{+0.004}$ \\
2.5 & 15 & 0.011 & 0.0700 & $0.012 \pm 0.002_{-0.001}^{+0.002}$ \\
2.5 & 15 & 0.011 & 0.0900 & $0.023 \pm 0.005_{-0.003}^{+0.004}$ \\
2.5 & 30 & 0.003 & 0.0300 & $0.015 \pm 0.003_{-0.002}^{+0.002}$ \\
2.5 & 30 & 0.003 & 0.0500 & $0.016 \pm 0.003_{-0.003}^{+0.001}$ \\
2.5 & 30 & 0.003 & 0.0700 & $0.024 \pm 0.004_{-0.003}^{+0.002}$ \\
2.5 & 30 & 0.003 & 0.0900 & $0.015 \pm 0.003_{-0.003}^{+0.003}$ \\
3.9 & 3 & 0.302 & 0.0003 & $0.023 \pm 0.003_{-0.002}^{+0.002}$ \\
3.9 & 3 & 0.302 & 0.0009 & $0.021 \pm 0.003_{-0.002}^{+0.002}$ \\
3.9 & 3 & 0.302 & 0.0025 & $0.016 \pm 0.003_{-0.001}^{+0.002}$ \\
3.9 & 3 & 0.302 & 0.0065 & $0.010 \pm 0.002_{-0.002}^{+0.001}$ \\
3.9 & 7 & 0.074 & 0.0025 & $0.012 \pm 0.002_{-0.001}^{+0.002}$ \\
3.9 & 7 & 0.074 & 0.0065 & $0.016 \pm 0.002_{-0.002}^{+0.001}$ \\
3.9 & 7 & 0.074 & 0.0150 & $0.009 \pm 0.002_{-0.001}^{+0.002}$ \\
3.9 & 7 & 0.074 & 0.0300 & $0.013 \pm 0.003_{-0.001}^{+0.002}$ \\
\hline
\end{tabular}

Table 7: The reduced diffractive cross section multiplied by $x_{\mathbb{P}}, x_{\mathbb{P}} \sigma_{r}^{D(4)}$, obtained with the LPS method for different values of $Q^{2}, M_{X}$ and $x_{\mathbb{P}}$ and for $0.19<|t|<$ $0.55 \mathrm{GeV}^{2}\left(\langle|t|\rangle=0.3 \mathrm{GeV}^{2}\right)$. The corresponding $\beta$ values are also indicated. The table continues on the next 2 pages. 


\begin{tabular}{|c|c|c|c|c|}
\hline$Q^{2}\left(\mathrm{GeV}^{2}\right)$ & $M_{X}(\mathrm{GeV})$ & $\beta$ & $x_{\mathbb{P}}$ & $\begin{array}{c}x_{\mathbb{P}} \int \sigma_{r}^{D(4)} d t / \Delta t\left(\mathrm{GeV}^{-2}\right) \\
\pm \text { stat. } \pm \text { syst. }\end{array}$ \\
\hline 3.9 & 15 & 0.017 & 0.0065 & $0.012 \pm 0.002_{-0.001}^{+0.001}$ \\
3.9 & 15 & 0.017 & 0.0150 & $0.009 \pm 0.002_{-0.001}^{+0.001}$ \\
3.9 & 15 & 0.017 & 0.0300 & $0.011 \pm 0.002_{-0.002}^{+0.001}$ \\
3.9 & 15 & 0.017 & 0.0500 & $0.014 \pm 0.003_{-0.001}^{+0.002}$ \\
3.9 & 15 & 0.017 & 0.0700 & $0.017 \pm 0.002_{-0.002}^{+0.002}$ \\
3.9 & 15 & 0.017 & 0.0900 & $0.023 \pm 0.004_{-0.002}^{+0.002}$ \\
3.9 & 30 & 0.004 & 0.0300 & $0.015 \pm 0.003_{-0.002}^{+0.001}$ \\
3.9 & 30 & 0.004 & 0.0500 & $0.018 \pm 0.003_{-0.001}^{+0.003}$ \\
3.9 & 30 & 0.004 & 0.0700 & $0.021 \pm 0.003_{-0.002}^{+0.003}$ \\
3.9 & 30 & 0.004 & 0.0900 & $0.028 \pm 0.004_{-0.002}^{+0.002}$ \\
7.1 & 3 & 0.441 & 0.0003 & $0.034 \pm 0.005_{-0.003}^{+0.003}$ \\
7.1 & 3 & 0.441 & 0.0009 & $0.022 \pm 0.003_{-0.001}^{+0.002}$ \\
7.1 & 3 & 0.441 & 0.0025 & $0.019 \pm 0.003_{-0.002}^{+0.002}$ \\
7.1 & 3 & 0.441 & 0.0065 & $0.015 \pm 0.003_{-0.002}^{+0.002}$ \\
7.1 & 7 & 0.127 & 0.0025 & $0.014 \pm 0.002_{-0.001}^{+0.001}$ \\
7.1 & 7 & 0.127 & 0.0065 & $0.011 \pm 0.002_{-0.001}^{+0.001}$ \\
7.1 & 7 & 0.127 & 0.0150 & $0.013 \pm 0.002_{-0.002}^{+0.001}$ \\
7.1 & 7 & 0.127 & 0.0300 & $0.011 \pm 0.002_{-0.001}^{+0.002}$ \\
7.1 & 15 & 0.031 & 0.0065 & $0.019 \pm 0.003_{-0.001}^{+0.001}$ \\
7.1 & 15 & 0.031 & 0.0150 & $0.016 \pm 0.002_{-0.002}^{+0.003}$ \\
7.1 & 15 & 0.031 & 0.0300 & $0.018 \pm 0.003_{-0.002}^{+0.001}$ \\
7.1 & 15 & 0.031 & 0.0500 & $0.018 \pm 0.003_{-0.001}^{+0.003}$ \\
7.1 & 15 & 0.031 & 0.0700 & $0.024 \pm 0.003_{-0.002}^{+0.002}$ \\
7.1 & 15 & 0.031 & 0.0900 & $0.028 \pm 0.004_{-0.003}^{+0.002}$ \\
7.1 & 30 & 0.008 & 0.0300 & $0.018 \pm 0.003_{-0.001}^{+0.001}$ \\
7.1 & 30 & 0.008 & 0.0500 & $0.021 \pm 0.003_{-0.002}^{+0.001}$ \\
7.1 & 30 & 0.008 & 0.0700 & $0.025 \pm 0.003_{-0.002}^{+0.002}$ \\
7.1 & 30 & 0.008 & 0.0900 & $0.031 \pm 0.004_{-0.002}^{+0.002}$ \\
14 & 3 & 0.609 & 0.0009 & $0.018 \pm 0.004_{-0.003}^{+0.002}$ \\
& & 0.609 & 0.0025 & $0.018 \pm 0.004_{-0.001}^{+0.002}$ \\
-0.002 \\
\hline
\end{tabular}




\begin{tabular}{|c|c|c|c|c|}
\hline$Q^{2}\left(\mathrm{GeV}^{2}\right)$ & $M_{X}(\mathrm{GeV})$ & $\beta$ & $x_{\mathbb{P}}$ & $\begin{array}{c}x_{\mathbb{P}} \int \sigma_{r}^{D(4)} d t / \Delta t\left(\mathrm{GeV}^{-2}\right) \\
\pm \text { stat. } \pm \text { syst. }\end{array}$ \\
\hline 14 & 7 & 0.222 & 0.0025 & $0.020 \pm 0.003_{-0.002}^{+0.001}$ \\
\hline 14 & 7 & 0.222 & 0.0065 & $0.015 \pm 0.002_{-0.001}^{+0.001}$ \\
\hline 14 & 7 & 0.222 & 0.0150 & $0.016 \pm 0.003_{-0.001}^{+0.001}$ \\
\hline 14 & 7 & 0.222 & 0.0300 & $0.018 \pm 0.003_{-0.001}^{+0.001}$ \\
\hline 14 & 15 & 0.059 & 0.0065 & $0.015 \pm 0.003_{-0.002}^{+0.001}$ \\
\hline 14 & 15 & 0.059 & 0.0150 & $0.021 \pm 0.003_{-0.001}^{+0.001}$ \\
\hline 14 & 15 & 0.059 & 0.0300 & $0.022 \pm 0.004_{-0.003}^{+0.002}$ \\
\hline 14 & 15 & 0.059 & 0.0500 & $0.021 \pm 0.004_{-0.001}^{+0.002}$ \\
\hline 14 & 15 & 0.059 & 0.0700 & $0.024 \pm 0.003_{-0.002}^{+0.002}$ \\
\hline 14 & 15 & 0.059 & 0.0900 & $0.019 \pm 0.003_{-0.002}^{+0.002}$ \\
\hline 14 & 30 & 0.015 & 0.0300 & $0.020 \pm 0.003_{-0.002}^{+0.001}$ \\
\hline 14 & 30 & 0.015 & 0.0500 & $0.018 \pm 0.003_{-0.001}^{+0.001}$ \\
\hline 14 & 30 & 0.015 & 0.0700 & $0.025 \pm 0.003_{-0.002}^{+0.002}$ \\
\hline 14 & 30 & 0.015 & 0.0900 & $0.034 \pm 0.004_{-0.004}^{+0.002}$ \\
\hline 40 & 3 & 0.816 & 0.0009 & $0.018 \pm 0.006_{-0.003}^{+0.001}$ \\
\hline 40 & 3 & 0.816 & 0.0025 & $0.023 \pm 0.006_{-0.002}^{+0.002}$ \\
\hline 40 & 3 & 0.816 & 0.0065 & $0.019 \pm 0.005_{-0.002}^{+0.002}$ \\
\hline 40 & 3 & 0.816 & 0.0150 & $0.012 \pm 0.006_{-0.003}^{+0.003}$ \\
\hline 40 & 7 & 0.449 & 0.0025 & $0.023 \pm 0.004_{-0.001}^{+0.001}$ \\
\hline 40 & 7 & 0.449 & 0.0065 & $0.022 \pm 0.003_{-0.001}^{+0.002}$ \\
\hline 40 & 7 & 0.449 & 0.0150 & $0.017 \pm 0.003_{-0.001}^{+0.001}$ \\
\hline 40 & 7 & 0.449 & 0.0300 & $0.014 \pm 0.003_{-0.001}^{+0.001}$ \\
\hline 40 & 7 & 0.449 & 0.0500 & $0.019 \pm 0.006_{-0.004}^{+0.002}$ \\
\hline 40 & 15 & 0.151 & 0.0065 & $0.022 \pm 0.004_{-0.002}^{+0.001}$ \\
\hline 40 & 15 & 0.151 & 0.0150 & $0.016 \pm 0.003_{-0.002}^{+0.001}$ \\
\hline 40 & 15 & 0.151 & 0.0300 & $0.013 \pm 0.003_{-0.001}^{+0.002}$ \\
\hline 40 & 15 & 0.151 & 0.0500 & $0.025 \pm 0.004_{-0.002}^{+0.002}$ \\
\hline 40 & 15 & 0.151 & 0.0700 & $0.022 \pm 0.003_{-0.002}^{+0.002}$ \\
\hline 40 & 15 & 0.151 & 0.0900 & $0.027 \pm 0.004_{-0.002}^{+0.003}$ \\
\hline 40 & 30 & 0.043 & 0.0300 & $0.022 \pm 0.004_{-0.003}^{+0.001}$ \\
\hline 40 & 30 & 0.043 & 0.0500 & $0.028 \pm 0.004_{-0.001}^{+0.002}$ \\
\hline 40 & 30 & 0.043 & 0.0700 & $0.029 \pm 0.003_{-0.002}^{+0.002}$ \\
\hline 40 & 30 & 0.043 & 0.0900 & $0.043 \pm 0.005_{-0.004}^{+0.003}$ \\
\hline
\end{tabular}




\begin{tabular}{|c|c|c|c|c|}
\hline$Q^{2}\left(\mathrm{GeV}^{2}\right)$ & $M_{X}(\mathrm{GeV})$ & $\beta$ & $x_{I P}$ & $\begin{array}{c}x_{I P} \sigma_{r}^{D(3)} \\
\pm \text { stat. } \pm \text { syst. }\end{array}$ \\
\hline 2.5 & 3 & 0.217 & 0.0003 & $0.039 \pm 0.004_{-0.003}^{+0.003}$ \\
2.5 & 3 & 0.217 & 0.0009 & $0.023 \pm 0.002_{-0.002}^{+0.001}$ \\
2.5 & 3 & 0.217 & 0.0025 & $0.014 \pm 0.002_{-0.001}^{+0.002}$ \\
2.5 & 3 & 0.217 & 0.0065 & $0.018 \pm 0.003_{-0.005}^{+0.002}$ \\
2.5 & 6 & 0.065 & 0.0009 & $0.025 \pm 0.003_{-0.002}^{+0.003}$ \\
2.5 & 6 & 0.065 & 0.0025 & $0.016 \pm 0.002_{-0.001}^{+0.003}$ \\
2.5 & 6 & 0.065 & 0.0065 & $0.014 \pm 0.002_{-0.002}^{+0.001}$ \\
2.5 & 6 & 0.065 & 0.0150 & $0.016 \pm 0.003_{-0.002}^{+0.002}$ \\
2.5 & 11 & 0.020 & 0.0025 & $0.023 \pm 0.004_{-0.002}^{+0.002}$ \\
2.5 & 11 & 0.020 & 0.0065 & $0.015 \pm 0.002_{-0.002}^{+0.002}$ \\
2.5 & 11 & 0.020 & 0.0150 & $0.013 \pm 0.002_{-0.002}^{+0.002}$ \\
2.5 & 11 & 0.020 & 0.0300 & $0.016 \pm 0.002_{-0.002}^{+0.001}$ \\
2.5 & 11 & 0.020 & 0.0500 & $0.024 \pm 0.005_{-0.001}^{+0.004}$ \\
2.5 & 11 & 0.020 & 0.0700 & $0.023 \pm 0.004_{-0.002}^{+0.003}$ \\
2.5 & 19 & 0.007 & 0.0065 & $0.021 \pm 0.004_{-0.004}^{+0.003}$ \\
2.5 & 19 & 0.007 & 0.0150 & $0.019 \pm 0.003_{-0.003}^{+0.001}$ \\
2.5 & 19 & 0.007 & 0.0300 & $0.020 \pm 0.003_{-0.003}^{+0.002}$ \\
2.5 & 19 & 0.007 & 0.0500 & $0.019 \pm 0.003_{-0.002}^{+0.001}$ \\
2.5 & 19 & 0.007 & 0.0700 & $0.027 \pm 0.004_{-0.005}^{+0.002}$ \\
2.5 & 19 & 0.007 & 0.0900 & $0.028 \pm 0.004_{-0.003}^{+0.002}$ \\
2.5 & 32 & 0.002 & 0.0300 & $0.020 \pm 0.003_{-0.001}^{+0.003}$ \\
2.5 & 32 & 0.002 & 0.0500 & $0.020 \pm 0.004_{-0.002}^{+0.001}$ \\
2.5 & 32 & 0.002 & 0.0700 & $0.029 \pm 0.004_{-0.001}^{+0.004}$ \\
2.5 & 32 & 0.002 & 0.0900 & $0.023 \pm 0.003_{-0.002}^{+0.003}$ \\
3.9 & 3 & 0.302 & 0.0003 & $0.035 \pm 0.003_{-0.003}^{+0.002}$ \\
3.9 & 3 & 0.302 & 0.0009 & $0.026 \pm 0.002_{-0.001}^{+0.002}$ \\
3.9 & 3 & 0.302 & 0.0025 & $0.024 \pm 0.003_{-0.003}^{+0.002}$ \\
3.9 & 0.302 & 0.0065 & $0.017 \pm 0.002_{-0.002}^{+0.002}$ \\
\hline
\end{tabular}

Table 8: The reduced diffractive cross section multiplied by $x_{\mathbb{P}}, x_{\mathbb{P}} \sigma_{r}^{D(3)}$, obtained with the LPS method for different values of $Q^{2}, M_{X}$ and $x_{\mathbb{P}}$. The corresponding $\beta$ values are also indicated. The table continues on the next 3 pages. 


\begin{tabular}{|c|c|c|c|c|}
\hline$Q^{2}\left(\mathrm{GeV}^{2}\right)$ & $M_{X}(\mathrm{GeV})$ & $\beta$ & $x_{I P}$ & $\begin{array}{c}x_{\mathbb{P}} \sigma_{r}^{D(3)} \\
\pm \text { stat. } \pm \text { syst. }\end{array}$ \\
\hline 3.9 & 6 & 0.098 & 0.0009 & $0.024 \pm 0.002_{-0.001}^{+0.003}$ \\
3.9 & 6 & 0.098 & 0.0025 & $0.019 \pm 0.002_{-0.001}^{+0.001}$ \\
3.9 & 6 & 0.098 & 0.0065 & $0.017 \pm 0.002_{-0.001}^{+0.001}$ \\
3.9 & 6 & 0.098 & 0.0150 & $0.015 \pm 0.002_{-0.001}^{+0.002}$ \\
3.9 & 11 & 0.031 & 0.0025 & $0.018 \pm 0.003_{-0.001}^{+0.001}$ \\
3.9 & 11 & 0.031 & 0.0065 & $0.017 \pm 0.002_{-0.001}^{+0.001}$ \\
3.9 & 11 & 0.031 & 0.0150 & $0.017 \pm 0.002_{-0.002}^{+0.001}$ \\
3.9 & 11 & 0.031 & 0.0300 & $0.020 \pm 0.003_{-0.001}^{+0.001}$ \\
3.9 & 11 & 0.031 & 0.0500 & $0.017 \pm 0.003_{-0.003}^{+0.001}$ \\
3.9 & 11 & 0.031 & 0.0700 & $0.023 \pm 0.003_{-0.002}^{+0.002}$ \\
3.9 & 19 & 0.011 & 0.0065 & $0.031 \pm 0.005_{-0.005}^{+0.002}$ \\
3.9 & 19 & 0.011 & 0.0150 & $0.016 \pm 0.002_{-0.001}^{+0.002}$ \\
3.9 & 19 & 0.011 & 0.0300 & $0.019 \pm 0.003_{-0.003}^{+0.001}$ \\
3.9 & 19 & 0.011 & 0.0500 & $0.024 \pm 0.004_{-0.001}^{+0.004}$ \\
3.9 & 19 & 0.011 & 0.0700 & $0.028 \pm 0.003_{-0.002}^{+0.001}$ \\
3.9 & 19 & 0.011 & 0.0900 & $0.032 \pm 0.004_{-0.002}^{+0.001}$ \\
3.9 & 32 & 0.004 & 0.0300 & $0.027 \pm 0.004_{-0.004}^{+0.001}$ \\
3.9 & 32 & 0.004 & 0.0500 & $0.026 \pm 0.004_{-0.003}^{+0.003}$ \\
3.9 & 32 & 0.004 & 0.0700 & $0.030 \pm 0.004_{-0.003}^{+0.003}$ \\
3.9 & 32 & 0.004 & 0.0900 & $0.040 \pm 0.005_{-0.003}^{+0.002}$ \\
7.1 & 3 & 0.441 & 0.0003 & $0.045 \pm 0.004_{-0.003}^{+0.003}$ \\
7.1 & 3 & 0.441 & 0.0009 & $0.039 \pm 0.003_{-0.003}^{+0.002}$ \\
7.1 & 3 & 0.441 & 0.0025 & $0.032 \pm 0.003_{-0.002}^{+0.002}$ \\
7.1 & 3 & 0.441 & 0.0065 & $0.028 \pm 0.003_{-0.002}^{+0.001}$ \\
7.1 & 6 & 0.165 & 0.0009 & $0.034 \pm 0.003_{-0.002}^{+0.002}$ \\
7.1 & 6 & 0.165 & 0.0025 & $0.024 \pm 0.002_{-0.001}^{+0.002}$ \\
7.1 & 6 & 0.165 & 0.0065 & $0.019 \pm 0.002_{-0.001}^{+0.001}$ \\
7.1 & 6 & 0.165 & 0.0150 & $0.019 \pm 0.002_{-0.001}^{+0.002}$ \\
\hline
\end{tabular}




\begin{tabular}{|c|c|c|c|c|}
\hline$Q^{2}\left(\mathrm{GeV}^{2}\right)$ & $M_{X}(\mathrm{GeV})$ & $\beta$ & $x_{I P}$ & $\begin{array}{c}x_{I P} \sigma_{r}^{D(3)} \\
\pm \text { stat. } \pm \text { syst. }\end{array}$ \\
\hline 7.1 & 11 & 0.055 & 0.0025 & $0.024 \pm 0.003_{-0.002}^{+0.003}$ \\
7.1 & 11 & 0.055 & 0.0065 & $0.022 \pm 0.002_{-0.001}^{+0.002}$ \\
7.1 & 11 & 0.055 & 0.0150 & $0.018 \pm 0.002_{-0.002}^{+0.001}$ \\
7.1 & 11 & 0.055 & 0.0300 & $0.022 \pm 0.002_{-0.002}^{+0.002}$ \\
7.1 & 11 & 0.055 & 0.0500 & $0.026 \pm 0.003_{-0.002}^{+0.002}$ \\
7.1 & 11 & 0.055 & 0.0700 & $0.026 \pm 0.003_{-0.001}^{+0.002}$ \\
7.1 & 19 & 0.019 & 0.0065 & $0.034 \pm 0.005_{-0.006}^{+0.002}$ \\
7.1 & 19 & 0.019 & 0.0150 & $0.024 \pm 0.003_{-0.002}^{+0.003}$ \\
7.1 & 19 & 0.019 & 0.0300 & $0.024 \pm 0.003_{-0.001}^{+0.002}$ \\
7.1 & 19 & 0.019 & 0.0500 & $0.030 \pm 0.004_{-0.002}^{+0.003}$ \\
7.1 & 19 & 0.019 & 0.0700 & $0.028 \pm 0.003_{-0.002}^{+0.002}$ \\
7.1 & 19 & 0.019 & 0.0900 & $0.038 \pm 0.004_{-0.002}^{+0.004}$ \\
7.1 & 32 & 0.007 & 0.0300 & $0.029 \pm 0.003_{-0.002}^{+0.003}$ \\
7.1 & 32 & 0.007 & 0.0500 & $0.033 \pm 0.004_{-0.002}^{+0.004}$ \\
7.1 & 32 & 0.007 & 0.0700 & $0.032 \pm 0.003_{-0.002}^{+0.002}$ \\
7.1 & 32 & 0.007 & 0.0900 & $0.046 \pm 0.004_{-0.003}^{+0.004}$ \\
14 & 3 & 0.609 & 0.0009 & $0.036 \pm 0.004_{-0.002}^{+0.002}$ \\
14 & 3 & 0.609 & 0.0025 & $0.030 \pm 0.004_{-0.001}^{+0.002}$ \\
14 & 3 & 0.609 & 0.0065 & $0.035 \pm 0.004_{-0.001}^{+0.002}$ \\
14 & 6 & 0.280 & 0.0009 & $0.039 \pm 0.005_{-0.002}^{+0.002}$ \\
14 & 6 & 0.280 & 0.0025 & $0.031 \pm 0.003_{-0.001}^{+0.002}$ \\
14 & 6 & 0.280 & 0.0065 & $0.025 \pm 0.003_{-0.003}^{+0.002}$ \\
14 & 11 & 0.280 & 0.0150 & $0.026 \pm 0.003_{-0.001}^{+0.003}$ \\
14 & 11 & 0.280 & 0.0300 & $0.022 \pm 0.004_{-0.002}^{+0.004}$ \\
14 & 0.0 .104 & 0.104 & 0.0500 & $0.031 \pm 0.004_{-0.002}^{+0.002}$ \\
14 & 11 & 0.104 & 0.0700 & $0.032 \pm 0.004_{-0.003}^{+0.002}$ \\
\hline
\end{tabular}




\begin{tabular}{|c|c|c|c|c|}
\hline$Q^{2}\left(\mathrm{GeV}^{2}\right)$ & $M_{X}(\mathrm{GeV})$ & $\beta$ & $x_{\mathbb{P}}$ & $\begin{array}{c}x_{\mathbb{P}} \sigma_{r}^{D(3)} \\
\pm \text { stat. } \pm \text { syst. }\end{array}$ \\
\hline 14 & 19 & 0.037 & 0.0150 & $0.027 \pm 0.004_{-0.002}^{+0.001}$ \\
\hline 14 & 19 & 0.037 & 0.0300 & $0.028 \pm 0.003_{-0.002}^{+0.002}$ \\
\hline 14 & 19 & 0.037 & 0.0500 & $0.035 \pm 0.005_{-0.006}^{+0.003}$ \\
\hline 14 & 19 & 0.037 & 0.0700 & $0.036 \pm 0.004_{-0.002}^{+0.002}$ \\
\hline 14 & 19 & 0.037 & 0.0900 & $0.033 \pm 0.003_{-0.003}^{+0.002}$ \\
\hline 14 & 32 & 0.013 & 0.0300 & $0.036 \pm 0.005_{-0.006}^{+0.002}$ \\
\hline 14 & 32 & 0.013 & 0.0500 & $0.035 \pm 0.005_{-0.003}^{+0.002}$ \\
\hline 14 & 32 & 0.013 & 0.0700 & $0.038 \pm 0.004_{-0.003}^{+0.003}$ \\
\hline 14 & 32 & 0.013 & 0.0900 & $0.048 \pm 0.005_{-0.002}^{+0.003}$ \\
\hline 40 & 3 & 0.816 & 0.0009 & $0.030 \pm 0.006_{-0.001}^{+0.003}$ \\
\hline 40 & 3 & 0.816 & 0.0025 & $0.028 \pm 0.005_{-0.002}^{+0.004}$ \\
\hline 40 & 3 & 0.816 & 0.0065 & $0.024 \pm 0.004_{-0.002}^{+0.001}$ \\
\hline 40 & 3 & 0.816 & 0.0150 & $0.020 \pm 0.005_{-0.002}^{+0.001}$ \\
\hline 40 & 6 & 0.526 & 0.0025 & $0.034 \pm 0.004_{-0.003}^{+0.002}$ \\
\hline 40 & 6 & 0.526 & 0.0065 & $0.033 \pm 0.004_{-0.003}^{+0.002}$ \\
\hline 40 & 6 & 0.526 & 0.0150 & $0.029 \pm 0.004_{-0.002}^{+0.002}$ \\
\hline 40 & 6 & 0.526 & 0.0300 & $0.029 \pm 0.005_{-0.002}^{+0.004}$ \\
\hline 40 & 11 & 0.248 & 0.0065 & $0.021 \pm 0.003_{-0.001}^{+0.003}$ \\
\hline 40 & 11 & 0.248 & 0.0150 & $0.028 \pm 0.004_{-0.003}^{+0.002}$ \\
\hline 40 & 11 & 0.248 & 0.0300 & $0.023 \pm 0.003_{-0.001}^{+0.002}$ \\
\hline 40 & 11 & 0.248 & 0.0500 & $0.025 \pm 0.004_{-0.003}^{+0.002}$ \\
\hline 40 & 11 & 0.248 & 0.0700 & $0.033 \pm 0.004_{-0.003}^{+0.002}$ \\
\hline 40 & 11 & 0.248 & 0.0900 & $0.033 \pm 0.004_{-0.001}^{+0.003}$ \\
\hline 40 & 19 & 0.100 & 0.0150 & $0.030 \pm 0.004_{-0.004}^{+0.003}$ \\
\hline 40 & 19 & 0.100 & 0.0300 & $0.024 \pm 0.003_{-0.002}^{+0.001}$ \\
\hline 40 & 19 & 0.100 & 0.0500 & $0.039 \pm 0.005_{-0.004}^{+0.003}$ \\
\hline 40 & 19 & 0.100 & 0.0700 & $0.036 \pm 0.003_{-0.002}^{+0.002}$ \\
\hline 40 & 19 & 0.100 & 0.0900 & $0.043 \pm 0.004_{-0.003}^{+0.002}$ \\
\hline 40 & 32 & 0.038 & 0.0300 & $0.033 \pm 0.004_{-0.002}^{+0.003}$ \\
\hline 40 & 32 & 0.038 & 0.0500 & $0.044 \pm 0.005_{-0.005}^{+0.003}$ \\
\hline 40 & 32 & 0.038 & 0.0700 & $0.042 \pm 0.004_{-0.002}^{+0.003}$ \\
\hline 40 & 32 & 0.038 & 0.0900 & $0.056 \pm 0.005_{-0.002}^{+0.003}$ \\
\hline
\end{tabular}




\begin{tabular}{|c|c|c|c|c|}
\hline$Q^{2}\left(\mathrm{GeV}^{2}\right)$ & $M_{X}(\mathrm{GeV})$ & $\beta$ & $x_{\mathbb{P}}$ & $\begin{array}{c}x_{\mathbb{P}} \sigma_{r}^{D(3)} \\
\pm \text { stat. } \pm \text { syst. }\end{array}$ \\
\hline 2.5 & 3 & 0.217 & 0.0003 & $0.0448 \pm 0.0005_{-0.0049}^{+0.0053}$ \\
2.5 & 3 & 0.217 & 0.0005 & $0.0308 \pm 0.0003_{-0.0027}^{+0.0030}$ \\
2.5 & 3 & 0.217 & 0.0010 & $0.0243 \pm 0.0003_{-0.0017}^{+0.0018}$ \\
2.5 & 3 & 0.217 & 0.0020 & $0.0195 \pm 0.0003_{-0.0010}^{+0.0012}$ \\
2.5 & 3 & 0.217 & 0.0038 & $0.0182 \pm 0.0003_{-0.0006}^{+0.0007}$ \\
2.5 & 5 & 0.091 & 0.0005 & $0.0320 \pm 0.0006_{-0.0030}^{+0.0032}$ \\
2.5 & 5 & 0.091 & 0.0010 & $0.0219 \pm 0.0004_{-0.0015}^{+0.0017}$ \\
2.5 & 5 & 0.091 & 0.0020 & $0.0175 \pm 0.0003_{-0.0009}^{+0.0009}$ \\
2.5 & 5 & 0.091 & 0.0038 & $0.0158 \pm 0.0003_{-0.0006}^{+0.0007}$ \\
2.5 & 5 & 0.091 & 0.0073 & $0.0144 \pm 0.0003_{-0.0005}^{+0.0007}$ \\
2.5 & 5 & 0.091 & 0.0140 & $0.0159 \pm 0.0005_{-0.0009}^{+0.0016}$ \\
2.5 & 8 & 0.038 & 0.0020 & $0.0186 \pm 0.0003_{-0.0010}^{+0.0010}$ \\
2.5 & 8 & 0.038 & 0.0038 & $0.0161 \pm 0.0003_{-0.0005}^{+0.0006}$ \\
2.5 & 8 & 0.038 & 0.0073 & $0.0146 \pm 0.0003_{-0.0005}^{+0.0005}$ \\
2.5 & 13 & 0.015 & 0.0038 & $0.0226 \pm 0.0004_{-0.0007}^{+0.0008}$ \\
2.5 & 20 & 0.006 & 0.0140 & $0.0251 \pm 0.0031_{-0.0033}^{+0.0019}$ \\
3.5 & 3 & 0.280 & 0.0003 & $0.0429 \pm 0.0006_{-0.0045}^{+0.0052}$ \\
3.5 & 3 & 0.280 & 0.0005 & $0.0336 \pm 0.0004_{-0.0030}^{+0.0033}$ \\
3.5 & 3 & 0.280 & 0.0010 & $0.0273 \pm 0.0004_{-0.0019}^{+0.0020}$ \\
3.5 & 3 & 0.280 & 0.0020 & $0.0230 \pm 0.0003_{-0.0012}^{+0.0012}$ \\
3.5 & 3 & 0.280 & 0.0038 & $0.0207 \pm 0.0003_{-0.0007}^{+0.0008}$ \\
3.5 & 3 & 0.280 & 0.0073 & $0.0199 \pm 0.0005_{-0.0005}^{+0.0006}$ \\
3.5 & 5 & 0.123 & 0.0005 & $0.0303 \pm 0.0006_{-0.0029}^{+0.0032}$ \\
3.5 & 5 & 0.123 & 0.0010 & $0.0232 \pm 0.0004_{-0.0016}^{+0.0020}$ \\
3.5 & 5 & 0.123 & 0.0020 & $0.0195 \pm 0.0004_{-0.0010}^{+0.0011}$ \\
3.5 & 5 & 0.123 & 0.0038 & $0.0176 \pm 0.0004_{-0.0008}^{+0.0006}$ \\
3.5 & 5 & 0.123 & 0.0073 & $0.0171 \pm 0.0004_{-0.0005}^{+0.0004}$ \\
\hline
\end{tabular}

Table 9: The reduced diffractive cross section multiplied by $x_{\mathbb{P}}, x_{\mathbb{P}} \sigma_{r}^{D(3)}$, obtained with the LRG method for different values of $Q^{2}, M_{X}$ and $x_{\mathbb{I P}}$. The corresponding $\beta$ values are also indicated. The table continues on the next 8 pages. 


\begin{tabular}{|c|c|c|c|c|}
\hline$Q^{2}\left(\mathrm{GeV}^{2}\right)$ & $M_{X}(\mathrm{GeV})$ & $\beta$ & $x_{\mathbb{P}}$ & $\begin{array}{c}x_{\mathbb{P}} \sigma_{r}^{D(3)} \\
\pm \text { stat. } \pm \text { syst. }\end{array}$ \\
\hline 3.5 & 8 & 0.052 & 0.0020 & $0.0187 \pm 0.0003_{-0.0010}^{+0.0011}$ \\
\hline 3.5 & 8 & 0.052 & 0.0038 & $0.0165 \pm 0.0003_{-0.0005}^{+0.0006}$ \\
\hline 3.5 & 8 & 0.052 & 0.0073 & $0.0160 \pm 0.0003_{-0.0005}^{+0.0004}$ \\
\hline 3.5 & 13 & 0.020 & 0.0038 & $0.0205 \pm 0.0004_{-0.0007}^{+0.0009}$ \\
\hline 4.5 & 3 & 0.333 & 0.0003 & $0.0392 \pm 0.0006_{-0.0041}^{+0.0045}$ \\
\hline 4.5 & 3 & 0.333 & 0.0005 & $0.0340 \pm 0.0005_{-0.0029}^{+0.0035}$ \\
\hline 4.5 & 3 & 0.333 & 0.0010 & $0.0289 \pm 0.0005_{-0.0020}^{+0.0022}$ \\
\hline 4.5 & 3 & 0.333 & 0.0020 & $0.0250 \pm 0.0005_{-0.0013}^{+0.0014}$ \\
\hline 4.5 & 3 & 0.333 & 0.0038 & $0.0228 \pm 0.0004_{-0.0008}^{+0.0009}$ \\
\hline 4.5 & 3 & 0.333 & 0.0073 & $0.0217 \pm 0.0006_{-0.0006}^{+0.0006}$ \\
\hline 4.5 & 5 & 0.153 & 0.0005 & $0.0295 \pm 0.0007_{-0.0027}^{+0.0029}$ \\
\hline 4.5 & 5 & 0.153 & 0.0010 & $0.0232 \pm 0.0005_{-0.0017}^{+0.0020}$ \\
\hline 4.5 & 5 & 0.153 & 0.0020 & $0.0192 \pm 0.0005_{-0.0011}^{+0.0011}$ \\
\hline 4.5 & 5 & 0.153 & 0.0038 & $0.0183 \pm 0.0005_{-0.0007}^{+0.0008}$ \\
\hline 4.5 & 5 & 0.153 & 0.0073 & $0.0178 \pm 0.0005_{-0.0006}^{+0.0006}$ \\
\hline 4.5 & 5 & 0.153 & 0.0140 & $0.0203 \pm 0.0008_{-0.0009}^{+0.0011}$ \\
\hline 4.5 & 8 & 0.066 & 0.0020 & $0.0191 \pm 0.0004_{-0.0010}^{+0.0012}$ \\
\hline 4.5 & 8 & 0.066 & 0.0038 & $0.0171 \pm 0.0004_{-0.0007}^{+0.0009}$ \\
\hline 4.5 & 8 & 0.066 & 0.0073 & $0.0153 \pm 0.0004_{-0.0004}^{+0.0005}$ \\
\hline 4.5 & 13 & 0.026 & 0.0038 & $0.0197 \pm 0.0004_{-0.0008}^{+0.0006}$ \\
\hline 4.5 & 13 & 0.026 & 0.0073 & $0.0182 \pm 0.0004_{-0.0005}^{+0.0007}$ \\
\hline 5.5 & 3 & 0.379 & 0.0003 & $0.0405 \pm 0.0008_{-0.0042}^{+0.0045}$ \\
\hline 5.5 & 3 & 0.379 & 0.0005 & $0.0361 \pm 0.0006_{-0.0031}^{+0.0035}$ \\
\hline 5.5 & 3 & 0.379 & 0.0010 & $0.0311 \pm 0.0006_{-0.0021}^{+0.0024}$ \\
\hline 5.5 & 3 & 0.379 & 0.0020 & $0.0257 \pm 0.0005_{-0.0013}^{+0.0014}$ \\
\hline 5.5 & 3 & 0.379 & 0.0038 & $0.0232 \pm 0.0005_{-0.0009}^{+0.0013}$ \\
\hline 5.5 & 3 & 0.379 & 0.0073 & $0.0221 \pm 0.0006_{-0.0005}^{+0.0009}$ \\
\hline 5.5 & 5 & 0.180 & 0.0005 & $0.0306 \pm 0.0008_{-0.0027}^{+0.0030}$ \\
\hline 5.5 & 5 & 0.180 & 0.0010 & $0.0245 \pm 0.0005_{-0.0018}^{+0.0019}$ \\
\hline 5.5 & 5 & 0.180 & 0.0020 & $0.0192 \pm 0.0005_{-0.0009}^{+0.0012}$ \\
\hline 5.5 & 5 & 0.180 & 0.0038 & $0.0189 \pm 0.0005_{-0.0007}^{+0.0008}$ \\
\hline 5.5 & 5 & 0.180 & 0.0073 & $0.0169 \pm 0.0005_{-0.0004}^{+0.0009}$ \\
\hline 5.5 & 5 & 0.180 & 0.0140 & $0.0188 \pm 0.0008_{-0.0008}^{+0.0010}$ \\
\hline
\end{tabular}




\begin{tabular}{|c|c|c|c|c|}
\hline$Q^{2}\left(\mathrm{GeV}^{2}\right)$ & $M_{X}(\mathrm{GeV})$ & $\beta$ & $x_{\mathbb{P}}$ & $x_{\mathbb{P}} \sigma_{r}^{D(3)}$ \\
\hline 5.5 & 8 & 0.079 & 0.0020 & $0.0183 \pm 0.0004_{-0.0009}^{+0.0010}$ \\
5.5 & 8 & 0.079 & 0.0038 & $0.0172 \pm 0.0004_{-0.0006}^{+0.0007}$ \\
5.5 & 8 & 0.079 & 0.0073 & $0.0155 \pm 0.0004_{-0.0003}^{+0.0006}$ \\
5.5 & 8 & 0.079 & 0.0140 & $0.0182 \pm 0.0006_{-0.0008}^{+0.0010}$ \\
5.5 & 13 & 0.032 & 0.0038 & $0.0209 \pm 0.0005_{-0.0006}^{+0.0006}$ \\
5.5 & 13 & 0.032 & 0.0073 & $0.0199 \pm 0.0005_{-0.0005}^{+0.0005}$ \\
5.5 & 20 & 0.014 & 0.0073 & $0.0227 \pm 0.0007_{-0.0003}^{+0.0008}$ \\
6.5 & 3 & 0.419 & 0.0003 & $0.0417 \pm 0.0010_{-0.0043}^{+0.0047}$ \\
6.5 & 3 & 0.419 & 0.0005 & $0.0396 \pm 0.0007_{-0.0034}^{+0.0037}$ \\
6.5 & 3 & 0.419 & 0.0010 & $0.0319 \pm 0.0006_{-0.0022}^{+0.0024}$ \\
6.5 & 3 & 0.419 & 0.0020 & $0.0286 \pm 0.0006_{-0.0014}^{+0.0019}$ \\
6.5 & 3 & 0.419 & 0.0038 & $0.0255 \pm 0.0006_{-0.0008}^{+0.0012}$ \\
6.5 & 3 & 0.419 & 0.0073 & $0.0237 \pm 0.0006_{-0.0007}^{+0.0008}$ \\
6.5 & 5 & 0.206 & 0.0010 & $0.0265 \pm 0.0006_{-0.0020}^{+0.0020}$ \\
6.5 & 5 & 0.206 & 0.0020 & $0.0232 \pm 0.0006_{-0.0012}^{+0.0012}$ \\
6.5 & 5 & 0.206 & 0.0038 & $0.0194 \pm 0.0006_{-0.0008}^{+0.0008}$ \\
6.5 & 5 & 0.206 & 0.0073 & $0.0180 \pm 0.0006_{-0.0005}^{+0.0006}$ \\
6.5 & 8 & 0.092 & 0.0020 & $0.0195 \pm 0.0005_{-0.0010}^{+0.0010}$ \\
6.5 & 8 & 0.092 & 0.0038 & $0.0182 \pm 0.0005_{-0.0006}^{+0.0007}$ \\
6.5 & 8 & 0.092 & 0.0073 & $0.0176 \pm 0.0005_{-0.0005}^{+0.0007}$ \\
6.5 & 13 & 0.037 & 0.0038 & $0.0207 \pm 0.0006_{-0.0008}^{+0.0007}$ \\
6.5 & 13 & 0.037 & 0.0073 & $0.0181 \pm 0.0005_{-0.0005}^{+0.0007}$ \\
6.5 & 20 & 0.016 & 0.0073 & $0.0246 \pm 0.0009_{-0.0005}^{+0.0008}$ \\
8.5 & 3 & 0.486 & 0.0005 & $0.0409 \pm 0.0005_{-0.0035}^{+0.0038}$ \\
8.5 & 3 & 0.486 & 0.0010 & $0.0360 \pm 0.0005_{-0.0025}^{+0.0027}$ \\
8.5 & 3 & 0.486 & 0.0020 & $0.0305 \pm 0.0005_{-0.0016}^{+0.0017}$ \\
8.5 & 0.5 & 0.486 & 0.0073 & $0.0261 \pm 0.0005_{-0.0008}^{+0.0007}$ \\
8.5 & 5 & 0.254 & 0.0010 & $0.0319 \pm 0.0005_{-0.0022}^{+0.0023}$ \\
8.5 & 354 & 0.0020 & $0.0245 \pm 0.0004_{-0.0013}^{+0.0013}$ \\
\hline & 5 & 0.0038 & $0.0225 \pm 0.0004_{-0.0009}^{+0.0008}$ \\
\hline 8 & & 0.0073 & $0.0205 \pm 0.0004_{-0.0004}^{+0.0006}$ \\
\hline
\end{tabular}




\begin{tabular}{|c|c|c|c|c|}
\hline$Q^{2}\left(\mathrm{GeV}^{2}\right)$ & $M_{X}(\mathrm{GeV})$ & $\beta$ & $x_{\mathbb{P}}$ & $\begin{array}{c}\quad x_{I P} \sigma_{r}^{D(3)} \\
\pm \text { stat. } \pm \text { syst. }\end{array}$ \\
\hline 8.5 & 8 & 0.117 & 0.0020 & $0.0224 \pm 0.0004_{-0.0011}^{+0.0011}$ \\
\hline 8.5 & 8 & 0.117 & 0.0038 & $0.0195 \pm 0.0003_{-0.0006}^{+0.0007}$ \\
\hline 8.5 & 8 & 0.117 & 0.0073 & $0.0186 \pm 0.0003_{-0.0005}^{+0.0007}$ \\
\hline 8.5 & 13 & 0.048 & 0.0038 & $0.0236 \pm 0.0004_{-0.0007}^{+0.0008}$ \\
\hline 8.5 & 13 & 0.048 & 0.0073 & $0.0221 \pm 0.0004_{-0.0007}^{+0.0006}$ \\
\hline 8.5 & 20 & 0.021 & 0.0073 & $0.0273 \pm 0.0006_{-0.0005}^{+0.0009}$ \\
\hline 12 & 3 & 0.571 & 0.0005 & $0.0451 \pm 0.0007_{-0.0040}^{+0.0042}$ \\
\hline 12 & 3 & 0.571 & 0.0010 & $0.0396 \pm 0.0006_{-0.0028}^{+0.0029}$ \\
\hline 12 & 3 & 0.571 & 0.0020 & $0.0339 \pm 0.0006_{-0.0018}^{+0.0019}$ \\
\hline 12 & 3 & 0.571 & 0.0038 & $0.0310 \pm 0.0006_{-0.0013}^{+0.0011}$ \\
\hline 12 & 3 & 0.571 & 0.0073 & $0.0288 \pm 0.0006_{-0.0006}^{+0.0011}$ \\
\hline 12 & 5 & 0.324 & 0.0010 & $0.0348 \pm 0.0006_{-0.0024}^{+0.0025}$ \\
\hline 12 & 5 & 0.324 & 0.0020 & $0.0304 \pm 0.0006_{-0.0016}^{+0.0017}$ \\
\hline 12 & 5 & 0.324 & 0.0038 & $0.0249 \pm 0.0005_{-0.0008}^{+0.0009}$ \\
\hline 12 & 5 & 0.324 & 0.0073 & $0.0228 \pm 0.0005_{-0.0006}^{+0.0008}$ \\
\hline 12 & 8 & 0.158 & 0.0020 & $0.0253 \pm 0.0005_{-0.0015}^{+0.0013}$ \\
\hline 12 & 8 & 0.158 & 0.0038 & $0.0216 \pm 0.0004_{-0.0007}^{+0.0008}$ \\
\hline 12 & 8 & 0.158 & 0.0073 & $0.0204 \pm 0.0004_{-0.0005}^{+0.0005}$ \\
\hline 12 & 8 & 0.158 & 0.0140 & $0.0219 \pm 0.0005_{-0.0006}^{+0.0013}$ \\
\hline 12 & 13 & 0.066 & 0.0038 & $0.0255 \pm 0.0006_{-0.0008}^{+0.0007}$ \\
\hline 12 & 13 & 0.066 & 0.0073 & $0.0230 \pm 0.0004_{-0.0004}^{+0.0005}$ \\
\hline 12 & 13 & 0.066 & 0.0140 & $0.0260 \pm 0.0006_{-0.0009}^{+0.0021}$ \\
\hline 12 & 20 & 0.029 & 0.0073 & $0.0285 \pm 0.0008_{-0.0005}^{+0.0011}$ \\
\hline 12 & 20 & 0.029 & 0.0140 & $0.0285 \pm 0.0006_{-0.0018}^{+0.0031}$ \\
\hline 16 & 3 & 0.640 & 0.0005 & $0.0454 \pm 0.0012_{-0.0041}^{+0.0042}$ \\
\hline 16 & 3 & 0.640 & 0.0010 & $0.0412 \pm 0.0009_{-0.0028}^{+0.0031}$ \\
\hline 16 & 3 & 0.640 & 0.0020 & $0.0363 \pm 0.0008_{-0.0020}^{+0.0021}$ \\
\hline 16 & 3 & 0.640 & 0.0038 & $0.0306 \pm 0.0007_{-0.0011}^{+0.0011}$ \\
\hline 16 & 3 & 0.640 & 0.0073 & $0.0306 \pm 0.0008_{-0.0007}^{+0.0010}$ \\
\hline 16 & 3 & 0.640 & 0.0140 & $0.0307 \pm 0.0011_{-0.0017}^{+0.0018}$ \\
\hline
\end{tabular}




\begin{tabular}{|c|c|c|c|c|}
\hline$Q^{2}\left(\mathrm{GeV}^{2}\right)$ & $M_{X}(\mathrm{GeV})$ & $\beta$ & $x_{\mathbb{P}}$ & $x_{\mathbb{P}} \sigma_{r}^{D(3)}$ \\
\hline 16 & 5 & 0.390 & 0.0010 & $0.0398 \pm 0.0010_{-0.0029}^{+0.0030}$ \\
16 & 5 & 0.390 & 0.0020 & $0.0315 \pm 0.0008_{-0.0015}^{+0.0017}$ \\
16 & 5 & 0.390 & 0.0038 & $0.0284 \pm 0.0007_{-0.0010}^{+0.0010}$ \\
16 & 5 & 0.390 & 0.0073 & $0.0267 \pm 0.0007_{-0.0004}^{+0.0008}$ \\
16 & 5 & 0.390 & 0.0140 & $0.0275 \pm 0.0008_{-0.0017}^{+0.0017}$ \\
16 & 8 & 0.200 & 0.0020 & $0.0282 \pm 0.0007_{-0.0014}^{+0.0015}$ \\
16 & 8 & 0.200 & 0.0038 & $0.0240 \pm 0.0005_{-0.0008}^{+0.0008}$ \\
16 & 8 & 0.200 & 0.0073 & $0.0226 \pm 0.0005_{-0.0006}^{+0.0006}$ \\
16 & 8 & 0.200 & 0.0140 & $0.0244 \pm 0.0006_{-0.0007}^{+0.0010}$ \\
16 & 13 & 0.086 & 0.0038 & $0.0287 \pm 0.0008_{-0.0008}^{+0.0010}$ \\
16 & 13 & 0.086 & 0.0073 & $0.0245 \pm 0.0006_{-0.0006}^{+0.0005}$ \\
16 & 13 & 0.086 & 0.0140 & $0.0267 \pm 0.0007_{-0.0014}^{+0.0018}$ \\
16 & 20 & 0.038 & 0.0073 & $0.0315 \pm 0.0012_{-0.0011}^{+0.0007}$ \\
16 & 20 & 0.038 & 0.0140 & $0.0305 \pm 0.0008_{-0.0020}^{+0.0033}$ \\
22 & 3 & 0.710 & 0.0005 & $0.0416 \pm 0.0019_{-0.0033}^{+0.0044}$ \\
22 & 3 & 0.710 & 0.0010 & $0.0398 \pm 0.0011_{-0.0033}^{+0.0030}$ \\
22 & 3 & 0.710 & 0.0020 & $0.0350 \pm 0.0010_{-0.0021}^{+0.0022}$ \\
22 & 3 & 0.710 & 0.0038 & $0.0312 \pm 0.0009_{-0.0015}^{+0.0013}$ \\
22 & 3 & 0.710 & 0.0073 & $0.0293 \pm 0.0009_{-0.0011}^{+0.0013}$ \\
22 & 3 & 0.710 & 0.0140 & $0.0286 \pm 0.0010_{-0.0014}^{+0.0017}$ \\
22 & 5 & 0.468 & 0.0010 & $0.0442 \pm 0.0014_{-0.0030}^{+0.0030}$ \\
22 & 5 & 0.468 & 0.0020 & $0.0369 \pm 0.0011_{-0.0018}^{+0.0020}$ \\
22 & 5 & 0.468 & 0.0038 & $0.0319 \pm 0.0009_{-0.0013}^{+0.0012}$ \\
22 & 5 & 0.468 & 0.0073 & $0.0302 \pm 0.0009_{-0.0009}^{+0.0012}$ \\
22 & 5 & 0.468 & 0.0140 & $0.0283 \pm 0.0009_{-0.0011}^{+0.0027}$ \\
22 & 8 & 0.256 & 0.0020 & $0.0293 \pm 0.0008_{-0.0015}^{+0.0015}$ \\
22 & 8 & 0.256 & 0.0038 & $0.0260 \pm 0.0007_{-0.0008}^{+0.0011}$ \\
22 & 0.256 & 0.0073 & $0.0238 \pm 0.0006_{-0.0008}^{+0.0008}$ \\
\hline
\end{tabular}




\begin{tabular}{|c|c|c|c|c|}
\hline$Q^{2}\left(\mathrm{GeV}^{2}\right)$ & $M_{X}(\mathrm{GeV})$ & $\beta$ & $x_{\mathbb{P}}$ & $\begin{array}{c}x_{\mathbb{P}} \sigma_{r}^{D(3)} \\
\pm \text { stat. } \pm \text { syst. }\end{array}$ \\
\hline 22 & 20 & 0.052 & 0.0140 & $0.0310 \pm 0.0010_{-0.0013}^{+0.0026}$ \\
\hline 30 & 3 & 0.769 & 0.0010 & $0.0352 \pm 0.0013_{-0.0027}^{+0.0030}$ \\
\hline 30 & 3 & 0.769 & 0.0020 & $0.0303 \pm 0.0012_{-0.0022}^{+0.0017}$ \\
\hline 30 & 3 & 0.769 & 0.0038 & $0.0276 \pm 0.0011_{-0.0015}^{+0.0013}$ \\
\hline 30 & 3 & 0.769 & 0.0073 & $0.0286 \pm 0.0012_{-0.0014}^{+0.0007}$ \\
\hline 30 & 3 & 0.769 & 0.0140 & $0.0279 \pm 0.0013_{-0.0016}^{+0.0012}$ \\
\hline 30 & 5 & 0.545 & 0.0010 & $0.0430 \pm 0.0019_{-0.0027}^{+0.0041}$ \\
\hline 30 & 5 & 0.545 & 0.0020 & $0.0361 \pm 0.0013_{-0.0023}^{+0.0018}$ \\
\hline 30 & 5 & 0.545 & 0.0038 & $0.0330 \pm 0.0013_{-0.0012}^{+0.0018}$ \\
\hline 30 & 5 & 0.545 & 0.0073 & $0.0308 \pm 0.0012_{-0.0010}^{+0.0008}$ \\
\hline 30 & 5 & 0.545 & 0.0140 & $0.0302 \pm 0.0013_{-0.0010}^{+0.0028}$ \\
\hline 30 & 8 & 0.319 & 0.0020 & $0.0317 \pm 0.0011_{-0.0015}^{+0.0016}$ \\
\hline 30 & 8 & 0.319 & 0.0038 & $0.0271 \pm 0.0008_{-0.0009}^{+0.0009}$ \\
\hline 30 & 8 & 0.319 & 0.0073 & $0.0236 \pm 0.0008_{-0.0004}^{+0.0005}$ \\
\hline 30 & 8 & 0.319 & 0.0140 & $0.0270 \pm 0.0010_{-0.0010}^{+0.0007}$ \\
\hline 30 & 13 & 0.151 & 0.0038 & $0.0283 \pm 0.0011_{-0.0009}^{+0.0009}$ \\
\hline 30 & 13 & 0.151 & 0.0073 & $0.0258 \pm 0.0008_{-0.0009}^{+0.0007}$ \\
\hline 30 & 13 & 0.151 & 0.0140 & $0.0280 \pm 0.0010_{-0.0011}^{+0.0018}$ \\
\hline 30 & 20 & 0.070 & 0.0140 & $0.0294 \pm 0.0010_{-0.0011}^{+0.0033}$ \\
\hline 40 & 3 & 0.816 & 0.0010 & $0.0349 \pm 0.0021_{-0.0025}^{+0.0028}$ \\
\hline 40 & 3 & 0.816 & 0.0020 & $0.0293 \pm 0.0016_{-0.0018}^{+0.0018}$ \\
\hline 40 & 3 & 0.816 & 0.0038 & $0.0258 \pm 0.0015_{-0.0016}^{+0.0012}$ \\
\hline 40 & 3 & 0.816 & 0.0073 & $0.0227 \pm 0.0014_{-0.0013}^{+0.0011}$ \\
\hline 40 & 3 & 0.816 & 0.0140 & $0.0206 \pm 0.0014_{-0.0013}^{+0.0013}$ \\
\hline 40 & 5 & 0.615 & 0.0020 & $0.0357 \pm 0.0017_{-0.0019}^{+0.0026}$ \\
\hline 40 & 5 & 0.615 & 0.0038 & $0.0309 \pm 0.0016_{-0.0016}^{+0.0011}$ \\
\hline 40 & 5 & 0.615 & 0.0073 & $0.0282 \pm 0.0015_{-0.0012}^{+0.0005}$ \\
\hline 40 & 5 & 0.615 & 0.0140 & $0.0273 \pm 0.0015_{-0.0013}^{+0.0024}$ \\
\hline 40 & 8 & 0.385 & 0.0020 & $0.0386 \pm 0.0016_{-0.0027}^{+0.0021}$ \\
\hline 40 & 8 & 0.385 & 0.0038 & $0.0298 \pm 0.0011_{-0.0010}^{+0.0009}$ \\
\hline 40 & 8 & 0.385 & 0.0073 & $0.0252 \pm 0.0010_{-0.0007}^{+0.0008}$ \\
\hline 40 & 8 & 0.385 & 0.0140 & $0.0281 \pm 0.0012_{-0.0017}^{+0.0017}$ \\
\hline
\end{tabular}




\begin{tabular}{|c|c|c|c|c|}
\hline$Q^{2}\left(\mathrm{GeV}^{2}\right)$ & $M_{X}(\mathrm{GeV})$ & $\beta$ & $x_{\mathbb{P}}$ & $\begin{array}{c}x_{\mathbb{P}} \sigma_{r}^{D(3)} \\
\pm \text { stat. } \pm \text { syst. }\end{array}$ \\
\hline 40 & 13 & 0.191 & 0.0038 & $0.0317 \pm 0.0015_{-0.0009}^{+0.0010}$ \\
\hline 40 & 13 & 0.191 & 0.0073 & $0.0273 \pm 0.0010_{-0.0007}^{+0.0007}$ \\
\hline 40 & 13 & 0.191 & 0.0140 & $0.0271 \pm 0.0012_{-0.0010}^{+0.0020}$ \\
\hline 40 & 20 & 0.091 & 0.0140 & $0.0345 \pm 0.0014_{-0.0019}^{+0.0024}$ \\
\hline 50 & 3 & 0.847 & 0.0020 & $0.0304 \pm 0.0021_{-0.0025}^{+0.0022}$ \\
\hline 50 & 3 & 0.847 & 0.0038 & $0.0236 \pm 0.0017_{-0.0014}^{+0.0015}$ \\
\hline 50 & 3 & 0.847 & 0.0073 & $0.0209 \pm 0.0017_{-0.0011}^{+0.000}$ \\
\hline 50 & 3 & 0.847 & 0.0140 & $0.0221 \pm 0.0018_{-0.011}^{+0.0015}$ \\
\hline 50 & 5 & 0.667 & 0.0020 & $0.0360 \pm 0.0021_{-0.0017}^{+0.0024}$ \\
\hline 50 & 5 & 0.667 & 0.0038 & $0.0365 \pm 0.0021_{-0.0021}^{+0.0013}$ \\
\hline 50 & 5 & 0.667 & 0.0073 & $0.0256 \pm 0.0017_{-0.0012}^{+0.0015}$ \\
\hline 50 & 5 & 0.667 & 0.0140 & $0.0300 \pm 0.0020_{-0.0022}^{+0.0007}$ \\
\hline 50 & 8 & 0.439 & 0.0020 & $0.0396 \pm 0.0023_{-0.0024}^{+0.0018}$ \\
\hline 50 & 8 & 0.439 & 0.0038 & $0.0334 \pm 0.0014_{-0.0013}^{+0.0019}$ \\
\hline 50 & 8 & 0.439 & 0.0073 & $0.0290 \pm 0.0013_{-0.0007}^{+0.0011}$ \\
\hline 50 & 8 & 0.439 & 0.0140 & $0.0277 \pm 0.0014_{-0.0012}^{+0.0023}$ \\
\hline 50 & 13 & 0.228 & 0.0038 & $0.0352 \pm 0.0020_{-0.0016}^{+0.0010}$ \\
\hline 50 & 13 & 0.228 & 0.0073 & $0.0312 \pm 0.0013_{-0.0011}^{+0.0011}$ \\
\hline 50 & 13 & 0.228 & 0.0140 & $0.0284 \pm 0.0013_{-0.0012}^{+0.0011}$ \\
\hline 50 & 20 & 0.111 & 0.0140 & $0.0344 \pm 0.0015_{-0.0022}^{+0.0041}$ \\
\hline 65 & 3 & 0.878 & 0.0020 & $0.0282 \pm 0.0025_{-0.0026}^{+0.0016}$ \\
\hline 65 & 3 & 0.878 & 0.0038 & $0.0221 \pm 0.0020_{-0.012}^{+0.0015}$ \\
\hline 65 & 3 & 0.878 & 0.0073 & $0.0182 \pm 0.0018_{-0.0014}^{+0.0007}$ \\
\hline 65 & 3 & 0.878 & 0.0140 & $0.0148 \pm 0.0017_{-0.0011}^{+0.0013}$ \\
\hline 65 & 5 & 0.722 & 0.0020 & $0.0342 \pm 0.0028_{-0.0023}^{+0.0027}$ \\
\hline 65 & 5 & 0.722 & 0.0038 & $0.0307 \pm 0.0023_{-0.0012}^{+0.0014}$ \\
\hline 65 & 5 & 0.722 & 0.0073 & $0.0295 \pm 0.0022_{-0.0012}^{+0.0011}$ \\
\hline 65 & 5 & 0.722 & 0.0140 & $0.0258 \pm 0.0021_{-0.0005}^{+0.000}$ \\
\hline 65 & 8 & 0.504 & 0.0038 & $0.0324 \pm 0.0017_{-0.0010}^{+0.0011}$ \\
\hline 65 & 8 & 0.504 & 0.0073 & $0.0290 \pm 0.0015_{-0.0010}^{+0.0015}$ \\
\hline 65 & 8 & 0.504 & 0.0140 & $0.0274 \pm 0.0015_{-0.0005}^{+0.0013}$ \\
\hline
\end{tabular}




\begin{tabular}{|c|c|c|c|c|}
\hline$Q^{2}\left(\mathrm{GeV}^{2}\right)$ & $M_{X}(\mathrm{GeV})$ & $\beta$ & $x_{\mathbb{P}}$ & $\begin{array}{c}x_{\mathbb{P}} \sigma_{r}^{D(3)} \\
\pm \text { stat. } \pm \text { syst. }\end{array}$ \\
\hline 65 & 13 & 0.278 & 0.0073 & $0.0304 \pm 0.0014_{-0.0013}^{+0.0014}$ \\
\hline 65 & 13 & 0.278 & 0.0140 & $0.0297 \pm 0.0015_{-0.0015}^{+0.0014}$ \\
\hline 65 & 20 & 0.140 & 0.0140 & $0.0339 \pm 0.0017_{-0.0020}^{+0.0020}$ \\
\hline 85 & 3 & 0.904 & 0.0020 & $0.0188 \pm 0.0029_{-0.0013}^{+0.0028}$ \\
\hline 85 & 3 & 0.904 & 0.0038 & $0.0158 \pm 0.0021_{-0.0012}^{+0.0026}$ \\
\hline 85 & 3 & 0.904 & 0.0073 & $0.0165 \pm 0.0022_{-0.0010}^{+0.0015}$ \\
\hline 85 & 3 & 0.904 & 0.0140 & $0.0132 \pm 0.0020_{-0.0005}^{+0.0009}$ \\
\hline 85 & 5 & 0.773 & 0.0020 & $0.0329 \pm 0.0046_{-0.0014}^{+0.0027}$ \\
\hline 85 & 5 & 0.773 & 0.0038 & $0.0243 \pm 0.0024_{-0.0020}^{+0.0009}$ \\
\hline 85 & 5 & 0.773 & 0.0073 & $0.0225 \pm 0.0024_{-0.0005}^{+0.0016}$ \\
\hline 85 & 5 & 0.773 & 0.0140 & $0.0224 \pm 0.0023_{-0.0005}^{+0.0008}$ \\
\hline 85 & 8 & 0.570 & 0.0038 & $0.0321 \pm 0.0021_{-0.0011}^{+0.0016}$ \\
\hline 85 & 8 & 0.570 & 0.0073 & $0.0298 \pm 0.0019_{-0.0017}^{+0.0011}$ \\
\hline 85 & 8 & 0.570 & 0.0140 & $0.0260 \pm 0.0018_{-0.0015}^{+0.0020}$ \\
\hline 85 & 13 & 0.335 & 0.0073 & $0.0304 \pm 0.0017_{-0.0013}^{+0.0011}$ \\
\hline 85 & 13 & 0.335 & 0.0140 & $0.0301 \pm 0.0018_{-0.0013}^{+0.0013}$ \\
\hline 85 & 20 & 0.175 & 0.0140 & $0.0299 \pm 0.0018_{-0.0017}^{+0.0030}$ \\
\hline 110 & 3 & 0.924 & 0.0038 & $0.0180 \pm 0.0028_{-0.0027}^{+0.0009}$ \\
\hline 110 & 3 & 0.924 & 0.0073 & $0.0138 \pm 0.0022_{-0.0006}^{+0.0006}$ \\
\hline 110 & 3 & 0.924 & 0.0140 & $0.0106 \pm 0.0019_{-0.0007}^{+0.0011}$ \\
\hline 110 & 5 & 0.815 & 0.0038 & $0.0237 \pm 0.0028_{-0.0016}^{+0.0022}$ \\
\hline 110 & 5 & 0.815 & 0.0073 & $0.0205 \pm 0.0025_{-0.0015}^{+0.0013}$ \\
\hline 110 & 5 & 0.815 & 0.0140 & $0.0207 \pm 0.0024_{-0.0005}^{+0.0010}$ \\
\hline 110 & 8 & 0.632 & 0.0038 & $0.0334 \pm 0.0025_{-0.0014}^{+0.0010}$ \\
\hline 110 & 8 & 0.632 & 0.0073 & $0.0287 \pm 0.0021_{-0.0005}^{+0.0022}$ \\
\hline 110 & 8 & 0.632 & 0.0140 & $0.0265 \pm 0.0019_{-0.0020}^{+0.0017}$ \\
\hline 110 & 13 & 0.394 & 0.0073 & $0.0303 \pm 0.0019_{-0.0012}^{+0.0010}$ \\
\hline 110 & 13 & 0.394 & 0.0140 & $0.0263 \pm 0.0017_{-0.0007}^{+0.0017}$ \\
\hline 110 & 20 & 0.216 & 0.0140 & $0.0284 \pm 0.0018_{-0.0009}^{+0.0009}$ \\
\hline 140 & 3 & 0.940 & 0.0038 & $0.0146 \pm 0.0032_{-0.0024}^{+0.0014}$ \\
\hline 140 & 3 & 0.940 & 0.0073 & $0.0112 \pm 0.0026_{-0.0010}^{+0.0010}$ \\
\hline 140 & 3 & 0.940 & 0.0140 & $0.0091 \pm 0.0025_{-0.0006}^{+0.0017}$ \\
\hline
\end{tabular}




\begin{tabular}{|c|c|c|c|c|}
\hline$Q^{2}\left(\mathrm{GeV}^{2}\right)$ & $M_{X}(\mathrm{GeV})$ & $\beta$ & $x_{I P}$ & $x_{I P} \sigma_{r}^{D(3)}$ \\
\hline 140 & 5 & 0.848 & 0.0038 & $0.0188 \pm 0.0034_{-0.0021}^{+0.0022}$ \\
140 & 5 & 0.848 & 0.0073 & $0.0148 \pm 0.0027_{-0.0023}^{+0.0005}$ \\
140 & 5 & 0.848 & 0.0140 & $0.0155 \pm 0.0026_{-0.0007}^{+0.0013}$ \\
140 & 8 & 0.686 & 0.0038 & $0.0349 \pm 0.0042_{-0.0027}^{+0.0030}$ \\
140 & 8 & 0.686 & 0.0073 & $0.0280 \pm 0.0026_{-0.0022}^{+0.0004}$ \\
140 & 8 & 0.686 & 0.0140 & $0.0238 \pm 0.0023_{-0.0011}^{+0.0016}$ \\
140 & 13 & 0.453 & 0.0073 & $0.0302 \pm 0.0024_{-0.0012}^{+0.0011}$ \\
140 & 13 & 0.453 & 0.0140 & $0.0271 \pm 0.0021_{-0.0013}^{+0.0013}$ \\
140 & 20 & 0.259 & 0.0140 & $0.0288 \pm 0.0022_{-0.0004}^{+0.0043}$ \\
185 & 3 & 0.954 & 0.0038 & $0.0137 \pm 0.0057_{-0.0020}^{+0.0066}$ \\
185 & 3 & 0.954 & 0.0073 & $0.0101 \pm 0.0042_{-0.0001}^{+0.0014}$ \\
185 & 3 & 0.954 & 0.0140 & $0.0136 \pm 0.0045_{-0.0016}^{+0.0006}$ \\
185 & 5 & 0.881 & 0.0038 & $0.0110 \pm 0.0044_{-0.0053}^{+0.0003}$ \\
185 & 5 & 0.881 & 0.0073 & $0.0107 \pm 0.0029_{-0.0008}^{+0.0010}$ \\
185 & 5 & 0.881 & 0.0140 & $0.0113 \pm 0.0030_{-0.0014}^{+0.0012}$ \\
185 & 8 & 0.743 & 0.0073 & $0.0207 \pm 0.0029_{-0.0020}^{+0.0013}$ \\
185 & 8 & 0.743 & 0.0140 & $0.0186 \pm 0.0026_{-0.0006}^{+0.0015}$ \\
185 & 13 & 0.523 & 0.0073 & $0.0314 \pm 0.0034_{-0.0017}^{+0.0017}$ \\
185 & 13 & 0.523 & 0.0140 & $0.0234 \pm 0.0024_{-0.0017}^{+0.0016}$ \\
185 & 20 & 0.316 & 0.0140 & $0.0322 \pm 0.0031_{-0.0039}^{+0.0028}$ \\
255 & 3 & 0.966 & 0.0073 & $0.0061 \pm 0.0033_{-0.0000}^{+0.0003}$ \\
255 & 3 & 0.966 & 0.0140 & $0.0038 \pm 0.0023_{-0.0000}^{+0.0013}$ \\
255 & 5 & 0.911 & 0.0073 & $0.0095 \pm 0.0034_{-0.0001}^{+0.0027}$ \\
255 & 5 & 0.911 & 0.0140 & $0.0082 \pm 0.0029_{-0.0019}^{+0.0018}$ \\
255 & 8 & 0.799 & 0.0073 & $0.0170 \pm 0.0034_{-0.0026}^{+0.0023}$ \\
255 & 8 & 0.799 & 0.0140 & $0.0233 \pm 0.0036_{-0.0014}^{+0.0021}$ \\
255 & 13 & 0.601 & 0.0140 & $0.0238 \pm 0.0028_{-0.0014}^{+0.0011}$ \\
255 & 0.389 & 0.0140 & $0.0252 \pm 0.0031_{-0.0015}^{+0.0039}$ \\
\hline
\end{tabular}




\begin{tabular}{|c|c|c|}
\hline & Fit LPS (no interference) & Fit LPS max interf. $\mathbb{P}$ - $\mathbb{R}$ \\
\hline$\chi^{2} / \mathrm{ndf}$ & $162.9 / 153$ & $160.7 / 153$ \\
\hline$\alpha_{\mathbb{P}}(0)$ & $1.11 \pm 0.02$ (stat.) $)_{-0.02}^{+0.01}$ (syst.) \pm 0.02 (model) & $1.16 \pm 0.03$ (stat.) \\
\hline$\alpha_{\mathbb{R}}(0)$ & $0.75 \pm 0.07$ (stat.) $)_{-0.04}^{+0.02}$ (syst.) \pm 0.05 (model) & $0.68 \pm 0.09$ (stat.) \\
\hline$\alpha_{\mathbb{P}}^{\prime}$ & $-0.01 \pm 0.06$ (stat.) $)_{-0.08}^{+0.04}$ (syst.) $\pm 0.04\left(\right.$ model) $\mathrm{GeV}^{-2}$ & $0.12 \pm 0.05$ (stat.) $\mathrm{GeV}^{-2}$ \\
\hline$B_{\mathbb{P}}$ & $7.1 \pm 0.7$ (stat.) $)_{-0.7}^{+1.4}$ (syst.) $\mathrm{GeV}^{-2}$ & $5.2 \pm 0.5$ (stat.) $\mathrm{GeV}^{-2}$ \\
\hline$n_{\mathbb{R}}$ & $2.11 \pm 0.64$ (stat.) ${ }_{-0.37}^{+0.26}$ (syst.) & $1.9 \pm 0.7$ (stat.) \\
\hline
\end{tabular}

Table 10: The values and uncertainties of the parameters extracted from the Regge fits to the LPS data, without interference and with maximal interference between the Pomeron and Reggeon exchanges.

\begin{tabular}{|c|c|}
\hline & Fit LRG \\
\hline$\chi^{2} / \mathrm{ndf}$ & $\chi^{2} / \mathrm{ndf}=143.9 / 168$ \\
\hline$\alpha_{\mathbb{P}}(0)$ & $1.117 \pm 0.006_{-0.007}^{+0.022}($ model $)$ \\
\hline$\alpha_{\mathbb{R}}(0)$ & fixed to 0.75 \\
\hline$\alpha_{\mathbb{P}}^{\prime}$ & fixed to 0 \\
\hline$B_{\mathbb{P}}$ & fixed to $7.0 \mathrm{GeV}^{-2}$ \\
\hline$n_{\mathbb{R}}$ & $2.98 \pm 0.39$ \\
\hline
\end{tabular}

Table 11: The values of the parameters extracted from the Regge fit to the LRG data and the corresponding uncertainties. 


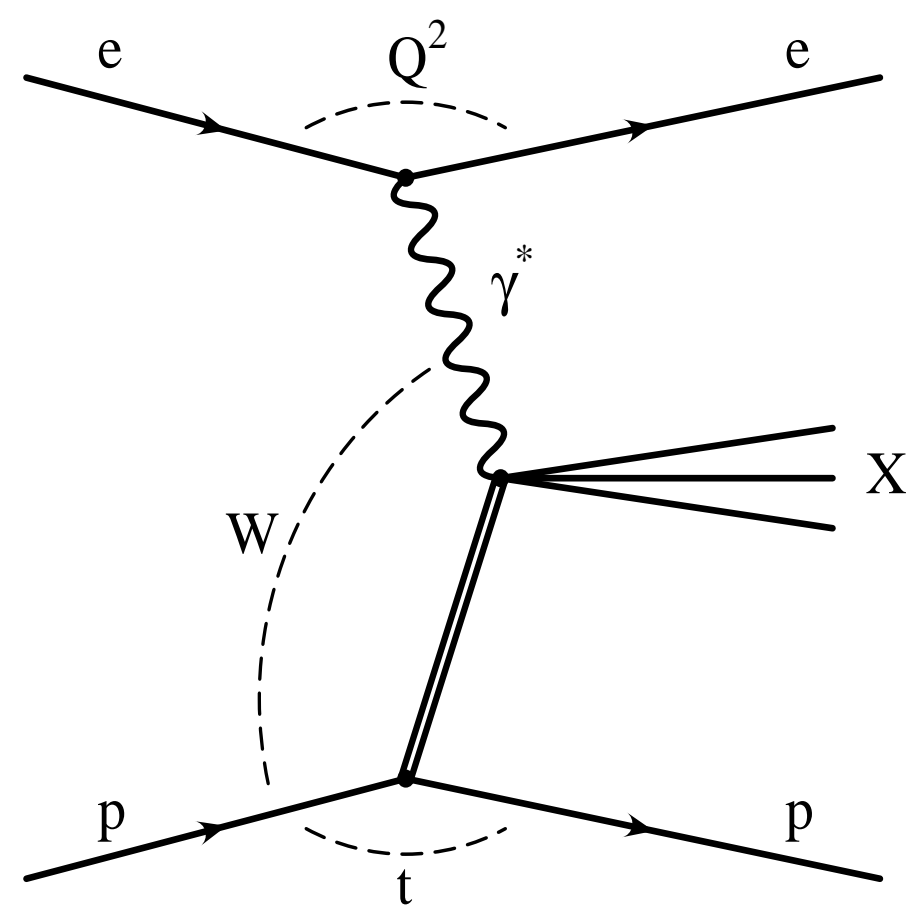

Figure 1: Schematic diagram of the reaction $e p \rightarrow e X p$. 


\section{ZEUS}
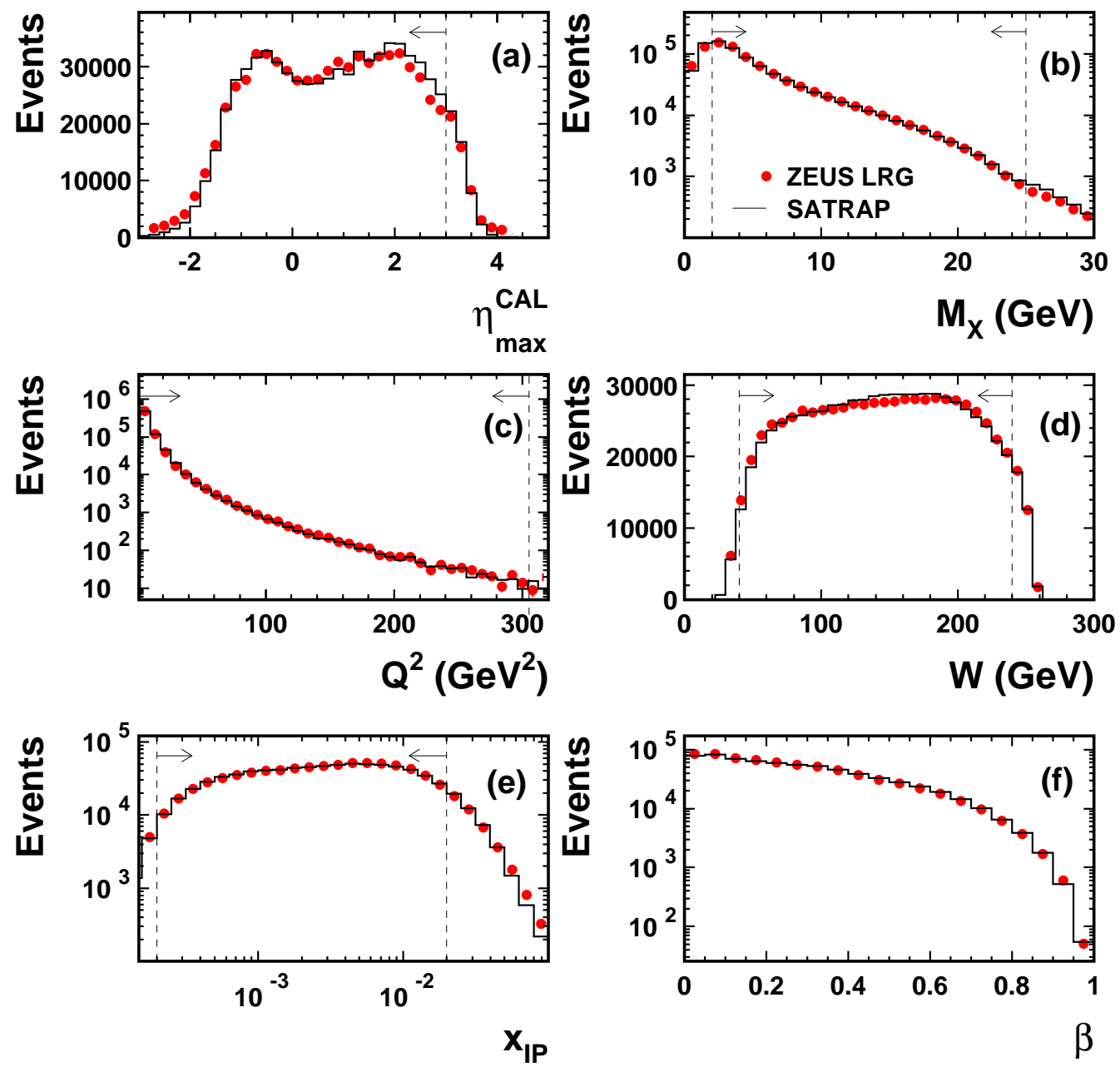

Figure 2: Comparison of the distributions measured (dots) and obtained with the reweighted SATRAP $M C$ (histograms) for (a) $\eta_{\max }^{\mathrm{CAL}}$, (b) $M_{X}$, (c) $Q^{2}$, (d) $W$, (e) $x_{\mathbb{I P}}$ and $(f) \beta$ in the $L R G$ analysis. For each plot, all analysis cuts have been applied except that on the plotted variable. The vertical lines with arrows indicate the selected region. 


\section{ZEUS}
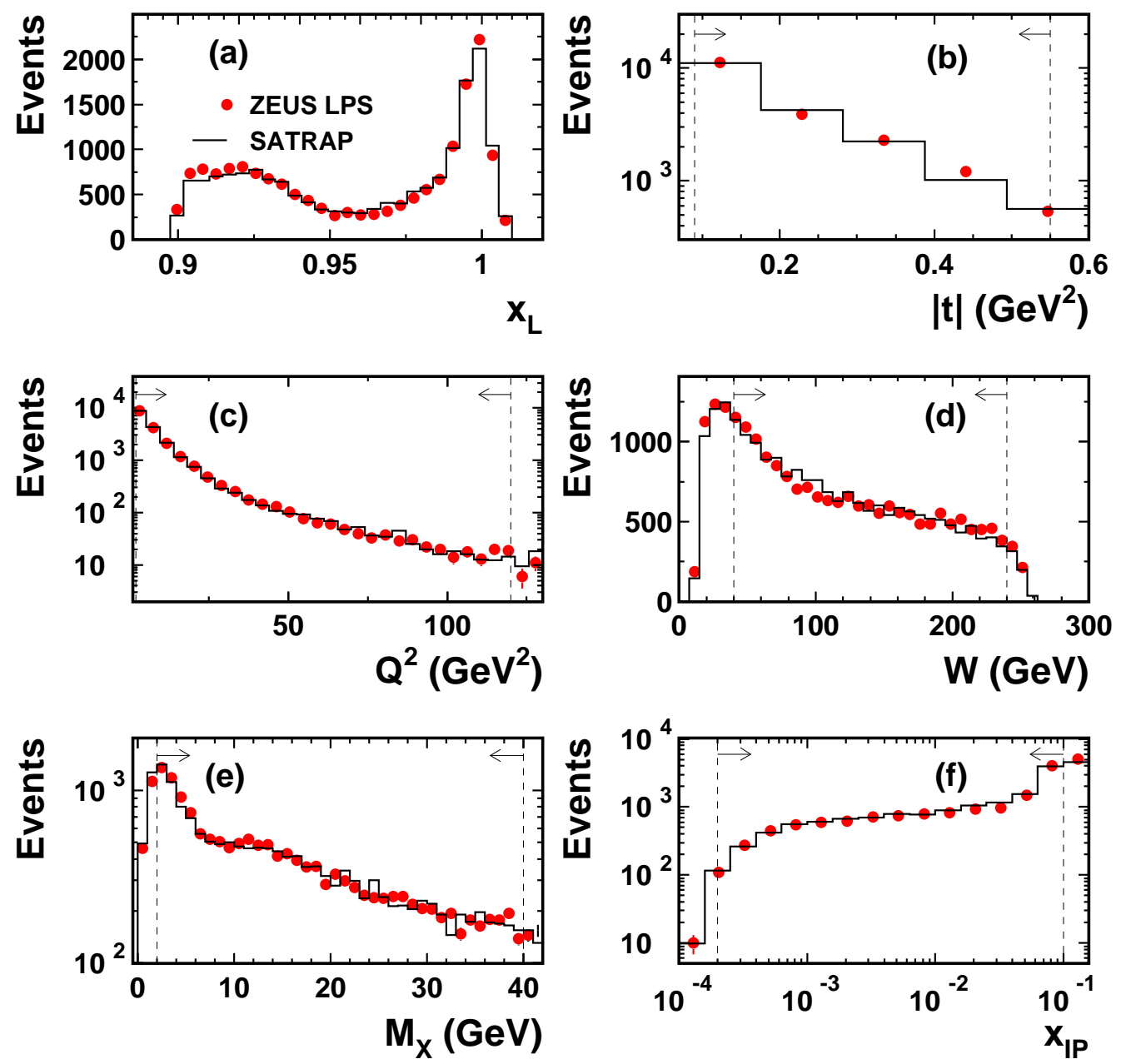

Figure 3: Comparison of the distributions measured (dots) and obtained with the reweighted SATRAP $M C$ (histograms) for (a) $x_{L}$, (b) $|t|$, (c) $Q^{2}$, (d) $W$, (e) $M_{X}$ and $(f) x_{I P}$ in the LPS analysis. Other details as in caption for Fig. 2. 


\section{ZEUS}
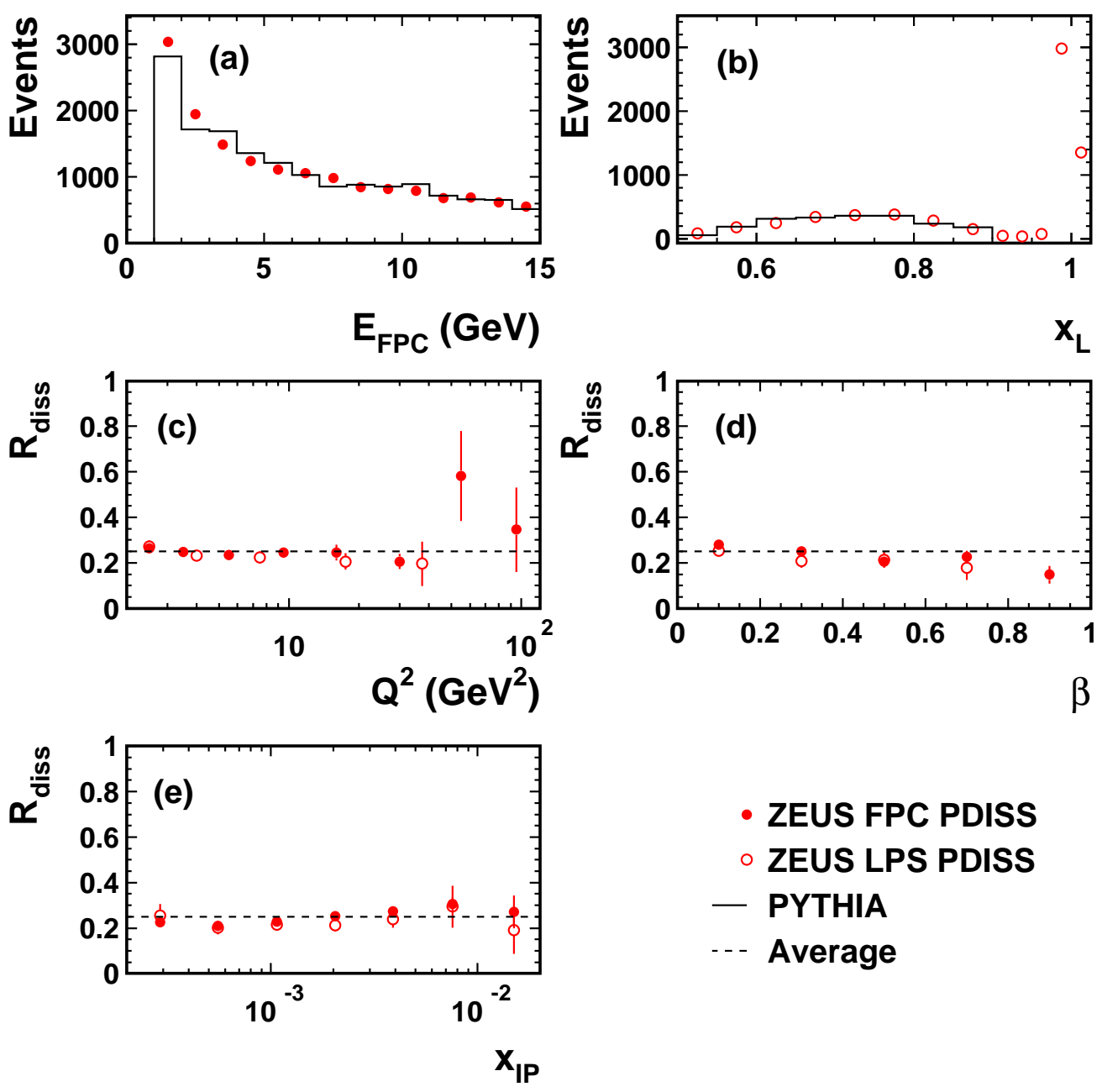

Figure 4: (a) The energy distribution in the FPC, $E_{\mathrm{FPC}}$, for the sample of protondissociative candidate events (FPC PDISS). The data (dots) are compared to the expectation of the reweigthed PYTHIA $M C$ (histogram), normalised to the data. (b) The $x_{L}$ distribution in the LPS for the sample of proton-dissociative candidate events (LPS PDISS). The data (open circles) are compared to the expectation of the reweigthed PYTHIA $M C$ (histogram), normalised to the data in the range $0.5<$ $x_{L}<0.9$. The data points for $x_{L}>0.9$ are also shown for completeness. The extracted fraction of proton-dissociative events, $R_{\text {diss, }}$ from the two samples as a function of (c) $Q^{2},(d) \beta$ and (e) $x_{\mathbb{I P}}$. The dashed lines in (c), (d) and (e) represent the average of the points. 


\section{ZEUS}

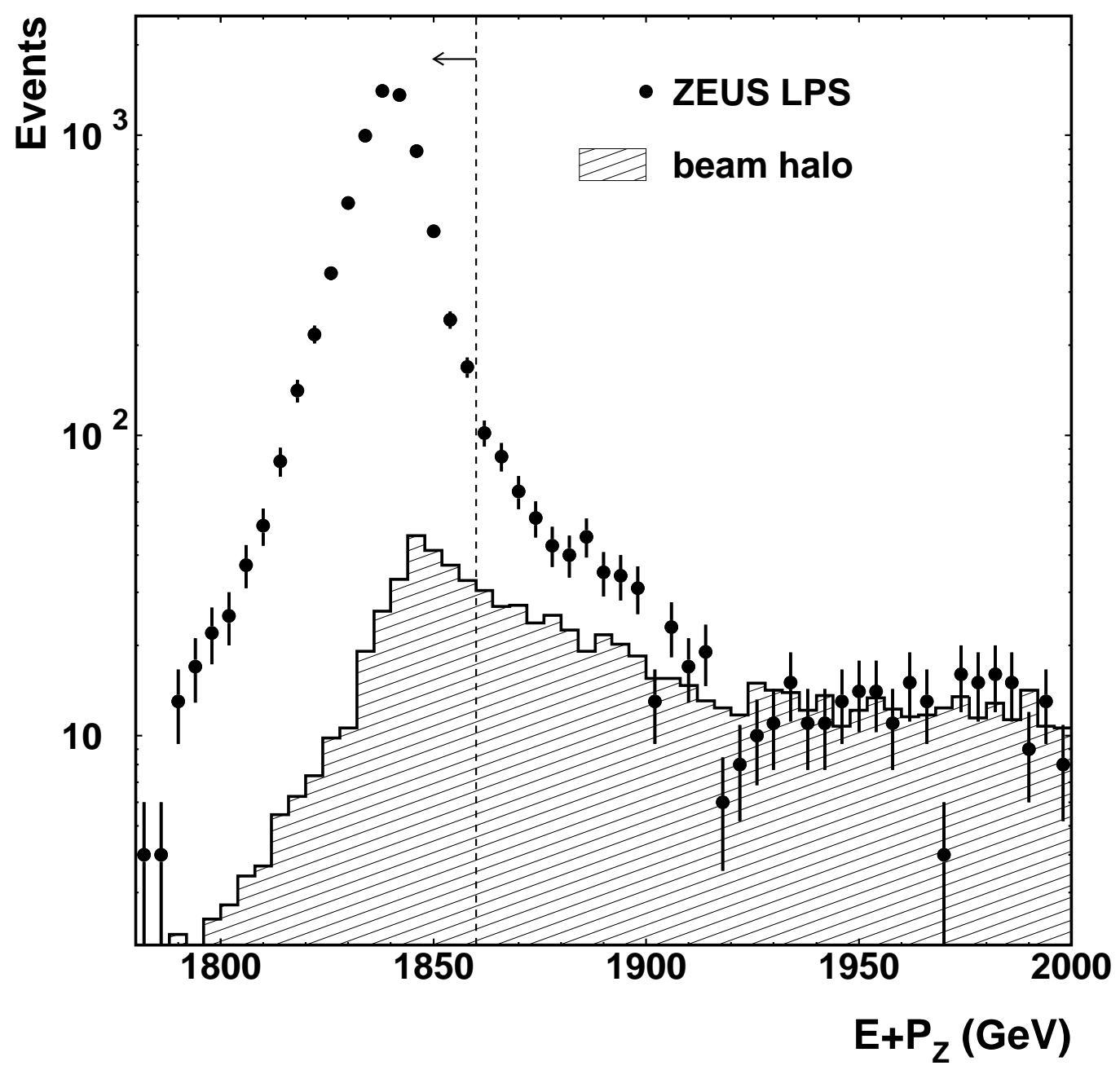

Figure 5: Distribution of $E+P_{Z}$ for the LPS events (dots). The dashed histogram represents the estimate of the beam-halo background normalised for $E+P_{Z}>$ $1925 \mathrm{GeV}$. The vertical dashed line at $E+P_{Z}=1860 \mathrm{GeV}$ represents the selection cut used in the analysis. 
ZEUS
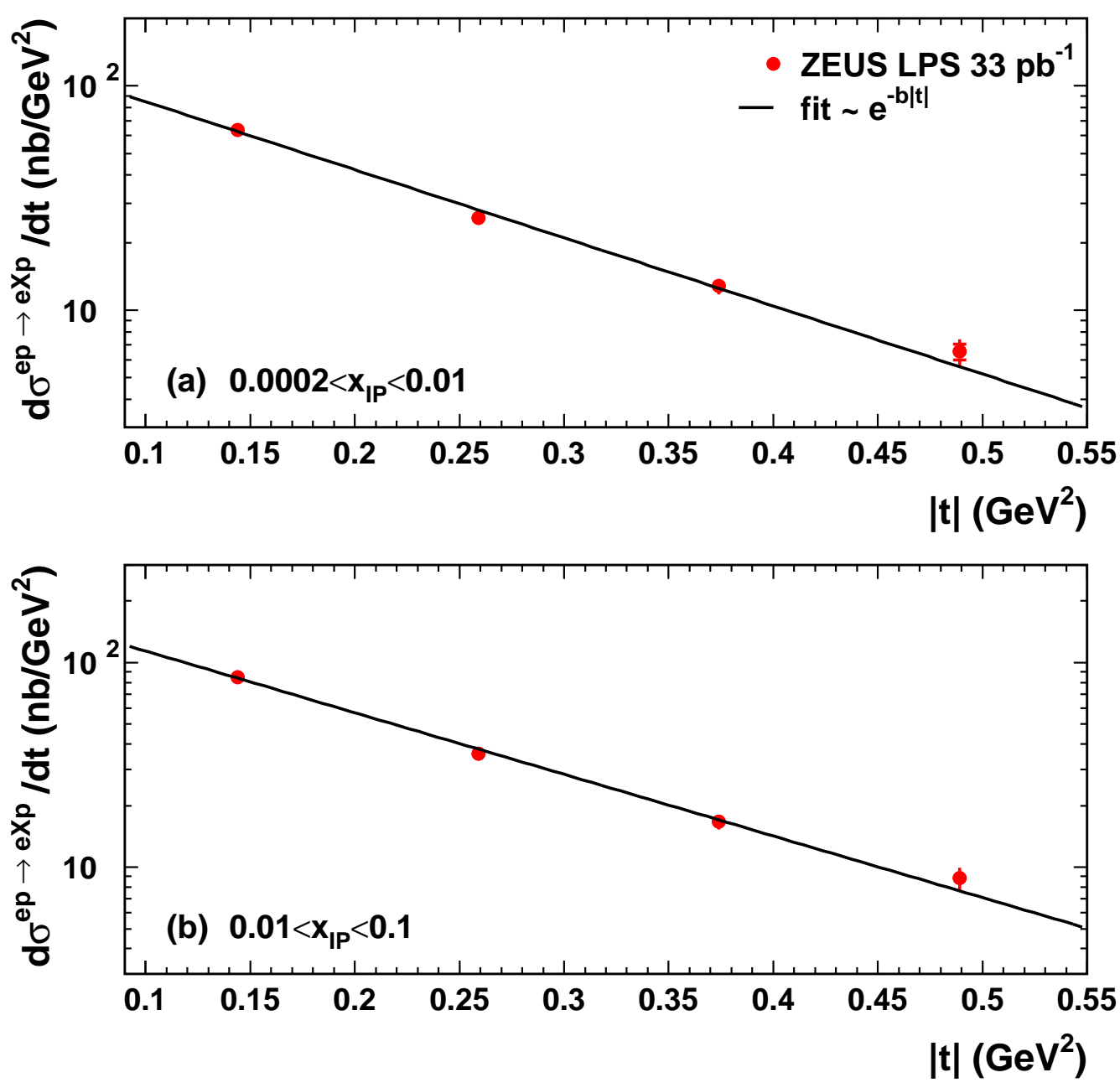

Figure 6: The differential cross-section $d \sigma^{e p \rightarrow e X p} / d t$ for (a) $0.0002<x_{\mathbb{P}}<0.01$ and (b) $0.01<x_{\mathbb{P}}<0.1$. The lines show the results of fits with the function $d \sigma^{e p \rightarrow e X p} / d t \propto e^{-b|t|}$. The inner error bars show the statistical uncertainties and the full bars indicate the statistical and the systematic uncertainties added in quadrature. The normalisation uncertainty of $\pm 7 \%$ is not shown. 


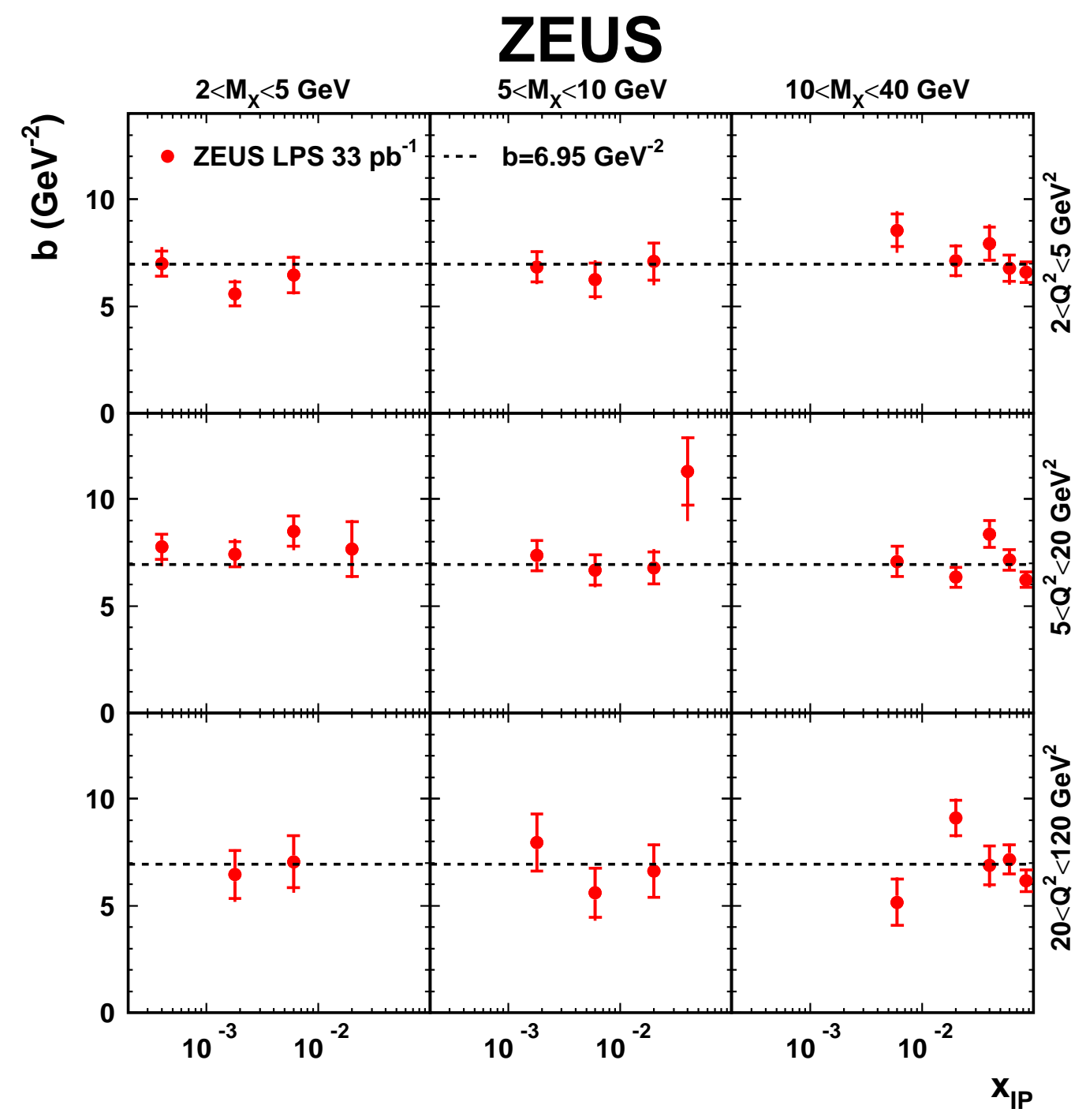

Figure 7: The value of the exponential-slope parameter $b$ of the differential crosssection $d \sigma^{e p \rightarrow e X p} / d t \propto e^{-b|t|}$ as a function of $x_{\mathbb{I P}}$ in bins of $Q^{2}$ and $M_{X}$. The dashed line corresponds to the value of $b=6.95 \mathrm{GeV}^{-2}$, the average $t$-slope over the measured region. The inner error bars show the statistical uncertainties and the full bars indicate the statistical and the systematic uncertainties added in quadrature. 
ZEUS
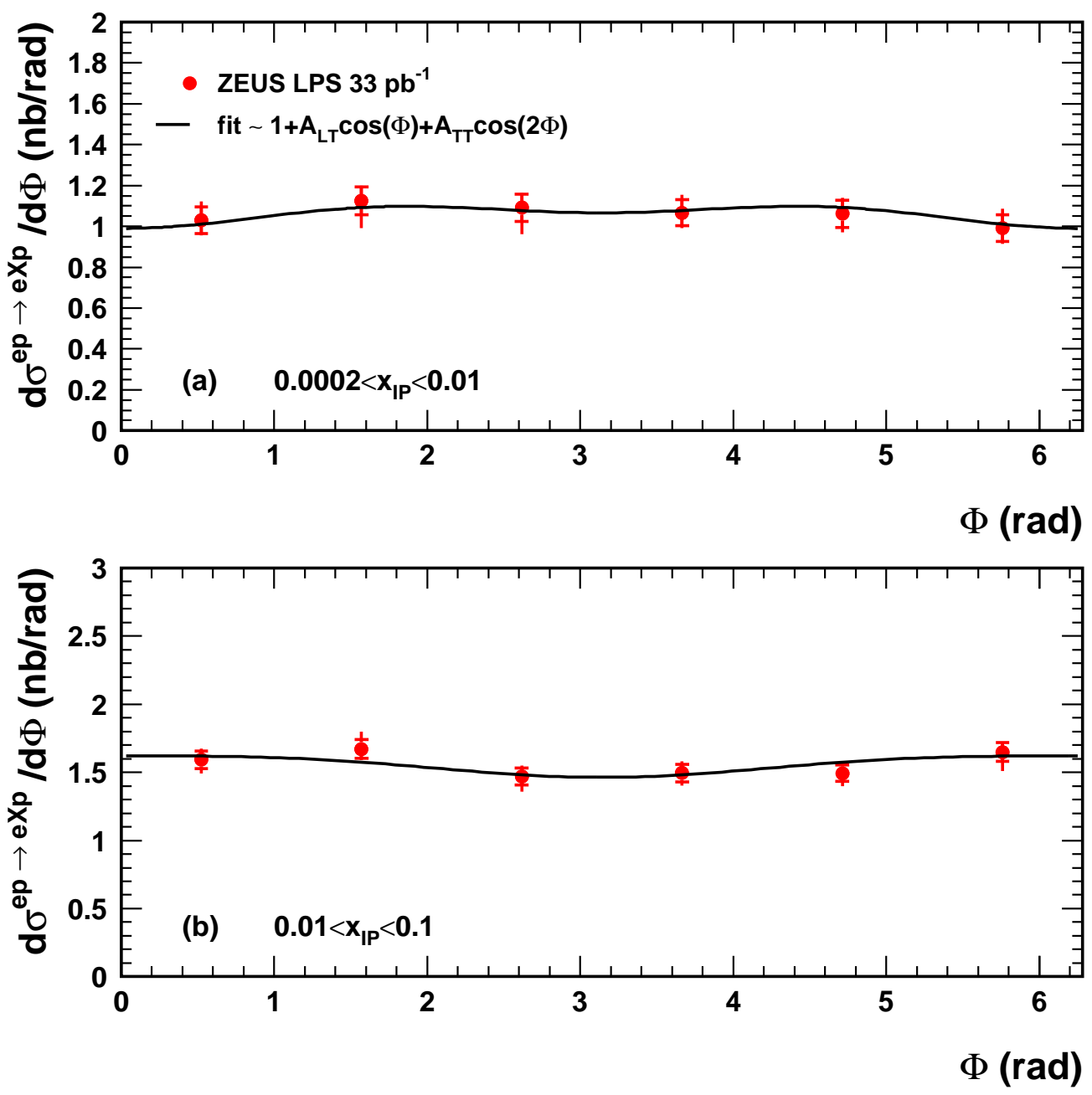

Figure 8: The differential cross-section $d \sigma^{e p \rightarrow e X p} / d \Phi$ in the kinematic ranges (a) $0.0002<x_{\mathbb{P}}<0.01$ and (b) $0.01<x_{\mathbb{P}}<0.1$. The line shows the result of the fit described in Section10.2. The inner error bars show the statistical uncertainties and the full bars indicate the statistical and the systematic uncertainties added in quadrature. The normalisation uncertainty of ${ }_{-7}^{+11} \%$ is not shown. 


\section{ZEUS}
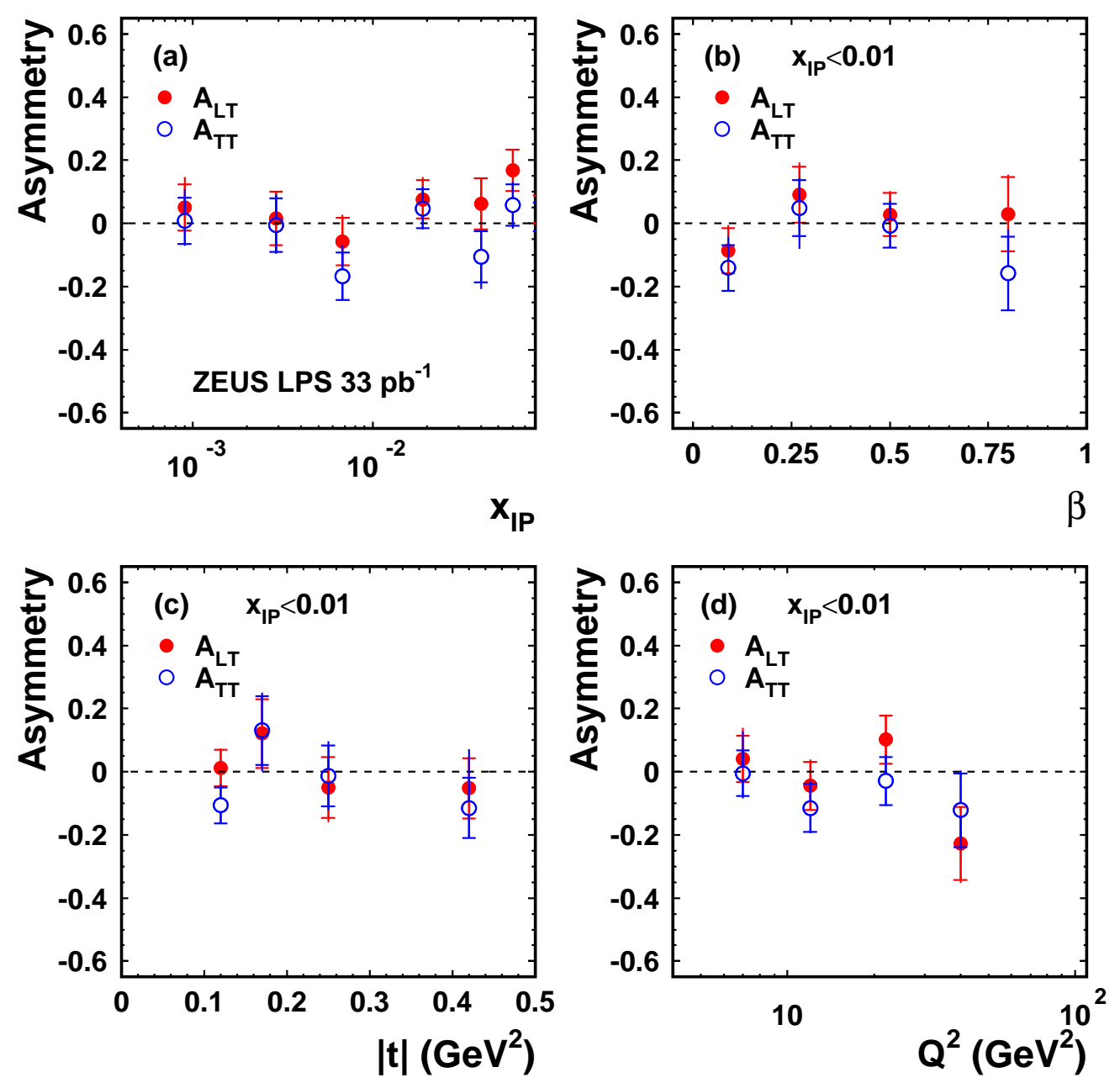

Figure 9: The azimuthal asymmetries $A_{L T}$ (open circles) and $A_{T T}$ (dots) as a function of (a) $x_{\mathbb{P P}}$ and (b) $\beta$, (c) $|t|$ and (d) $Q^{2}$ for $x_{\mathbb{I P}}<0.01$. The inner error bars show the statistical uncertainties and the full bars indicate the statistical and the systematic uncertainties added in quadrature. 


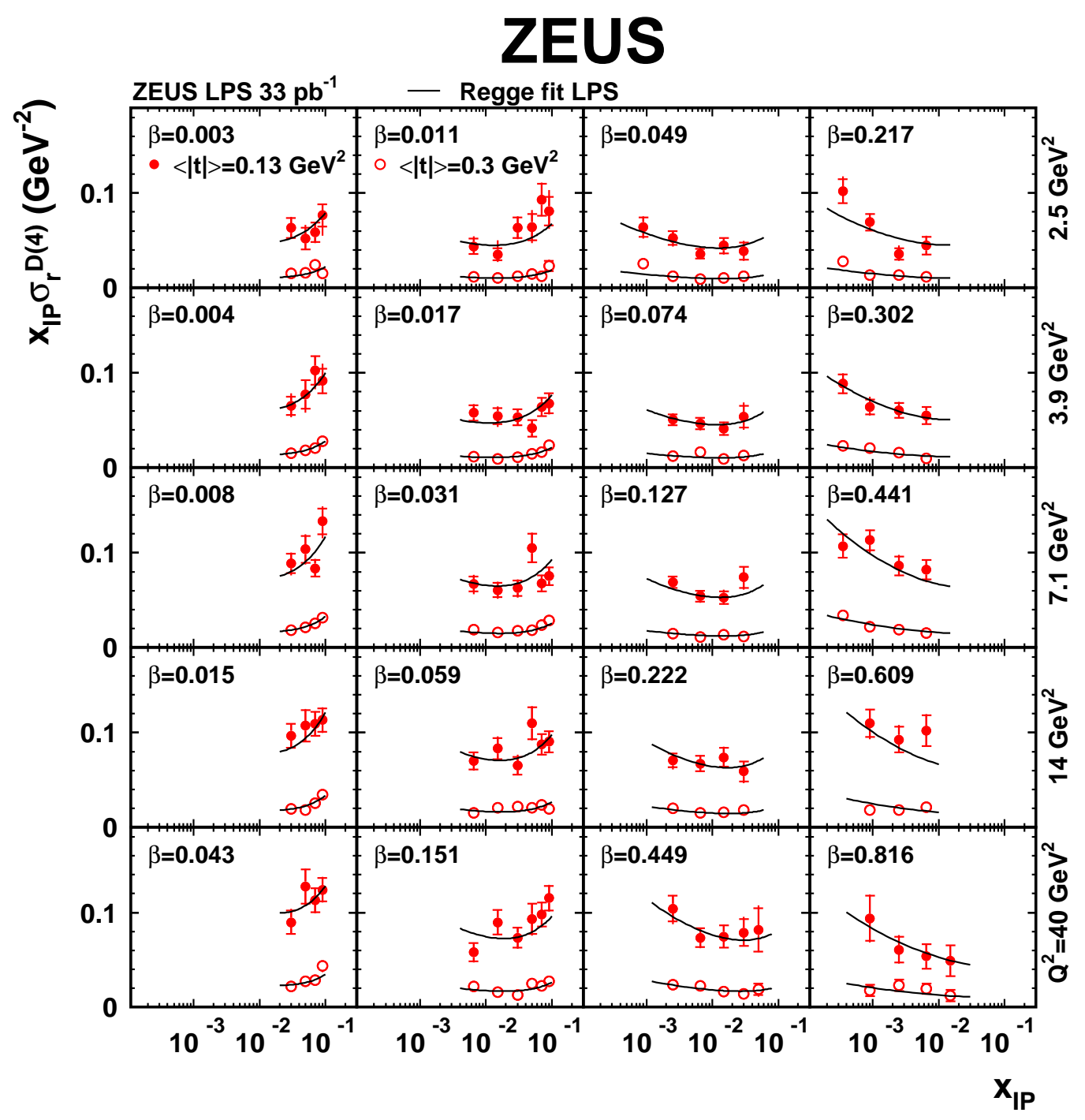

Figure 10: The reduced diffractive cross section multiplied by $x_{\mathbb{P}}, x_{\mathbb{P}} \sigma_{r}^{D(4)}$, obtained with the LPS method in two $t$ bins as a function of $x_{\mathbb{P}}$ for different values of $Q^{2}$ and $\beta$. The lines are the result of the Regge fit described in Section 10.5. The inner error bars show the statistical uncertainties and the full bars indicate the statistical and the systematic uncertainties added in quadrature. The normalisation uncertainty of $\pm 7 \%$ is not shown. 


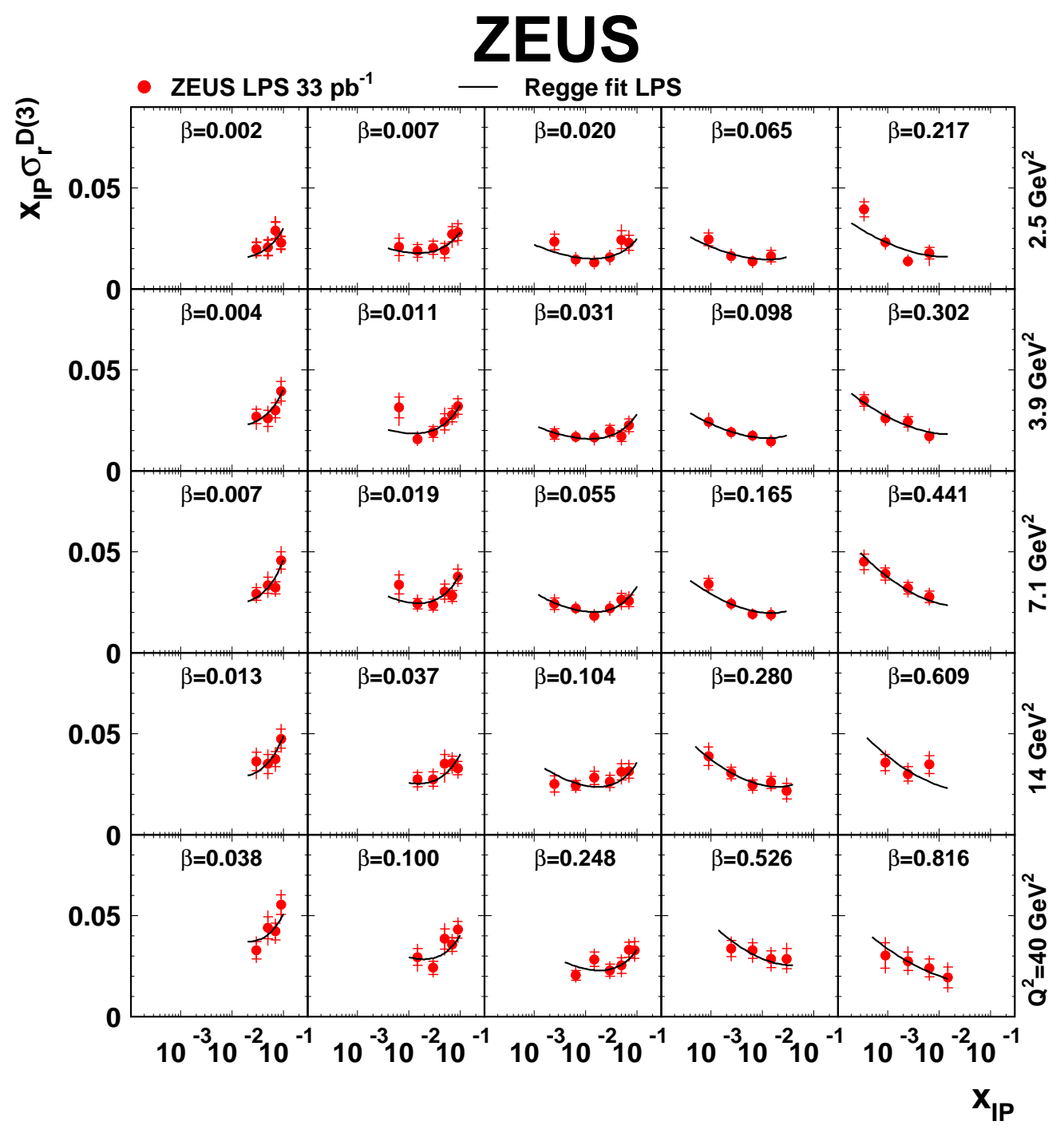

Figure 11: The reduced diffractive cross section multiplied by $x_{\mathbb{P}}, x_{\mathbb{P}} \sigma_{r}^{D(3)}$, obtained with the LPS method as a function of $x_{\mathbb{I P}}$ for different values of $Q^{2}$ and $\beta$. The lines are the result of the Regge fit described in Section 10.5. The inner error bars show the statistical uncertainties and the full bars indicate the statistical and the systematic uncertainties added in quadrature. The normalisation uncertainty of ${ }_{-7}^{+11 \%}$ is not shown. 


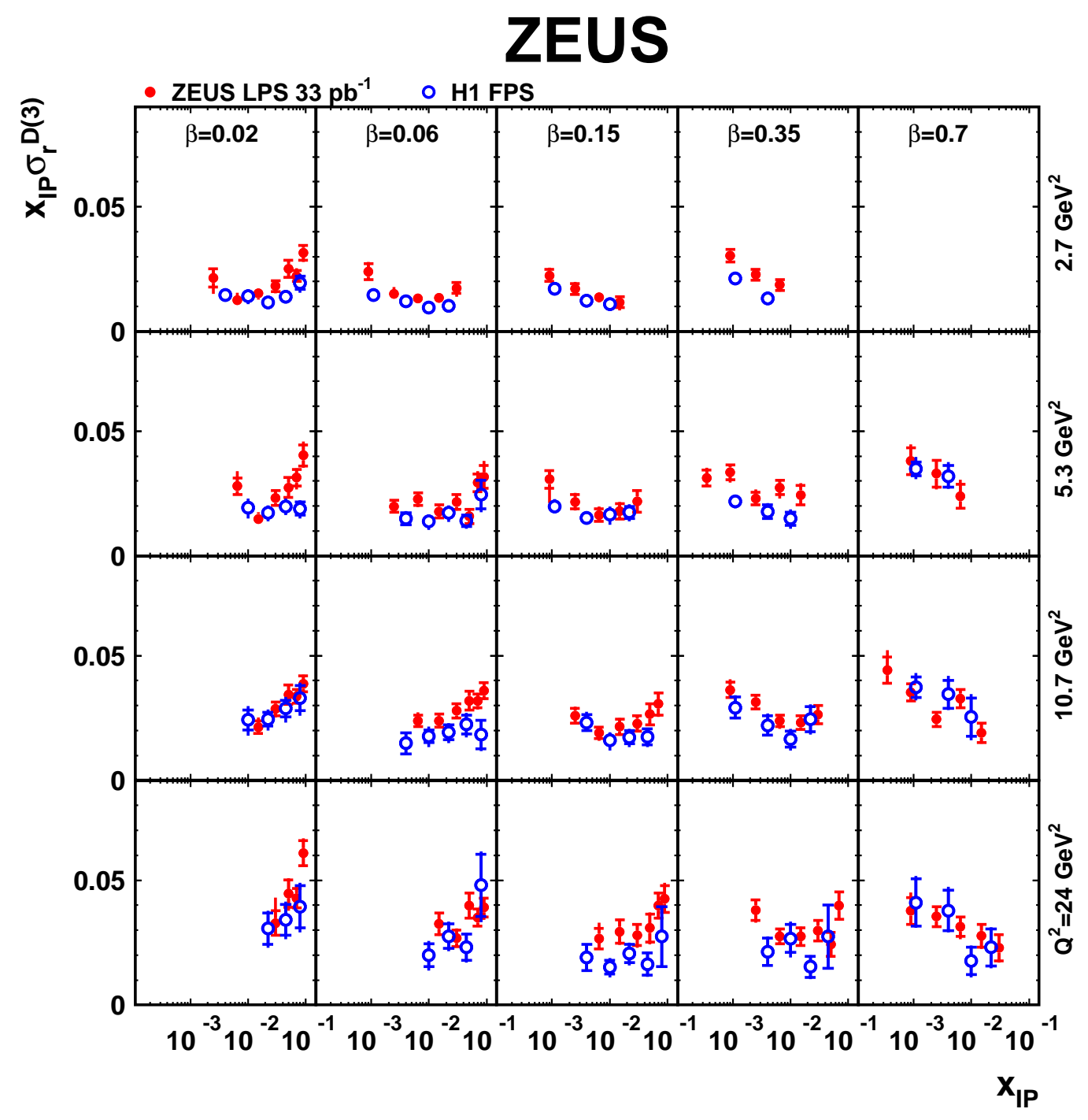

Figure 12: The reduced diffractive cross section multiplied by $x_{\mathbb{P}}, x_{\mathbb{P}} \sigma_{r}^{D(3)}$, obtained with the LPS method (dots) as a function of $x_{\mathbb{I P}}$ for different values of $Q^{2}$ and $\beta$ compared with the results obtained with the H1 Forward Proton Spectrometer (open circles). The inner error bars show the statistical uncertainties and the full bars indicate the statistical and the systematic uncertainties added in quadrature. The normalisation uncertainty of ${ }_{-7}^{+11} \%$ of the ZEUS data is not shown, nor is that of the H1 data ( $\pm 10 \%)$. 


\section{ZEUS}

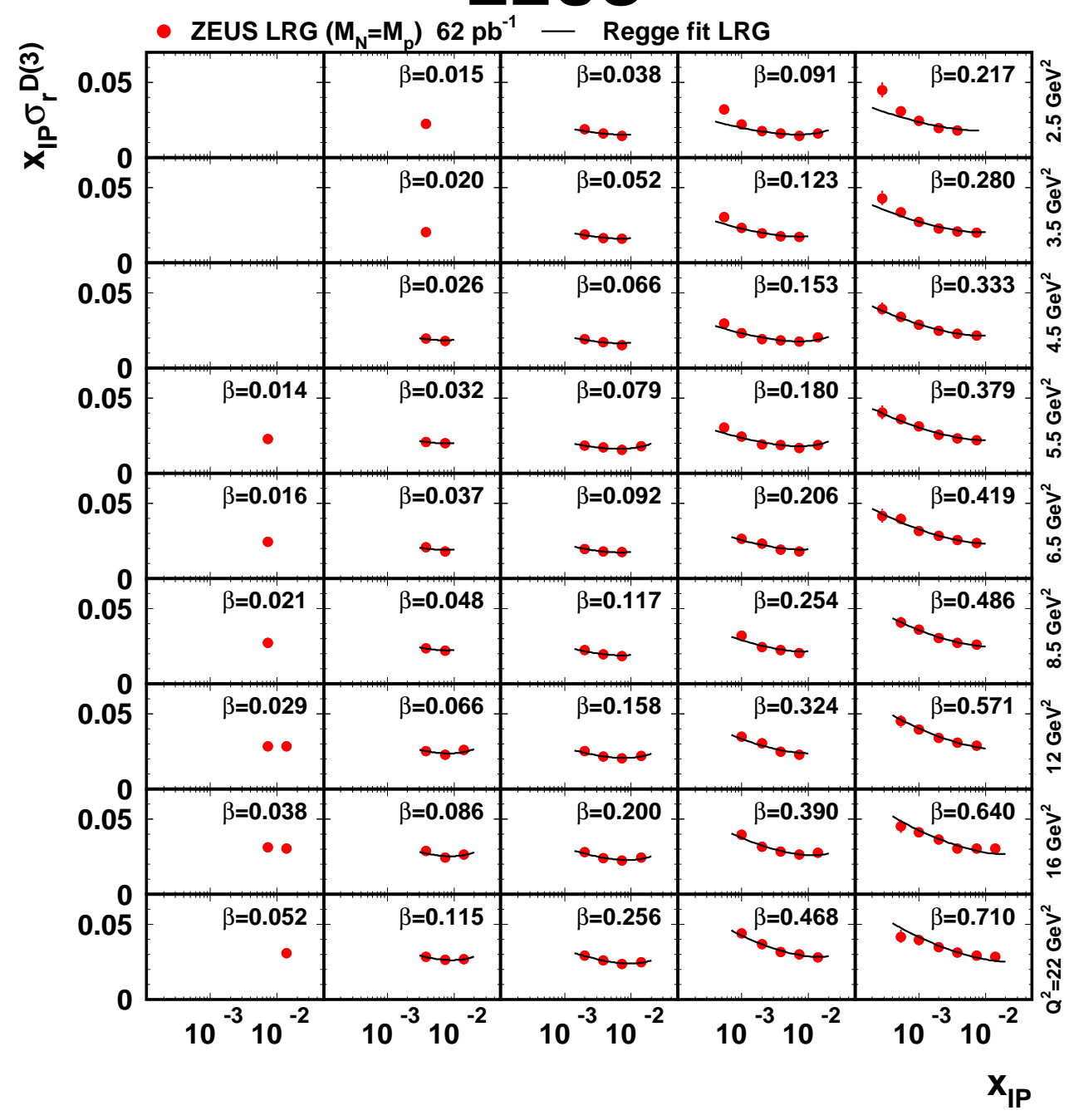

Figure 13: The reduced diffractive cross section multiplied by $x_{\mathbb{P}}, x_{\mathbb{P}} \sigma_{r}^{D(3)}$, obtained with the LRG method as a function of $x_{\mathbb{P}}$ for different values of $Q^{2}$ and $\beta$ at low $Q^{2}$ values. The lines are the result of the Regge fit described in Section 10.5. The inner error bars show the statistical uncertainties and the full bars indicate the statistical and the systematic uncertainties added in quadrature. The data are corrected for the proton-dissociative background to $M_{N}=M_{p}$ as described in Section 8.1. The normalisation uncertainty of $\pm 5 \%$ is not shown. 


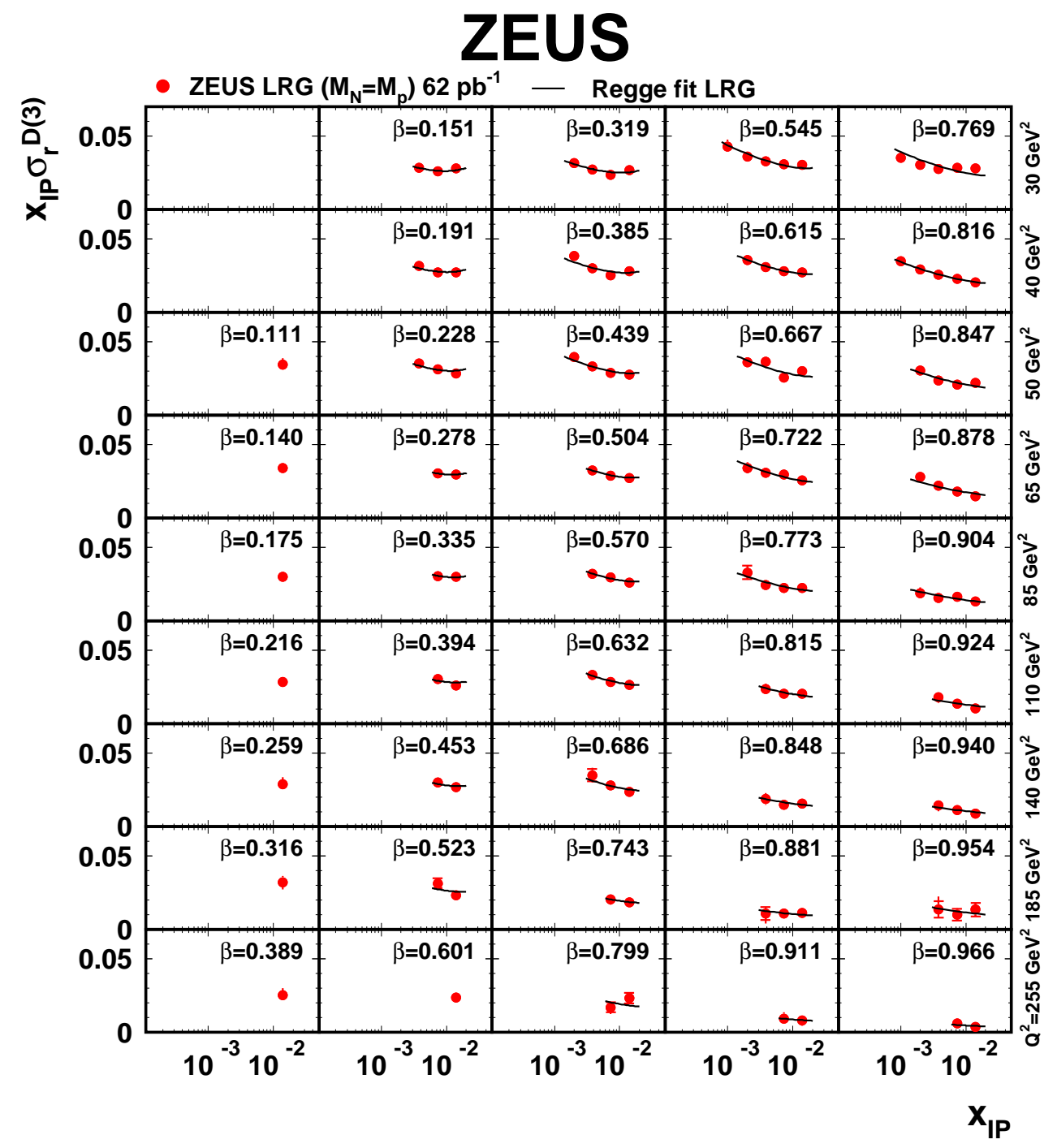

Figure 14: The reduced diffractive cross section multiplied by $x_{\mathbb{I P}}, x_{\mathbb{P}} \sigma_{r}^{D(3)}$, obtained with the LRG method as a function of $x_{\mathbb{P}}$ for different values of $Q^{2}$ and $\beta$ at high $Q^{2}$ values. Other details as in caption for Fig. 13. 


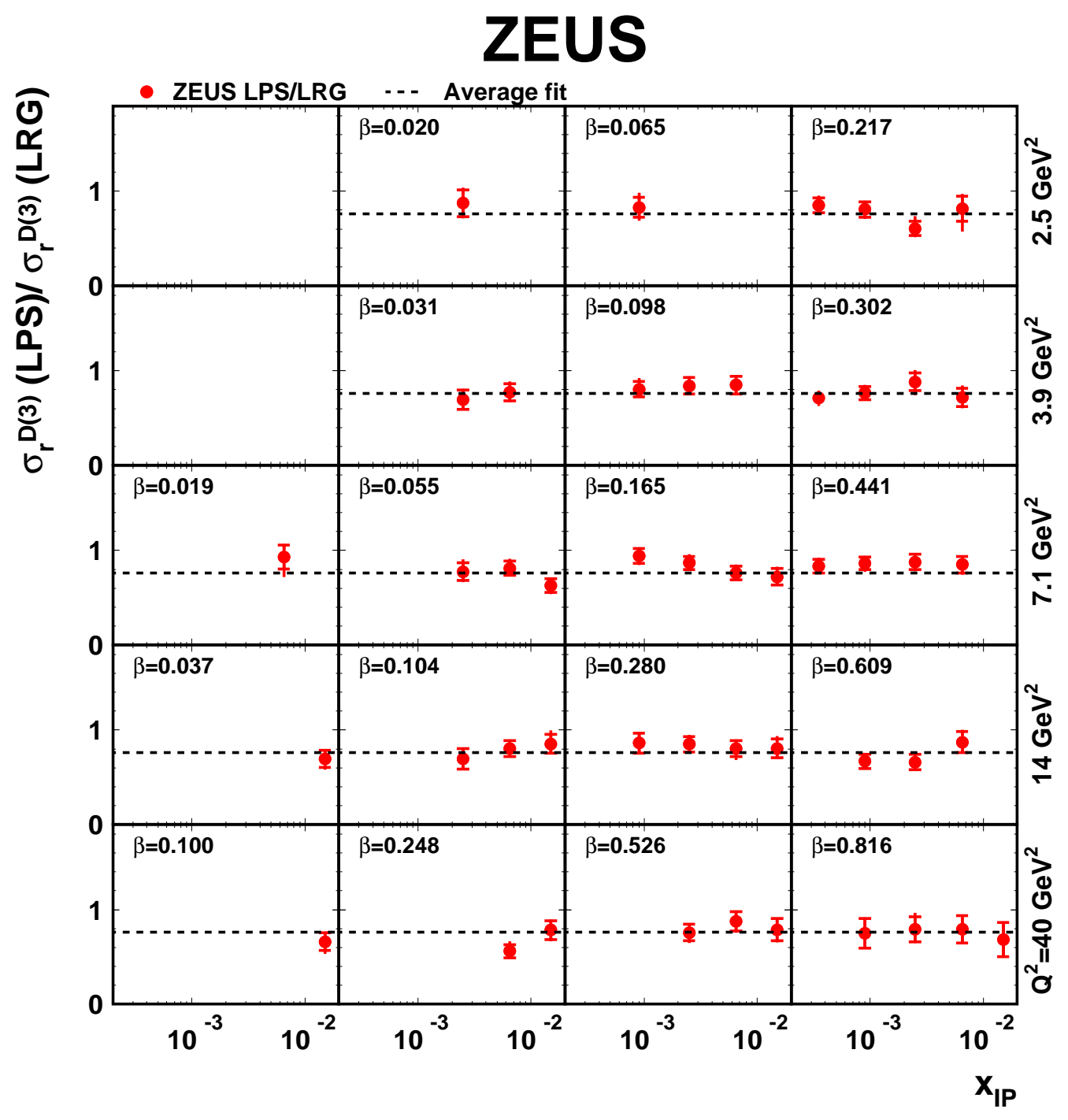

Figure 15: The ratio of the reduced diffractive cross sections, $\sigma_{r}^{D(3)}$, as obtained with the LPS and the LRG methods, before the subtraction of the proton-dissociative background, as a function of $x_{\mathbb{P}}$ for different values of $Q^{2}$ and $\beta$. The lines indicate the average value of the ratio. The inner error bars show the statistical uncertainties and the full bars indicate the statistical and the systematic uncertainties added in quadrature. 


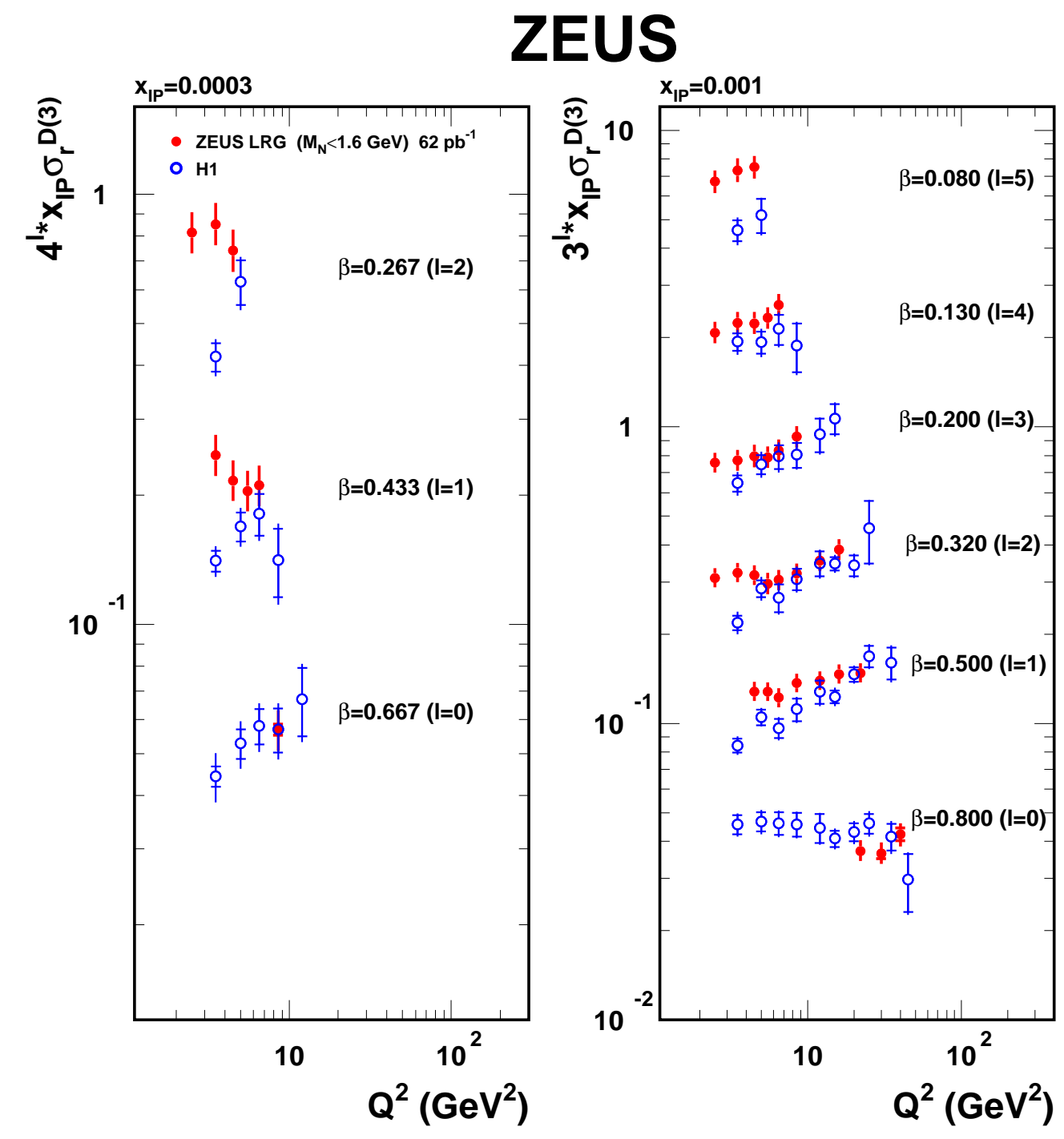

Figure 16: The reduced diffractive cross section multiplied by $x_{\mathbb{I P}}, x_{\mathbb{P}} \sigma_{r}^{D(3)}$, obtained with the LRG method (dots) at $x_{\mathbb{P}}=0.0003$ and $x_{\mathbb{P}}=0.001$ as a function of $Q^{2}$ for different $\beta$ values compared with the $H 1$ results (open circles), also obtained with the LRG method. The inner error bars show the statistical uncertainties and the full bars indicate the statistical and the systematic uncertainties added in quadrature. The ZEUS data are corrected to $M_{N}<1.6 \mathrm{GeV}$ as described in Section 10.4. The $8 \%$ uncertainty on the correction is not shown, nor is the $7 \%$ relative normalisation uncertainty between the ZEUS and H1 data sets. 


\section{ZEUS}
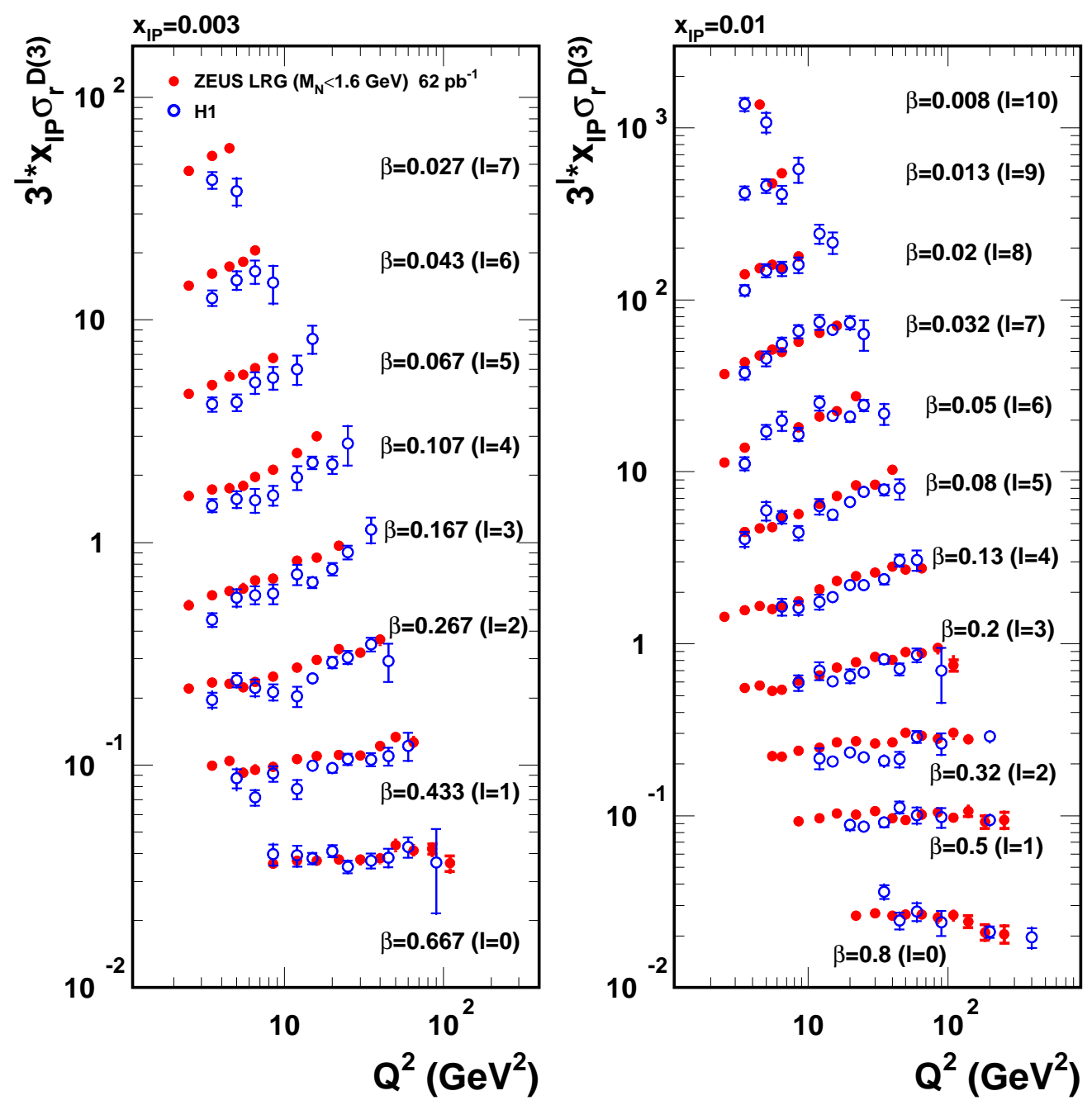

Figure 17: The reduced diffractive cross section multiplied by $x_{\mathbb{P}}, x_{\mathbb{P}} \sigma_{r}^{D(3)}$, obtained with the LRG method (dots) at $x_{\mathbb{I P}}=0.003$ and $x_{\mathbb{P}}=0.01$ as a function of $Q^{2}$ for different $\beta$ values compared with the $H 1$ results (open circles), also obtained with the LRG method. Other details as in caption for Fig. 16. 


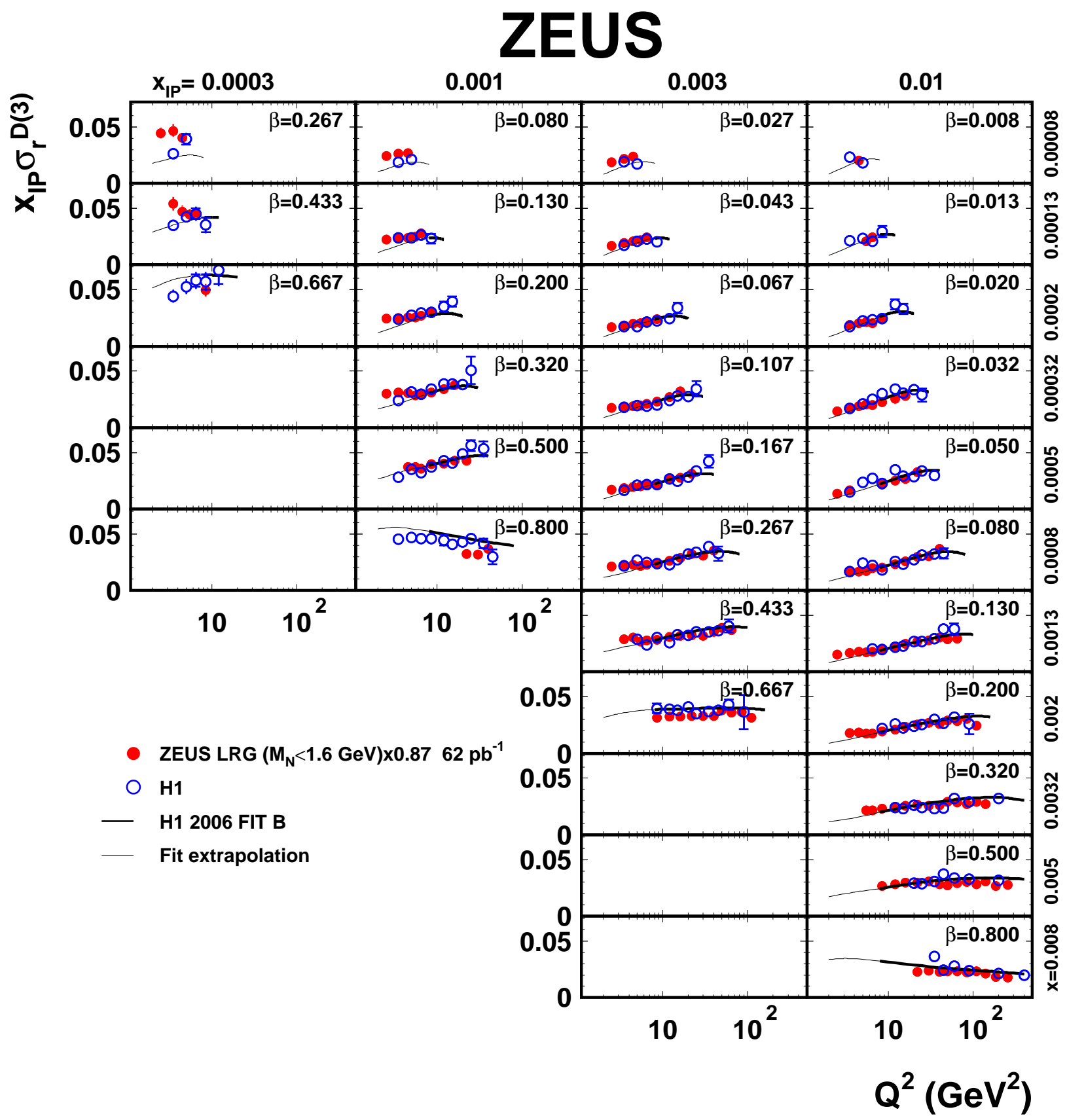

Figure 18: The reduced diffractive cross section multiplied by $x_{\mathbb{P}}, x_{\mathbb{P}} \sigma_{r}^{D(3)}, o b$ tained with the LRG method (dots) as a function of $Q^{2}$ for different $\beta$ and $x_{\mathbb{P}}$ values compared with the $H 1$ results (open circles), also obtained with the LRG method. The lines represent the expectation based on the diffractive parton distribution functions "H1 2006 fit B". The inner error bars show the statistical uncertainties and the full bars indicate the statistical and the systematic uncertainties added in quadrature. The ZEUS data are normalised to the H1 data. 


\section{ZEUS}

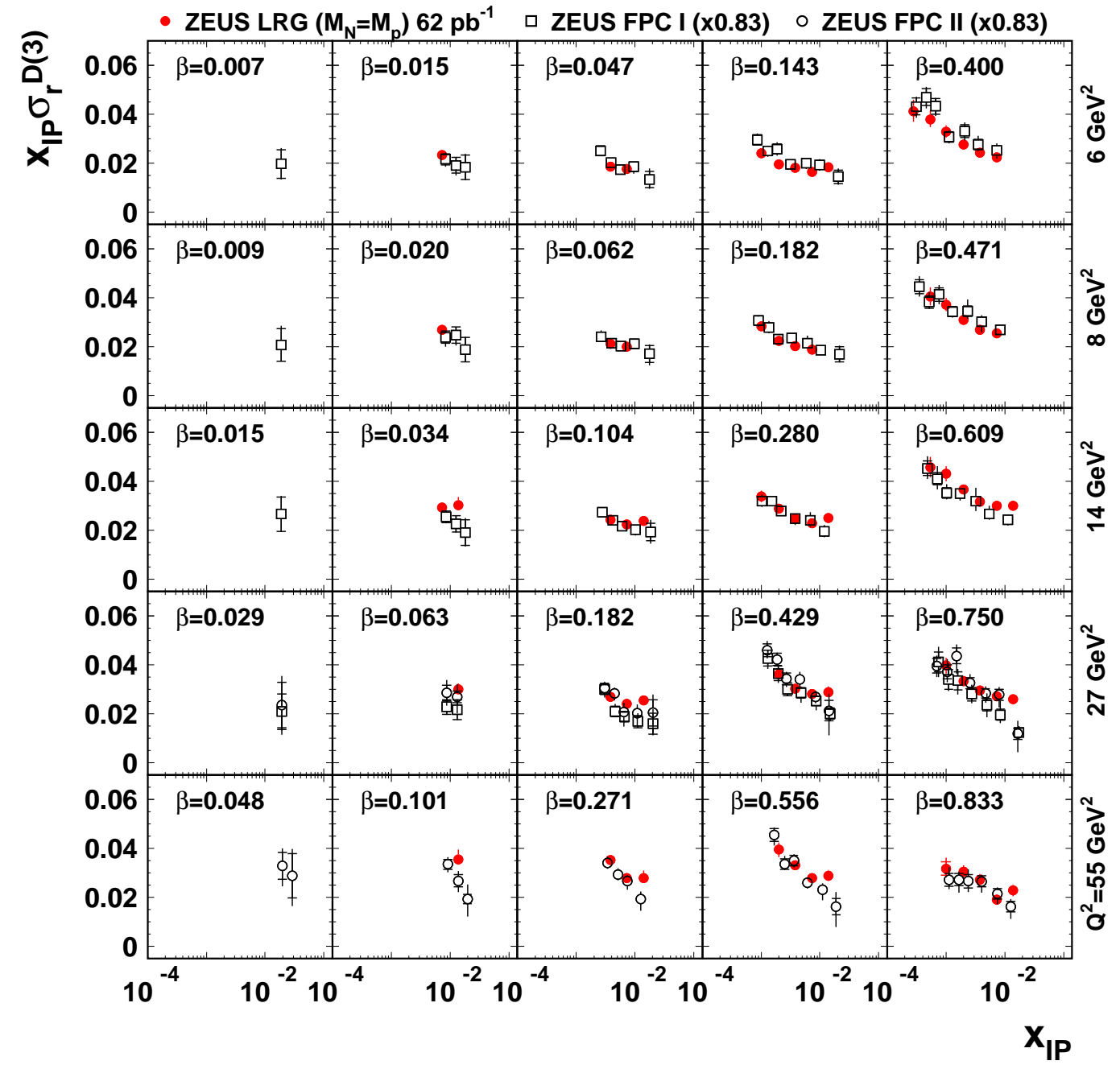

Figure 19: The reduced diffractive cross section multiplied by $x_{\mathbb{P}}, x_{\mathbb{P}} \sigma_{r}^{D(3)}$, obtained with the LRG method (dots) as a function of $x_{\mathbb{P}}$ for different values of $Q^{2}$ and $\beta$ at low $Q^{2}$, compared with the results obtained with the $M_{X}$ method, FPC I (open squares) and FPC II (open circles), scaled by the factor 0.83 described in Section 10.4. The inner error bars show the statistical uncertainties and the full bars indicate the statistical and the systematic uncertainties added in quadrature. 


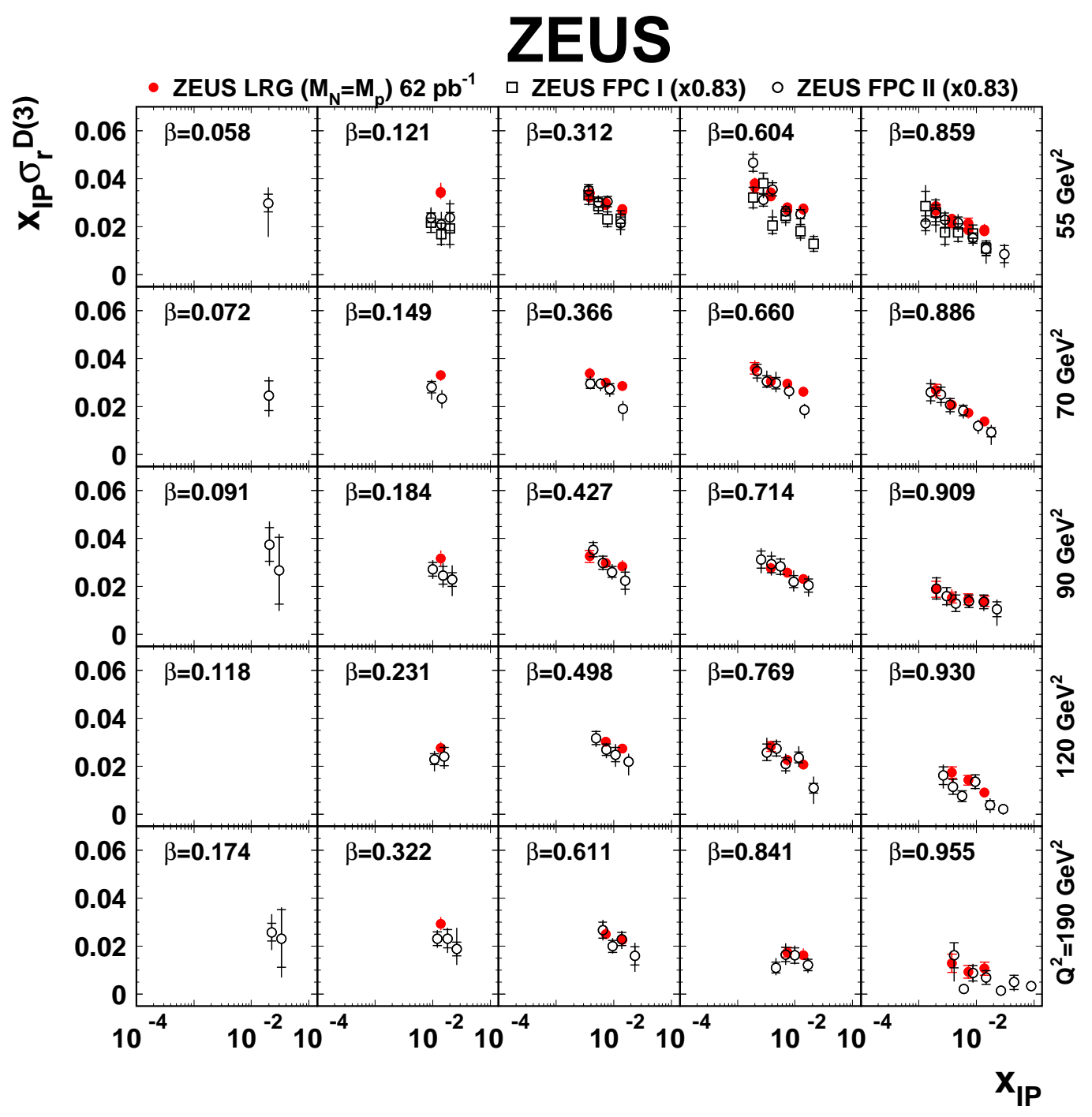

Figure 20: The reduced diffractive cross section multiplied by $x_{\mathbb{P}}, x_{\mathbb{P}} \sigma_{r}^{D(3)}$, obtained with the LRG method (dots) as a function of $x_{\mathbb{I P}}$ for different values of $Q^{2}$ and $\beta$ at high $Q^{2}$. Other details as in caption for Fig. 19. 


\section{ZEUS}

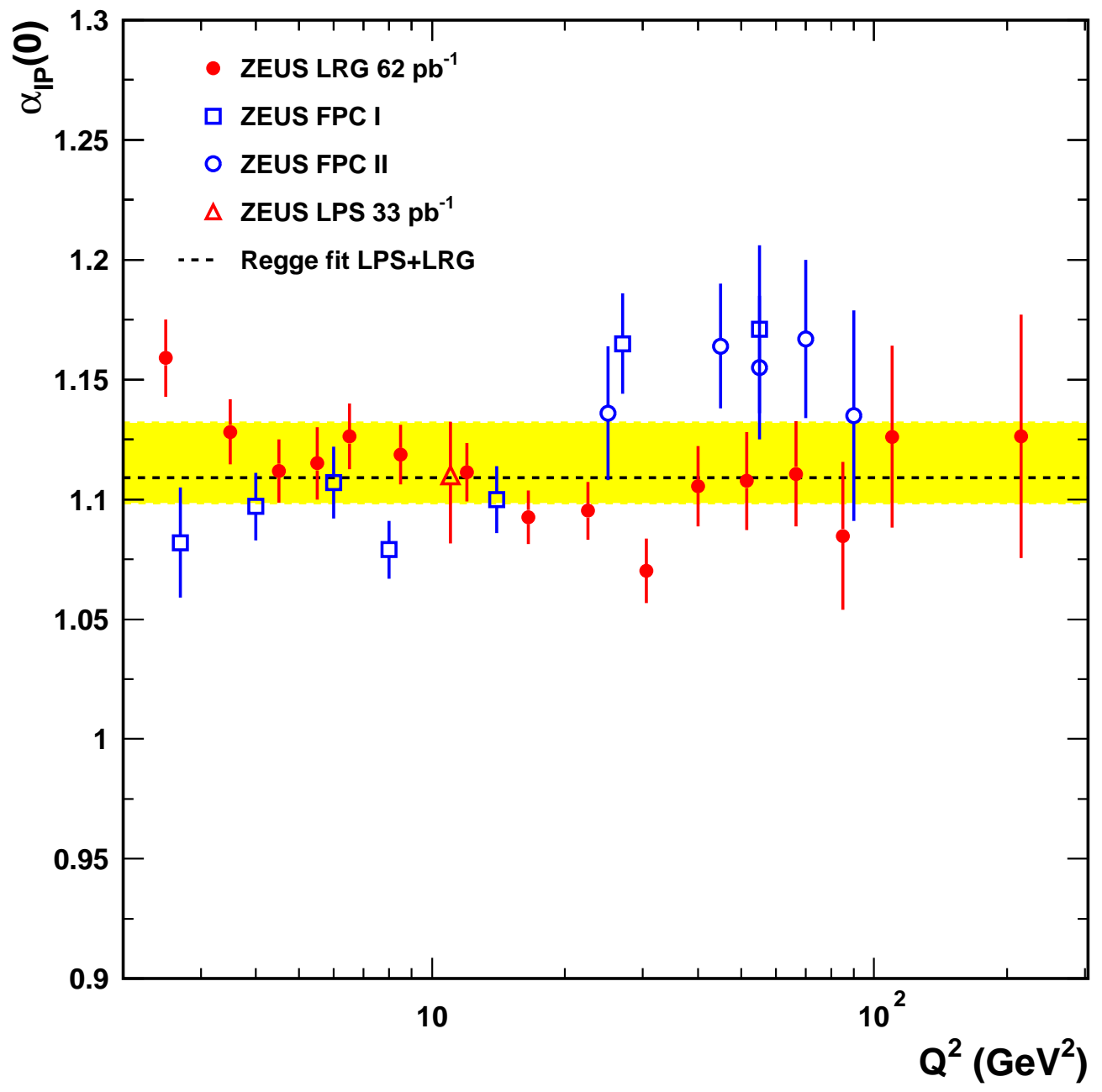

Figure 21: The Pomeron intercept $\alpha_{\mathbb{P}}(0)$ as a function of $Q^{2}$ as obtained from the $L R G$ (dots) and the LPS data (triangles). Also shown are the $M_{X}$-method results, FPC I (open squares) and FPC II (open circles). The error bars indicate the uncertainty from the fit for the $L R G$ and FPC points; they indicate the statistical and systematic uncertainties summed in quadrature for the LPS points. The dashed line indicates the results of the Regge fit to the LPS and LRG data together described in Section 10.5, and the band indicates the size of the total error. 